Western University

Scholarship@Western

Surgery Publications

Surgery Department

2006

\title{
Cyclosporin versus Tacrolimus for Liver Transplanted Patients
}

Elizabeth Haddad

University of Western Ontario, ehaddad2@uwo.ca

Vivian McAlister

University of Western Ontario, vmcalist@uwo.ca

Elizabeth Renouf

University of Western Ontario, erenouf@uwo.ca

Richard Malthaner

University of Western Ontario, richard.malthaner@lhsc.on.ca

Mette S. Kjaer

Rigshospitalet, Copenhagen, Denmark

See next page for additional authors

Follow this and additional works at: https://ir.lib.uwo.ca/surgerypub

Part of the Epidemiology Commons, Oncology Commons, Statistics and Probability Commons, and the Surgery Commons

Citation of this paper:

Haddad, Elizabeth; McAlister, Vivian; Renouf, Elizabeth; Malthaner, Richard; Kjaer, Mette S.; and Gluud, Lise Lotte, "Cyclosporin versus Tacrolimus for Liver Transplanted Patients" (2006). Surgery Publications. 29.

https://ir.lib.uwo.ca/surgerypub/29 
Authors

Elizabeth Haddad, Vivian McAlister, Elizabeth Renouf, Richard Malthaner, Mette S. Kjaer, and Lise Lotte Gluud 


\title{
Cyclosporin versus tacrolimus for liver transplanted patients (Review)
}

\author{
Haddad E, McAlister V, Renouf E, Malthaner R, Kjaer MS, Gluud LL
}

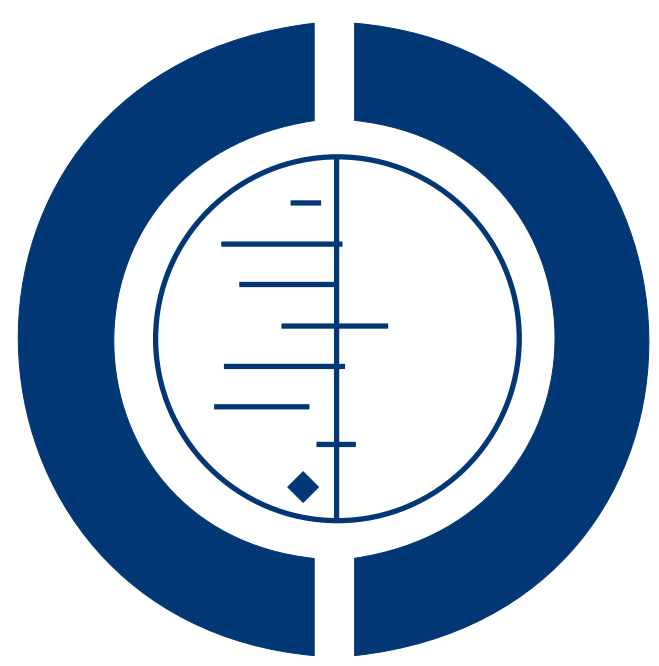

\section{THE COCHRANE COLLABORATION $^{\circledR}$}

This is a reprint of a Cochrane review, prepared and maintained by The Cochrane Collaboration and published in The Cochrane Library 2009, Issue 1

http://www.thecochranelibrary.com

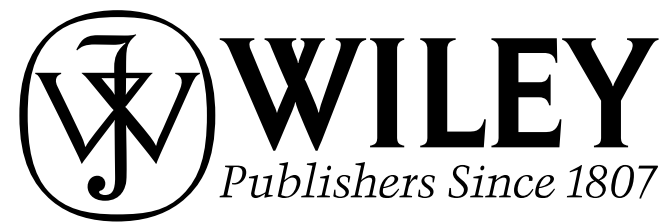

Cyclosporin versus tacrolimus for liver transplanted patients (Review)

Copyright (C) 2009 The Cochrane Collaboration. Published by John Wiley \& Sons, Ltd. 
TABLE OF CONTENTS

HEADER . . . . . . . . . . . . . . . . . . . . . . . . . . . . . . . . 1

ABSTRACT .. . . . . . . . . . . . . . . . . . . . . . . . . . . . . . . . . . . . . . . . . . . . . . . . .

PLAIN LANGUAGE SUMMARY . . . . . . . . . . . . . . . . . . . . . . . . . . . . . . . . . . . . . 2

BACKGROUND . . . . . . . . . . . . . . . . . . . . . . . . . . . . . . . . . . . . . 2

OBJECTIVES . . . . . . . . . . . . . . . . . . . . . . . . . . . . . . . . . . . . . . . 3

METHODS . . . . . . . . . . . . . . . . . . . . . . . . . . . . . . . . . . . . . .

RESULTS . . . . . . . . . . . . . . . . . . . . . . . . . . . . . . . . . . . . . . . . . . 4

DISCUSSION . . . . . . . . . . . . . . . . . . . . . . . . . . . . . . . . . . . . . 6

AUTHORS' CONCLUSIONS . . . . . . . . . . . . . . . . . . . . . . . . . . . . . . . . . . . . . . . . . 7

ACKNOWLEDGEMENTS . . . . . . . . . . . . . . . . . . . . . . . . . . . . . . . . 7

REFERENCES . . . . . . . . . . . . . . . . . . . . . . . . . . . . . . . . . . . . . . . . . . . . 7

CHARACTERISTICS OF STUDIES . . . . . . . . . . . . . . . . . . . . . . . . . . . . . . . . . . . . . . . . 9

DATA AND ANALYSES . . . . . . . . . . . . . . . . . . . . . . . . . . . . . . . . . . 30

Analysis 1.1. Comparison 1 Cyclosporin versus tacrolimus, Outcome 1 Mortality. . . . . . . . . . . . . . . . 36

Analysis 1.2. Comparison 1 Cyclosporin versus tacrolimus, Outcome 2 Graft loss. . . . . . . . . . . . . . . . . 37

Analysis 1.3. Comparison 1 Cyclosporin versus tacrolimus, Outcome 3 Acute rejection. . . . . . . . . . . . . 38

Analysis 1.4. Comparison 1 Cyclosporin versus tacrolimus, Outcome 4 Steroid-resistent rejection. . . . . . . . . . 39

Analysis 1.5. Comparison 1 Cyclosporin versus tacrolimus, Outcome 5 Dialysis (de-novo requirement posttransplantation). . . . . . . . . . . . . . . . . . . . . . . . . . . . . . . . . . 40

Analysis 1.6. Comparison 1 Cyclosporin versus tacrolimus, Outcome 6 Creatinine (umol/L) before transplantation. . 40

Analysis 1.7. Comparison 1 Cyclosporin versus tacrolimus, Outcome 7 Creatinine (umol/L) 12 months after transplantation. . . . . . . . . . . . . . . . . . . . . . . . . . . . . . . . .

Analysis 1.8. Comparison 1 Cyclosporin versus tacrolimus, Outcome 8 Diabetes mellitus: initially diagnosed after transplantation. . . . . . . . . . . . . . . . . . . . . . . . . . . . . . . . . .

Analysis 1.9. Comparison 1 Cyclosporin versus tacrolimus, Outcome 9 Post transplant lymphoproliferative disease.

Analysis 1.10. Comparison 1 Cyclosporin versus tacrolimus, Outcome 10 Patients withdrawn from tacrolimus or cyclosporin. . . . . . . . . . . . . . . . . . . . . . . . . . . . . . . . . . . 43

Analysis 2.1. Comparison 2 Stratified analysis, by cyclosporin formulation, Outcome 1 Mortality. . . . . . . . . 44

Analysis 2.2. Comparison 2 Stratified analysis, by cyclosporin formulation, Outcome 2 Graft loss. . . . . . . . . . 45

Analysis 2.3. Comparison 2 Stratified analysis, by cyclosporin formulation, Outcome 3 Acute rejection. . . . . . . 46

Analysis 2.4. Comparison 2 Stratified analysis, by cyclosporin formulation, Outcome 4 Steroid-resistent rejection. $\quad . \quad 47$

Analysis 2.5. Comparison 2 Stratified analysis, by cyclosporin formulation, Outcome 5 Dialysis (de-novo requirement posttransplantation). . . . . . . . . . . . . . . . . . . . . . . . . . . . . . . . . . 48

Analysis 2.6. Comparison 2 Stratified analysis, by cyclosporin formulation, Outcome 6 Diabetes mellitus: initially diagnosed after transplantation.

Analysis 2.7. Comparison 2 Stratified analysis, by cyclosporin formulation, Outcome 7 Post transplant lymphoproliferative disease. . . . . . . . . . . . . . . . . . . . . . . . . . . . . . . . . . . . .

Analysis 2.8. Comparison 2 Stratified analysis, by cyclosporin formulation, Outcome 8 Patients withdrawn from tacrolimus or cyclosporin.

Analysis 3.1. Comparison 3 Stratified analysis, by inclusion of children, Outcome 1 Mortality. . . . . . . . . . .

Analysis 3.2. Comparison 3 Stratified analysis, by inclusion of children, Outcome 2 Graft loss. . . . . . . . . . . . $\quad . \quad 53$

Analysis 3.3. Comparison 3 Stratified analysis, by inclusion of children, Outcome 3 Acute rejection. . . . . . . . . 54

Analysis 3.4. Comparison 3 Stratified analysis, by inclusion of children, Outcome 4 Steroid-resistent rejection. $\quad$. $\quad 55$

Analysis 3.5. Comparison 3 Stratified analysis, by inclusion of children, Outcome 5 Dialysis (de-novo requirement posttransplantation). . . . . . . . . . . . . . . . . . . . . . . . . . . . . . . . . . .

Analysis 3.6. Comparison 3 Stratified analysis, by inclusion of children, Outcome 6 Diabetes mellitus: initially diagnosed after transplantation.

Analysis 3.7. Comparison 3 Stratified analysis, by inclusion of children, Outcome 7 Post transplant lymphoproliferative disease. . . . . . . . . . . . . . . . . . . . . . . . . . . .

Analysis 3.8. Comparison 3 Stratified analysis, by inclusion of children, Outcome 8 Patients withdrawn from tacrolimus or cyclosporin. 
Analysis 4.1. Comparison 4 Stratified analysis, by studies reporting 12 month data, Outcome 1 Mortality. . . . . 60

Analysis 4.2. Comparison 4 Stratified analysis, by studies reporting 12 month data, Outcome 2 Graft loss. . . . . 61

Analysis 4.3. Comparison 4 Stratified analysis, by studies reporting 12 month data, Outcome 3 Acute rejection. $\quad$. $\quad 62$

Analysis 4.4. Comparison 4 Stratified analysis, by studies reporting 12 month data, Outcome 4 Steroid-resistent rejection. 63

Analysis 4.6. Comparison 4 Stratified analysis, by studies reporting 12 month data, Outcome 6 Diabetes mellitus: initially diagnosed after transplantation. . . . . . . . . . . . . . . . . . . . . . . . . . . . .

Analysis 4.8. Comparison 4 Stratified analysis, by studies reporting 12 month data, Outcome 8 Patients withdrawn from tacrolimus or cyclosporin. $\quad . \quad$. . . . . . . . . . . . . . . . . . . . . . . . . . . . . . . . . . . . . . . .

Analysis 5.1. Comparison 5 Stratified analysis, by studies confined to patients with hepatitis $\mathrm{C}$ virus, Outcome 1 Mortality.

Analysis 5.2. Comparison 5 Stratified analysis, by studies confined to patients with hepatitis $\mathrm{C}$ virus, Outcome 2 Graft loss. . . . . . . . . . . . . . . . . . . . . . . . . . . . . . . . . . . . . .

Analysis 5.3. Comparison 5 Stratified analysis, by studies confined to patients with hepatitis $\mathrm{C}$ virus, Outcome 3 Acute rejection.

Analysis 5.4. Comparison 5 Stratified analysis, by studies confined to patients with hepatitis C virus, Outcome 4 Steroidresistent rejection. . . . . . . . . . . . . . . . . . . . . . . . . . . . . . . . .

Analysis 5.5. Comparison 5 Stratified analysis, by studies confined to patients with hepatitis $\mathrm{C}$ virus, Outcome 5 Post transplant lymphoproliferative disease. . . . . . . . . . . . . . . . . . . . . . . . . . . . .

Analysis 5.6. Comparison 5 Stratified analysis, by studies confined to patients with hepatitis C virus, Outcome 6 Patients withdrawn from tacrolimus or cyclosporin. . . . . . . . . . . . . . . . . . . . . . . . .

Analysis 6.1. Comparison 6 Stratified analysis, by concomitant azathioprine or mycophenolate mofetil, Outcome 1 Mortality.

Analysis 6.2. Comparison 6 Stratified analysis, by concomitant azathioprine or mycophenolate mofetil, Outcome 2 Graft loss. . . . . . . . . . . . . . . . . . . . . . . . . . . . . . . . . . . . . .

Analysis 6.3. Comparison 6 Stratified analysis, by concomitant azathioprine or mycophenolate mofetil, Outcome 3 Acute rejection.

Analysis 6.4. Comparison 6 Stratified analysis, by concomitant azathioprine or mycophenolate mofetil, Outcome 4 Steroidresistent rejection. . . . . . . . . . . . . . . . . . . . . . . . . . . . . . . . . . . .

Analysis 6.5. Comparison 6 Stratified analysis, by concomitant azathioprine or mycophenolate mofetil, Outcome 5 Dialysis (de-novo requirement post-transplantation).

Analysis 6.6. Comparison 6 Stratified analysis, by concomitant azathioprine or mycophenolate mofetil, Outcome 6 Diabetes mellitus: initially diagnosed after transplantation.

Analysis 6.7. Comparison 6 Stratified analysis, by concomitant azathioprine or mycophenolate mofetil, Outcome 7 Post transplant lymphoproliferative disease.

Analysis 6.8. Comparison 6 Stratified analysis, by concomitant azathioprine or mycophenolate mofetil, Outcome 8 Patients withdrawn from tacrolimus or cyclosporin. . . . . . . . . . . . . . . . . . . . . . . . .

WHAT'S NEW $\cdot \cdot \cdot \cdot \cdot \cdot \cdot \cdot \cdot \cdot \cdot \cdot \cdot \cdot \cdot \cdot \cdot \cdot \cdot \cdot \cdot \cdot \cdot \cdot \cdot \cdot \cdot \cdot \cdot \cdot \cdot \cdot \cdot \cdot \cdot \cdot \cdot \cdot \cdot \cdot \cdot \cdot$

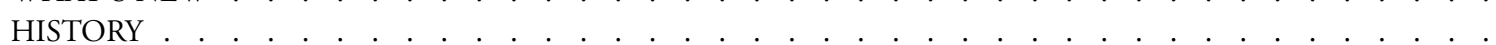

CONTRIBUTIONS OF AUTHORS . . . . . . . . . . . . . . . . . . . . . . . . . . . . . . . . . . . . . .

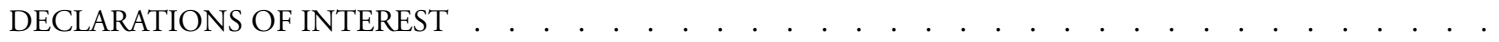

INDEX TERMS 


\title{
[Intervention Review]
}

\section{Cyclosporin versus tacrolimus for liver transplanted patients}

\author{
Elizabeth Haddad ${ }^{2}$, Vivian McAlister ${ }^{1}$, Elizabeth Renouf ${ }^{3}$, Richard Malthaner ${ }^{4}$, Mette S Kjaer ${ }^{5}$, Lise Lotte Gluud ${ }^{6}$ \\ ${ }^{1} 1$ Canadian Field Hospital, Canadian Forces, London, Canada. ${ }^{2}$ Surgery, Chatham, Canada. ${ }^{3}$ London, Canada. ${ }^{4}$ Division of Thoracic \\ Surgery, University of Western Ontario, London, Canada. ${ }^{5}$ Dept. of Hepatology A2121, Rigshospitalet, Copenhagen O, Denmark. \\ ${ }^{6}$ Cochrane Hepato-Biliary Group, Copenhagen Trial Unit, Centre for Clinical Intervention Research, Department 3344, Rigshospitalet, \\ Copenhagen University Hospital, Copenhagen, Denmark
}

Contact address: Vivian McAlister, 1 Canadian Field Hospital, Canadian Forces, C4-212, University Hospital, London, Ontario, N6A 5A5, Canada.vmcalist@uwo.ca.

Editorial group: Cochrane Hepato-Biliary Group.

Publication status and date: Edited (no change to conclusions), published in Issue 1, 2009.

Review content assessed as up-to-date: 23 August 2006.

Citation: Haddad E, McAlister V, Renouf E, Malthaner R, Kjaer MS, Gluud LL. Cyclosporin versus tacrolimus for liver transplanted patients. Cochrane Database of Systematic Reviews 2006, Issue 4. Art. No.: CD005161. DOI: 10.1002/14651858.CD005161.pub2.

Copyright (C) 2009 The Cochrane Collaboration. Published by John Wiley \& Sons, Ltd.

\section{A B S T R A C T}

\section{Background}

Most liver transplant recipients receive either cyclosporin or tacrolimus to prevent rejection. Both drugs inhibit calcineurin phosphatase which is thought to be the mechanism of their anti-rejection effect and principle toxicities. The drugs have different pharmacokinetic profiles and potencies. Several randomised clinical trials have compared cyclosporin and tacrolimus in liver transplant recipients, but it remains unclear which is superior.

\section{Objectives}

To evaluate the beneficial and harmful effects of immunosuppression with cyclosporin versus tacrolimus for liver transplanted patients.

\section{Search strategy}

The Cochrane Hepato-Biliary Group Controlled Trials Register, the Cochrane Central Register of Controlled Trials in The Cochrane Library, MEDLINE, EMBASE, and Science Citation Index Expanded, and conference proceedings were searched (August 2005) to identify relevant randomised clinical trials. Our search included scanning of reference lists in relevant articles and correspondence with investigators and pharmaceutical companies.

\section{Selection criteria}

All randomised clinical trials where tacrolimus was compared with cyclosporin for the initial treatment of first-time liver transplant recipients. We included randomised trials irrespective of blinding, language, and publication status.

\section{Data collection and analysis}

The primary outcome measure was all-cause mortality. Data were synthesised (fixed-effect model) and results expressed as relative risk (RR), values less than 1.0 favouring tacrolimus, with $95 \%$ confidence intervals (CI). Two authors assessed trials for eligibility, quality, and extracted data independently. 


\section{Main results}

We included 16 randomised trials. The number of deaths was 254 in the tacrolimus group (1899 patients) and 302 in the cyclosporin group (1914 patients). At one year, mortality (RR 0.85, 95\% CI 0.73 to 0.99 ) and graft loss (RR 0.73 , 95\% CI 0.61 to 0.86 ) were significantly reduced in tacrolimus-treated recipients. Tacrolimus reduced the number of recipients with acute rejection (RR $0.81,95 \%$ CI 0.75 to 0.88 ), and steroid-resistant rejection (RR $0.54,95 \%$ CI 0.47 to 0.74 ) in the first year. Differences were not seen with respect to lymphoproliferative disorder or de-novo dialysis rates, but more de-novo insulin-requiring diabetes mellitus (RR 1.38, 95\% CI 1.01 to 1.86) occurred in the tacrolimus group. More patients were withdrawn from cyclosporin therapy than from tacrolimus (RR 0.57 , $95 \%$ CI 0.49 to 0.66$)$.

\section{Authors' conclusions}

Tacrolimus is superior to cyclosporin in improving survival (patient and graft) and preventing acute rejection after liver transplantation, but it increases the risk of post-transplant diabetes. Treating 100 recipients with tacrolimus instead of cyclosporin would avoid acute rejection and steroid-resistant rejection in nine and seven patients, respectively, and graft loss and death in five and two patients, respectively, but four additional patients would develop diabetes after liver transplantation.

\section{PLAIN LANGUAGE SUMMARY}

Tacrolimus is superior to cyclosporin in improving patient survival, graft survival, and in preventing acute rejection after liver transplantation, but increases post-transplant diabetes

Almost every liver transplant recipient takes either cyclosporin or tacrolimus to prevent rejection of the graft. This is a review of the clinical trials that compared patients initially prescribed one of the two anti-rejection drugs after liver transplantation. Sixteen trials (3813 participants) were included. The review shows that tacrolimus is marginally better than cyclosporin at preventing patient death and graft loss. Tacrolimus is substantially better than cyclosporin at preventing rejection. No differences were seen between the drugs with respect to adverse events (renal failure, lymphoproliferative disorder) except for diabetes mellitus, which was more common with tacrolimus. After liver transplantation more patients stayed on tacrolimus than on cyclosporin. Tacrolimus is more beneficial than cyclosporine and should be considered the treatment of choice after liver transplantation. This review does not evaluate the benefit or harm of switching from one anti-rejection drug to another.

\section{B A C K G R O U N D}

Cyclosporin was introduced as primary immunosuppressant after liver transplantation instead of azathioprine over 20 years ago without testing in randomised clinical trials because of perceived transparent benefit (Starzl 1985). About 10 years ago, the first randomised clinical trials of immunosuppression after liver transplantation compared tacrolimus versus cyclosporin. Two large registration trials showed a reduction in the rate of acute rejection with tacrolimus, but reductions in post-transplantation mortality and graft loss were not statistically significant (European Study 1994; U. S. Study 1994). Subsequently both tacrolimus and cyclosporin were found to have a common mechanism of action (ie, inhibition of calcineurin phosphatase) even though they bound different intra-cellular proteins. These intra-cellular proteins be- long to the immunophilin family. Cyclosporin binds cyclophilin and tacrolimus binds FKBP12 (Siekierka 1992). The role of immunophilin binding in the mechanism of toxicity is not clear, but it may allow for a different side effect profile of these drugs, which are now known as calcineurin inhibitors. For instance, cyclosporin is known to cause hirsuitism whereas tacrolimus either has no effect or causes hair loss. Therefore, cyclosporin and tacrolimus may have different benefit and harm profiles, but to date randomised clinical trials have not shown statistically significant differences in major outcomes after liver transplantation. A systematic review of cyclosporin versus tacrolimus for kidney transplanted patients has been conducted (Knoll 1999; Webster 2005), but we have not identified previous meta-analyses or systematic reviews for liver 
transplanted patients (Knoll 1999).

\section{O B J E C T I VES}

To evaluate the beneficial and harmful effects of immunosuppression with cyclosporin versus tacrolimus for liver transplanted patients.

\section{METHODS}

\section{Criteria for considering studies for this review}

\section{Types of studies}

Randomised clinical trials were included irrespective of language or publication status (ie, unpublished trials, abstracts, or full paper articles).

\section{Types of participants}

We included patients undergoing their first liver transplantation. Patients were excluded if they were undergoing multi-organ transplantation, had previously received a liver transplant, or were receiving an ABO-incompatible transplant.

\section{Types of interventions}

We included randomised comparisons of tacrolimus versus cyclosporin. The dose and duration of therapy were considered in our inclusion criteria, but were to be evaluated in subgroup analyses. Collateral interventions were allowed if received by all intervention arms with the exception of azathioprine administration, which was to be evaluated in subgroup analyses.

\section{Types of outcome measures}

The following outcome measures were evaluated one year after randomisation.

(1) All-cause mortality (primary outcome measure)

(2) Graft loss

(3) Rejection

(4) Steroid-resistant rejection (as defined by each study)

(5) New-onset diabetes (as defined by each study)

(6) New-onset dialysis-dependent renal failure

(7) Post-transplant lymphoproliferative disease

(8) Dose reductions due to adverse events

(9) Withdrawals and dropouts.

In studies where one year follow-up was not available even after correspondence with the principal investigator, those outcomes that are available at the nearest time point to one year were included in the general and sub-group analyses.

\section{Search methods for identification of studies}

S Klingenberg, the Trials Search Coordinator, performed electronic searches using search strategies as revised by the authors. E Haddad and V McAlister evaluated whether these trials fulfilled the inclusion criteria. The search results and selections were monitored by all authors.

The Cochrane Hepato-Biliary Group Controlled Trials Register (August 2005), the Cochrane Central Register of Controlled Trials (CENTRAL) in The Cochrane Library (Issue 3, 2005), MEDLINE (1950 to August 2005), EMBASE (1980 to August 2005), and Science Citation Index Exapanded (1945 to August 2005) (Royle 2003) were searched using the strategies as described in Appendix 1. E Renouf scanned bibliographies in relevant articles and conference proceedings (Transplantation Society biannual meetings 1988 to 2004; International Liver Transplantation Society Congress 1995 to 2004). V McAlister wrote to authors of included trials and pharmaceutical companies that are involved in the production of tacrolimus or cyclosporin.

\section{Data collection and analysis}

The present review was performed following the recommendations of The Cochrane Collaboration (Higgins 2005). Identified trials were listed and their fulfilment of the inclusion criteria assessed by V McAlister. Excluded trials were listed with the reason for exclusion.

\section{Data extraction}

E Haddad and V McAlister independently extracted data using standardised extraction forms. E Renouf prepared copies of the reports that were blinded with regard to place of publication, authors, and their affiliation for the primary data extraction process. Data extraction results were monitored by all authors. Disagreements were resolved by discussion. V McAlister wrote to investigators and to the sponsoring companies of included trials to ask for data that were not presented in the published reports.

From each trial, we extracted the following characteristics of the included:

- Patients (inclusions and exclusion criteria, mean age, proportion of men, aetiology of liver disease, creatinine pre-op and at one year;

- Interventions (dose and duration, concomitant therapy, maintenance drug dose, maintenance drug level);

- Trials (setting, methodological quality, publication status, duration of follow-up, and all outcomes).

Methodological quality 
Randomisation and follow-up were extracted as measures of methodological quality (Kjaergard 2001) using the definitions listed below.

\section{Generation of the allocation sequence}

This is the procedure used to create a random sequence ensuring that each patient has a known, unpredictable, and usually equal chance of being assigned to intervention groups. The allocation sequence generation was classified as adequate (if the allocation sequence was generated by a computer or random number table), unclear (if the trial was described as randomised, but the method used for the allocation sequence generation was not described), or inadequate (if a system involving dates, names, or admittance numbers were used).

\section{Allocation concealment}

This is the procedure used to conceal the allocation sequence from the investigators who assign patients to the intervention groups. The allocation concealment was classified as adequate (if the allocation of patients involved a central independent unit, sealed envelopes, on-site locked computer, or identically appearing numbered drug bottles or containers prepared by an independent pharmacist), unclear (if the trial was described as randomised, but the method used to conceal the allocation was not described), or inadequate (if the trial was quasi-randomised).

\section{Blinding}

Considering the nature of the intervention, we expected that none of the eligible trials will be double blind. Blinding was therefore not included in our assessment of methodological quality.

\section{Follow -up}

We extracted the number and reasons for all losses to follow-up to assess the risk of attrition bias.

\section{Statistical analyses}

The analyses were performed in RevMan Analysis 1.0 (RevMan 2003) and Stata version 6.0 for Windows. The number of events and number of patients in all intervention arms were used to calculate relative risks (RR) with 95\% confidence intervals (CI). Risk differences with 95\% CI were also analysed and tabulated for outcome differences. Continuous outcomes were presented as weighted mean differences (WMD) with 95\% CI. Because we expected considerable homogeneity, data were combined in fixed-effect meta-analyses. Random-effects meta-analyses were performed as sensitivity analyses, but were only to be reported if the results differ significantly from the fixed-effect models. Intention-to-treat analyses including all patients irrespective of compliance or followup was performed. Carry forward of the last observed response was used for patients with missing data. For the primary outcome measure, evidence of publication bias and other biases was evaluated in regression analyses of funnel plot asymmetry. Sources of heterogeneity were evaluated through sensitivity, subgroup, and metaregression analyses. The analyses included the extracted patient, intervention, and trial characteristics listed above as explanatory variables. V McAlister performed all meta-analyses in RevMan. LL
Gluud performed additional blinded statistical analyses including meta-regression and regression analyses of funnel plot asymmetry. The following subgroup analyses were carried out: paediatric recipients; patients infected with hepatitis $C$ virus at the time of transplantation; trials using oil-based cyclosporine; trials where cyclosporine was combined with azathioprine; trials where tacrolimus and cyclosporine are combined with mycophenolate mofetil or sirolimus. Sensitivity analyses that combine outcomes from trials where follow-up data for one year were not available, even after correspondence with the principle investigator, were performed using a time point closest to one year (either less or more) that was available.

\section{R E S U L T S}

\section{Description of studies}

See: Characteristics of included studies; Characteristics of excluded studies.

Searches performed on August 30, 2005 resulted in 717 hits. This yielded 114 reports when duplicates were removed. After initial review, 20 randomised trials were identified of which four were excluded on further examination because they were a review of other studies (Arnold 1995), a sub-analysis of another study (Loinaz 2001), or designed for other purposes, usually regarding perioperative care, without any of the outcomes being studied for inclusion in this systematic review (Ericzon 1997; Trull 2002). Data at one year after liver transplantation were available in all the remaining 16 trials, except for two in which data were only available at three months (Timmermann 2002) and at six months(Stegall 1997). In one trial data were available at one year with the exception of graft loss, which was only available at six months (Grazi 2004). After contacting principal investigators and sponsoring pharmaceutical companies supplementary information was supplied regarding eight reports (Fung 1991; Fisher 1998; Klupp 1999; Loinaz 2001; Muehlbacher 2001; Therapondos 2002; Greig 2003; Grazi 2004).

\section{Included trials}

The 16 included trials allocated 3813 participants of whom 1899 were randomised to tacrolimus and 1914 to cyclosporin. Seven of the trials were conducted at single centre sites (Fung 1991; Stegall 1997; Fisher 1998; Zervos 1998; Klupp 1999; Rolles 1999; Therapondos 2002), while the remaining nine trials were multicentred. In all, the trials involved 59 liver transplantation centres in 18 countries. Most of the randomised trials restricted enrolment to adults, but one included children (U. S. Study 1994) and one was restricted to children (Kelly 2004). Hepatitis C virus (HCV) cirrhosis was the commonest indication for transplantation in studies after 1994; two randomised trials confined entry to patients with 
hepatitis C virus (HCV) (Zervos 1998; Martin 2004); only one other trial identified the outcome in patients with HCV (Grazi 2004). The three earliest trials with 1157 participants compared tacrolimus with the original oil based formulation of cyclosporin (Sandimmun ${ }^{\circledR}$ ) (Fung 1991; European Study 1994; U. S. Study 1994) whereas the other 15 trials with 1656 participants compared tacrolimus with the microemulsion formulation (Neoral $\left.{ }^{\circledR}\right)$. Concomitant immunosuppression given to all patients (both study groups, all participating centres) included corticosteroids (all trials except Rolles 1999); azathioprine (O'Grady 2002; Therapondos 2002; Greig 2003; Martin 2004), and mycophenolate mofetil ( Stegall 1997; Fisher 1998; Klupp 1999). In one multi-centred trial, azathioprine was given only in some centres but to both study groups (Grazi 2004). In five trials, azathioprine was given only to cyclosporin treated patients according to local practice (European Study 1994; U. S. Study 1994; Muehlbacher 2001; Timmermann 2002; Kelly 2004). All of the trials used trough level monitoring to guide cyclosporin and tacrolimus dosing except one trial (Grazi 2004), which used the two hour post-dose level to guide the dose of cyclosporin.

\section{Risk of bias in included studies}

Allocation concealment was adequate in most trials. The method of allocation concealment was not specified in three trials (Stegall 1997; Zervos 1998; Rolles 1999). All of the trials were open labelled because of the need for therapeutic drug monitoring. Intention-to-treat analysis was available in all the trials. Follow-up data were complete for patient and graft survival (primary outcome) and for rejection, but reduced in all other categories. In one trial graft survival was only reported at six months, but all other data were available at 12 months after transplantation (Grazi 2004).

\section{Effects of interventions}

The primary outcome favoured tacrolimus. Mortality at one year was reduced by $15 \%$ in the tacrolimus patients (comparison 01.01 : RR $0.85,95 \%$ CI 0.73 to 0.99 ). Graft survival was reported in 15 trials favouring tacrolimus with $22 \%$ less grafts lost (comparison 01.02: RR 0.78, 95\% CI 0.68 to 0.89 ). Rejection and steroid resistant rejection were reduced by $18 \%$ and $43 \%$, respectively, in the tacrolimus treated recipients (comparison 01.03: RR 0.82, 95\% CI 0.77 to 0.88 ; comparison 01.04 : RR $0.57,95 \%$ CI 0.46 to 0.71$)$. These results are from intention-to-treat analyses. Substantially more patients discontinued cyclosporin than tacrolimus (comparison 01.10: RR 0.65, 95\% CI 0.57 to 0.74 ). However, the rate of new-onset diabetes was increased by $27 \%$ in the tacrolimustreated patients (comparison 01.08: RR 1.27, 95\% CI 1.12 to 1.44).

No differences were seen in the rates of chronic renal failure requiring dialysis ((comparison 01.05: RR 1.55, 95\% CI 0.64 to 3.78) or of lymphoproliferative disorder after liver transplantation (comparison 01.09: RR 1.01, 95\% CI 0.36 to 2.86). Differences in the serum creatinine at one year favouring tacrolimus were not statistically significant, but data were available from only two trials with a total of 672 patients (comparison 01.07: RR, -2.67 $\mathrm{mmol} / \mathrm{L}, 95 \%$ CI -9.55 to 4.22 ). Insufficient data were reported regarding other adverse events for systematic analysis, but the data are included in the characteristics of included trials table.

The number of deaths was 254 in the tacrolimus group (1899 patients) and 302 in the cyclosporin group (1914 patients). The actual number of patients and events are presented in Table 1 with the absolute risk differences and 95\% CI. Treating 100 recipients with tacrolimus instead of cyclosporin would avoid rejection and steroid-resistant rejection in nine and seven patients, respectively, and graft loss and death in five and two patients, respectively, but four additional patients would develop diabetes after liver transplantation.

Table 1. Number of patients (N), events (n), risk difference, $95 \%$ confidence interval CI

\begin{tabular}{|c|c|c|c|c|c|}
\hline Event & $\begin{array}{l}\text { Tacrolimus } \quad n / N \\
(\%)\end{array}$ & $\begin{array}{l}\text { Cyclosporin } \quad n / N \\
(\%)\end{array}$ & risk difference & 95\% CI minimum & 95\% CI maximum \\
\hline Death & 254 / 1899 (13.4\%) & $302 / 1914(15.8 \%)$ & $-2 \%$ & $0 \%$ & $-5 \%$ \\
\hline Graft loss & $281 / 1654(17.0 \%)$ & 365 / $1664(21.9 \%)$ & $-5 \%$ & $-2 \%$ & $-8 \%$ \\
\hline Acute rejection & $720 / 1865(38.6 \%)$ & $885 / 1881(47.0 \%)$ & $-9 \%$ & $-6 \%$ & $-12 \%$ \\
\hline $\begin{array}{l}\text { Steroid-resistant re- } \\
\text { jection }\end{array}$ & $110 / 1193(9.2 \%)$ & $205 / 1246(16.5 \%)$ & $-7 \%$ & $-4 \%$ & $-9 \%$ \\
\hline $\begin{array}{l}\text { Drug discontinua- } \\
\text { tion }\end{array}$ & $222 / 1573(14.1 \%)$ & $392 / 1583(24.8 \%)$ & $-11 \%$ & $-8 \%$ & $-13 \%$ \\
\hline
\end{tabular}


Table 1. Number of patients (N), events (n), risk difference, $\mathbf{9 5 \%}$ confidence interval CI (Continued)

\begin{abstract}
Diabetes $\quad 306 / 1503(20.4 \%) \quad 242 / 1520(15.9 \%)$
Regression asymmetry tests showed no significant evidence of publication bias or other biases $(\mathrm{P}=0.33)$. In meta-regression analyses the treatment effect was not significantly associated with the allocation sequence generation (regression coefficient -0.022 , $95 \%$ CI -0.57 to 0.52 ) or allocation concealment (regression coefficient $-0.17,95 \% \mathrm{CI}-0.48$ to 0.15$)$. Identical results for the primary outcome were found whether fixed-effect or random-effects meta-analyses were used because the studies lack heterogeneity, I $2=0 \%$. Random-effects meta-analyses did not change the other outcomes.
\end{abstract}

Stratified analyses showed that the heterogeneity of the trials and the outcomes were not altered by inclusion of the following subgroups: (1) oil-based cyclosporin (comparison 02) (Fung 1991; European Study 1994; U. S. Study 1994); (2) trials with children (comparison 03) (U. S. Study 1994; Kelly 2004), and (3) trials not reporting 12 month data (comparison 04) (Stegall 1997; Timmermann 2002; Grazi 2004). Separate analyses of these subgroups showed similar results to the rest of the trials except that some subgroups were of insufficient size for the differences to be statistically significant. Stratified analysis of trials confined to patients with HCV (Zervos 1998; Martin 2004) did not alter the result when combined with the other trials, all of which included patients with HCV and other diagnoses (comparison 05). Stratified analysis of the different protocols of concomitant immunosuppression with azathioprine or mycophenolate mofetil also showed similar results in each subgroup, but the sample sizes were much reduced by the stratification (comparison 06). Reporting of actual doses and levels of drug used was too sparse to permit more detailed analysis, and the results are given in the characteristics table of each trial. Similarly, other adverse events, which were not reported regularly enough for comparison, are reported in the characteristics table of each trial.

\section{DISCUSSION}

Cyclosporin was introduced, without the benefit of clinical trial, into care of the recipient after liver transplantation. Even at the time that tacrolimus was developed, considerable hesitancy remained regarding the robustness of liver transplantation to allow for randomisation. This concern is apparent in a description of the earliest randomised trial in this review (Fung 1991) and in a discus- sion of the two registration randomised clinical trials of tacrolimus (Starzl 1995). In the succeeding decade, a further 13 randomised clinical trials of tacrolimus versus cyclosporin in liver transplantation were performed indicating increasing comfort with the procedure. This systematic review shows why this occurred. Outcomes after liver transplantation are very good. Overall patient and graft survival rates are $85 \%$ and $80 \%$, respectively.

The superiority of tacrolimus over cyclosporin after liver transplantation has to be considered in the context of these excellent overall results. Calculating the risk difference of each treatment helps us understand its impact. Treating 100 liver recipients with tacrolimus instead of cyclosporin would result in two less deaths, five less graft losses, nine less patients with acute rejection, and seven less with steroid-resistant rejection, but four more patients would develop diabetes. The ranges suggested by $95 \%$ CI for these risk differences are included in Table 1.

More heterogeneity between the trials is seen in rejection than in other outcomes. One source of heterogeneity here may be the variable rates of rejection observed in each trial. This is probably due to the evolution of rejection diagnosis over time. Definition of rejection was standardised within trials, but not between trials. The rate of diagnosis of rejection appears to be lower in more recent trials than in earlier ones, even though the immunosuppressive protocols are similar. This might be due to improved differential diagnostic ability and a reduced liver biopsy rate, but these features are beyond the scope of this review. There was no evidence that different criteria were applied to recipients of either tacrolimus or cyclosporin in the trials. Despite the heterogeneity, a consistent finding in each trial was a clinically and statistically significantly lower rate of rejection in liver transplant recipients randomised to tacrolimus.

Relatively few trials excluded adjuvant immunosuppression with either azathioprine or mycophenolate mofetil. Several trials permitted use of azathioprine in cyclosporin, but not tacrolimus treated patients at centres where this was the normal practice. One trial permitted this use of azathioprine at certain centres only if it were also prescribed to tacrolimus recipients. More recently trials of concomitant mycophenolate mofetil given to both groups have been performed. Stratification of the analyses according to the different forms of adjuvant immunosuppression reduced the sample size so that conclusions cannot be drawn with respect to mortality, 
but most of the other comparisons did not show any impact of adjuvant immunosuppression on the differences reported above between cyclosporin and tacrolimus.

The outcomes studied are reported on the basis of intention to treat. Cyclosporin discontinuation does not ameliorate the disadvantage associated with that group. The benefit of switching from cyclosporin to tacrolimus or vice versa cannot be evaluated from these trials. This review looked at outcomes one year after transplantation. The impact of diabetes may not be manifest for many years. The risk of post-transplant lymphoproliferative disorder persists beyond one year, so that outcomes for the groups with respect to that comparison may change. Similarly the risk of calcineurin inhibitor related nephrotoxicity may increase with time. The results of this review must be considered in the context of possible long-term outcomes.

The involvement of the majority of transplant centres throughout the world in the randomised trials reviewed combined with the lack of heterogeneity of the trials support the veracity of this review. Very similar results with respect to graft survival, rejection, and diabetes were achieved by a recent Cochrane review of tacrolimus and cyclosporin in kidney transplantation (Webster 2005). Both cyclosporin and tacrolimus are immunosuppressive because they inhibit calcineurin phosphatase in lymphocytes. Inhibition of the same pathway in the beta-cells of the pancreas reduces insulin production. The superior effect of tacrolimus in the prevention of rejection was accompanied by an increase in the rate of diabetes in this review and in the kidney transplantation metaanalysis (Webster 2005). The difference between cyclosporin and tacrolimus may, therefore, be related to the potency of calcineurin phosphatase inhibition. Insufficient data regarding exposure to cyclosporin or tacrolimus were reported to know if the outcomes would merge with particular dosing protocols. The higher rate of cyclosporin discontinuation seen consistently throughout the re- views suggests that clinicians find it more difficult to achieve the balance between efficacy and unwanted effects with that medication than with tacrolimus. Differences between cyclosporin or tacrolimus in pharmacokinetic profiles or in secondary adverse events may account for this difficulty.

\section{A U T HOR S' CONCLUSIONS Implications for practice}

Tacrolimus is superior to cyclosporin in patients after liver transplantation. Liver transplant recipients on tacrolimus need careful monitoring for the development of diabetes.

\section{Implications for research}

More research with cyclosporin and tacrolimus is required to disassociate the unwanted effects of calcineurin inhibition from the intended immunosuppressive effect. These investigations may exploit the different pharmacokinetic profiles, different drug interactions, and different adverse effect profiles of cyclosporin and tacrolimus to maintain maximum patient and graft survival with minimum adverse events.

\section{ACKNOWLEDGEMENTS}

Our thanks to Sarah Klingenberg, the Trials Search Coordinator of The Cochrane Hepato-Biliary Group for suggesting search strategies and performing electronic searches in the Controlled Trials Register of The Cochrane Library, to Dimitrinka Nikolova for excellent co-ordination, and to Christian Gluud for advice and support. We are grateful to the editor and reviewers for their suggestions at each stage of the review.

\section{RE FER E NCE S}

\section{References to studies included in this review}

\section{European Study 1994 \{published data only\}}

European FK506 Multicentre Liver Study Group. Randomised trial comparing tacrolimus (FK506) and cyclosporin in prevention of liver allograft rejection. Lancet 1994;344:423-8. [MEDLINE: 7520105]

Fisher 1998 \{published and unpublished data\}

* Fisher RA, Ham JM, Marcos A, Shiffman ML, Luketic VA, Kimball PM, et al.A prospective randomized trial of mycophenolate mofetil with neoral or tacrolimus after orthotopic transplantation. Transplantation 1998;66(12):1616-21. [MEDLINE: 9884248] Fisher RA, Stone JJ, Wolfe LG, Rodgers CM, Anderson ML, Sterling RK, et al.Four-year follow-up of a prospective randomized trial of mycophenolate mofetil with cyclosporine microemulsion or tacrolimus following liver transplantation. Clinical Transplantation 2004;18(4):436-72. [MEDLINE: 15233827]

Fung 1991 \{published and unpublished data\}

* Fung J, Abu-Elmagd K, Jain A, Gordon R, Tzakis A, Todo S, et al.A randomized trial of primary liver transplantation under immunosuppression with FK 506 vs cyclosporine. Transplantation Proceedings 1991;23(6):2977-83. [MEDLINE: 172133]

Fung JJ, Eliasziw M, Todo S, Jain A, Demetris AJ, McMichael JP, et al.The Pittsburgh randomized trial of tacrolimus compared to cyclosporine for hepatic transplantation. Journal of the American College of Surgeons 1996;183(2):117-25. [MEDLINE: 8696542]

Grazi 2004 \{published and unpublished data\}

* Grazi GL, Levy G, Wu Y, Marotta P, Boillot O, Sanjuan F, et al.12-month follow-up data from a randomized multicentre, prospective study of cyclosporine C2 monitoring versus tacrolimus 
in liver transplantation (LIS2T). American Journal of

Transplantation 2004;4(Suppl 8):268.

Levy G, Villamil F, Samuel D, Sanjuan F, Grazi GL, Wu Y, et al.Results of LIS2T, a multicenter, randomized study comparing cyclosporine microemulsion with $\mathrm{C} 2$ monitoring and tacrolimus with $\mathrm{C} 0$ monitoring in de novo liver transplantation.

Transplantation 2004;77(11):1632-8. [MEDLINE: 15201658]

Greig 2003 \{published and unpublished data\}

Greig P, Lilly L, Scudamore C, Erb S, Yoshida E, Kneteman N, et al.Early steroid withdrawal after liver transplantation: the Canadian tacrolimus versus microemulsion cyclosporin A trial. 1-year followup. Liver Transplantation 2003;9(6):587-95. [MEDLINE: 12783400]

Kelly 2004 \{published data only\}

Kelly D, Jara P, Rodeck B, Lykavieris P, Burdelski M, Becker M, et al.Tacrolimus and steroids versus ciclosporin microemulsion, steroids, and azathioprine in children undergoing liver transplantation: randomised European multicentre trial. Lancet 2004;364:1054-61. [MEDLINE: 15380964]

Klupp 1999 \{published and unpublished data\}

Klupp J, Glanemann M, Bechstein WO, Platz KP, Langrehr JM, Keck H, et al.Mycophenolate mofetil in combination with tacrolimus versus neoral after liver transplantation. Transplantation Proceedings 1999;31:1113-4. [MEDLINE: 10083497]

Martin 2004 \{published data only\}

Martin P, Busuttil RW, Goldstein RM, Crippin JS, Klintmalm GB, Fitzsimmons WE, et al.Impact of tacrolimus versus cyclosporine in hepatitis $\mathrm{C}$ virus-infected liver transplant recipients on recurrent hepatitis: a prospective randomized trial. Liver Transplantation 2004;10(10):1258-62. [MEDLINE: 15376310]

Muehlbacher 2001 \{published and unpublished data\}

* Muehlbacher FF, for the European Liver Transplantation Tacrolimus vs Cyclosporin Microemulsion Study Group.

Tacrolimus versus cyclosporin microemulsion in liver transplantation: results of one-year follow-up. 10th ESOT \& 12th ETCO Congress 2001, October 6-11, 2001, Lisboa, Portugal. 2001.

Muhlbacher F, European Liver Transplantation Tacrolimus vs Cyclosporin Microemulsion Study Group. Tacrolimus versus cyclosporin microemulsion in liver transplantation: results of a 3month study. Transplantation Proceedings 2001;33(1-2):1339-40. [MEDLINE: 11267317]

O'Grady 2002 \{published data only\}

O'Grady JG, Burroughs A, Hardy P, Elbourne D, Truesdale A, The UK and Republic of Ireland Liver Transplant Study Group.

Tacrolimus versus microemulsified ciclosporin in liver transplantation: the TMC randomised controlled trial. Lancet 2002;360:1119-25. [MEDLINE: 12387959]

Rolles 1999 \{published data only\}

Rolles K, Davidson BR, Burroughs AK. A pilot study of immunosuppressive monotherapy in liver transplantation: tacrolimus versus microemulsified cyclosporin. Transplantation 1999;68(8):1195-209. [MEDLINE: 10551650]

Stegall 1997 \{published data only\}

Stegall M, Wachs ME, Everson G, Steinberg T, Bilir B, Shrestha R, et al.Prednisone withdrawal 14 days after liver transplantation with mycophenolate: a prospective trial of cyclosporine and tacrolimus. Transplantation 1997;64(12):1755-60. [MEDLINE: 9422416]

Therapondos 2002 \{published and unpublished data\}

Therapondos G, Flapan AD, Dollinger MM, Garden OJ, Plevris JN, Hayes PC. Cardiac function after orthotopic liver transplantation and the effects of immunosuppression: a prospective randomized trial comparing cyclosporin (Neoral) and tacrolimus. Liver Transplantation 2002;8(8):690-700.

[MEDLINE: 12149762]

\section{Timmermann 2002 \{published data only\}}

Timmermann W, Erhard J, Lange R, Reck T, Kockerling F, Muller A, et al.A randomised trial comparing the efficacy and safety of tacrolimus with microemulsified cyclosporine after liver transplantation. Transplantation Proceedings 2002;34(5):1516-8. [MEDLINE: 12176463]

U. S. Study 1994 \{published data only\}

The US Multicentre FK506 Study Group. A comparison of tacrolimus (FK 506) and cyclosporine for immunosuppression in liver transplantation. New England Journal of Medicine 1994;331 (17):1110-5. [MEDLINE: 7523946]

Zervos 1998 \{published and unpublished data\} Zervos XA, Weppler D, Fragulidis GP, Torres MB, Nery JR, Khan $\mathrm{MF}$, et al.Comparison of tacrolimus with microemulsion cyclosporine as primary immunosuppression in hepatitis $\mathrm{C}$ patients after liver transplantation. Transplantation 1998;65(8):1044-6. [MEDLINE: 9583863]

\section{References to studies excluded from this review}

Arnold 1995 \{published data only\}

Arnold JC, Theilmann L. Comparison between tacrolimus (FK 506) and cyclosporin in immunosuppressive therapy after liver transplantation [Vergleich zwischen Tacrolimus (FK 506) und Cyclosporin in der immunosuppressiven Therapie nach Lebertransplantation]. Zeitschrift fur Gastroenterologie 1995;33(10): 624-5. [MEDLINE: 7502559]

Ericzon 1997 \{published data only\}

Ericzon B, Eusufzai S, Soderdahl G, Duraj F, Einarsson K, Angelin B. Secretion and composition of bile after human liver transplantation. Transplantation 1997;63(1):74-80. [MEDLINE: 9000664]

Loinaz 2001 \{published and unpublished data\}

Loinaz C, Marin LM, Gonzalez-Pinto I, Gomez R, Jimenez C, Moreno E. A single-centre experience with cyclosporine microemulsion versus tacrolimus in 100 randomized liver transplant recipients: midterm efficacy and safety. Transplantation Proceedings 2001;33:3439-41. [MEDLINE: 11750472]

Trull 2002 \{published data only\}

Trull A, Hughes V, Cooper D, Wilkins M, Gimson A, Friend P, et al.Influence of albumin supplementation on tacrolimus and cyclosporine therapy early after liver transplantation. Liver Transplantation 2002;8(3):224-32. [MEDLINE: 11910567]

\section{Additional references}




\section{Higgins 2005}

Higgins JPT, Green S, editors. Cochrane Handbook for Systematic Reviews of Interventions 4.2.5 [updated May 2005]. The Cochrane Library, Issue 3, 2005. Chichester, UK: John Wiley \& Sons, Ltd.

\section{Kjaergard 2001}

Kjaergard LL, Villumsen J, Gluud C. Reported methodologic quality and discrepancies between large and small randomized trials in meta-analyses. Annals of Internal Medicine 2001;135(11):982-9. [MEDLINE: 11730399]

\section{Knoll 1999}

Knoll GA, Bell RC. Tacrolimus versus cyclosporin for immunosuppression in renal transplantation: meta-analysis of randomised trials. BMJ (Clinical Research Ed.) 1999;318(7191): 1104-7. [MEDLINE: 10213717]

\section{RevMan 2003}

Copenhagen: The Nordic Cochrane Centre, The Cochrane Collaboration. Review Manager (RevMan). 4.2 for Windows. Copenhagen: The Nordic Cochrane Centre, The Cochrane Collaboration, 2003.

\section{Royle 2003}

Royle P, Milne R. Literature searching for randomized controlled trials used in Cochrane reviews: rapid versus exhaustive searches. International Journal of Technology Assessment in Health Care 2003; 19(4):591-603.

\section{Siekierka 1992}

Siekierka JJ, Sigal NH. FK-506 and cyclosporin A: immunosuppressive mechanism of action and beyond. Current Opinion in Immunology 1992;4(5):548-52. [MEDLINE: 1384551]

\section{Starzl 1985}

Starzl TE, Iwatsuki S, Shaw BW Jr, Gordon RD, Esquivel CO. Immunosuppression and other nonsurgical factors in the improved results of liver transplantation. Seminars in Liver Disease 1985;5(4): 334-43. [MEDLINE: 3909427]

\section{Starzl 1995}

Starzl TE, Donner A, Eliasziw M, Stitt L, Meier P, Fung JJ, et al.Randomised trialomania? The multicentre liver transplant trials of tacrolimus. Lancet 1995;346(8986):1346-50. [MEDLINE: 7475777]

\section{Webster 2005}

Webster A, Woodroffe R, Taylor R, Chapman J, Craig J.

Tacrolimus versus cyclosporin as primary immunosuppression for kidney transplant recipients. The Cochrane Database of Systematic Reviews 2005, Issue 4. Art. No.: CD003961. DOI: 10.1002/ 14651858.CD003961.pub2.

* Indicates the major publication for the study 
CHARACTERISTICS OF STUDIES

\section{Characteristics of included studies [ordered by study ID]}

\section{European Study 1994}

Gethods
of 4 , each block containing an equal number allocated to the 2 treatment groups; stratified for the presence
of fulminant hepatic failure.
Allocation concealment: adequate. Third party allocation.
Blinding: not performed.
Follow-up: adequate.

Participants

Country: Eight centres in four European countries.

Language: English.

Inclusion criteria: Male and female patients, aged 18 to 70 , undergoing primary isolated liver transplantation.

Exclusion criteria / diagnoses: vasculitis, primary liver cancer with metastases, HIV, treatment with an investigational agent.

Allocation: tacrolimus $\mathrm{n}=264$; cyclosporin $\mathrm{n}=265$.

Interventions
Tacrolimus protocol:
- $0.075 \mathrm{mg} / \mathrm{kg}$ IV over 4 hours $\mathrm{q} 12 \mathrm{~h}$ for 3 days then conversion to oral at $0.30 \mathrm{mg} / \mathrm{kg} / \mathrm{d}$
- during the study changes to the tacrolimus regimen resulted in a lower daily dose
- all tacrolimus patients also received IV methylprednisolone at $10 \mathrm{mg} / \mathrm{kg}$ intra-op or post-op (single dose)
followed by $20 \mathrm{mg} / \mathrm{d}$ prednisolone or equivalent methylprednisolone if patient unable to take oral
- steroids tapered and withdrawal was acceptable.
Cyclosporin protocol:
- cyclosporin formulation: oil-based
- centre dependent - 1 to $6 \mathrm{mg} / \mathrm{kg}$ IV or 8 to $15 \mathrm{mg} / \mathrm{kg}$ oral
- all cyclosporin patients also received azathioprine from $1 \mathrm{to} 3 \mathrm{mg} / \mathrm{kg}$, steroids from 0.5 to $2.0 \mathrm{mg} / \mathrm{kg}$
- in three cantres the cyclosporin patients received ATG $5 \mathrm{mg} / \mathrm{kg} / \mathrm{d} \mathrm{for} 1$ week .
Concomitant immunosuppression: steroids to all patients; antithymocyte globulin to both groups at 3
centres; azathioprine to cyclosporin recipients according to local practice.

\begin{tabular}{ll}
\hline Outcomes & - patient survival \\
& - graft survival \\
& - acute rejection \\
& - refractory rejection \\
& - chronic rejection \\
& - insulin dependent DM \\
& - creatinine \\
& - infection \\
& - withdrawal \\
& - impaired renal function \\
& - neurologic complications \\
& - hirsutism \\
\hline Notes & Follow-up: 12 months \\
& Other adverse events: \\
& tremor - tacrolimus (127/264); cyclosporin $(85 / 265)$ \\
\hline
\end{tabular}




\section{European Study 1994 (Continued)}

paraesthesia - tacrolimus (45/264), cyclosporin (44/265)

infection - tacrolimus (99/264); cyclosporin (107/265)

cytomegalovirus - tacrolimus (41/264); cyclosporin (58/265)

pneumonia - tacrolimus (43/264); cyclosporin (56/265).

\section{Risk of bias}

\begin{tabular}{l|ll}
\hline Item & Authors' judgement & Description \\
\hline Allocation concealment? & Yes & A - Adequate \\
\hline
\end{tabular}

Fisher 1998

Methods

Generation of the allocation sequence: adequate. Random numbers.

Allocation concealment: adequate. Third party allocation.

Blinding: not performed.

Follow-up: adequate.

Analysis: intention to treat.

\begin{tabular}{ll}
\hline Participants & Country: USA. \\
& Language: English. \\
& Inclusion criteria: adult patients, male and female, undergoing orthotopic liver transplantation. \\
& Exclusion criteria: not specified. \\
& Allocation: tacrolimus $\mathrm{n}=49 ;$ cyclosporin $\mathrm{n}=50$. \\
\hline
\end{tabular}

\begin{tabular}{|c|c|}
\hline Interventions & $\begin{array}{l}\text { Tacrolimus protocol: } \\
\text { - } 0.15 \mathrm{mg} / \mathrm{kg} / \mathrm{d} \text { orally twice per day started on day } 2 \text { to maintain blood levels of } 10 \text { to } 15 \mathrm{ng} / \mathrm{ml} \text { for } 2 \\
\text { months and } 5 \text { to } 10 \mathrm{ng} / \mathrm{ml} \text { thereafter. } \\
\text { Cyclosporin protocol: } \\
\text { - cyclosporin formulation: microemulsion } \\
\text { - cyclosporin } 8 \text { to } 10 \mathrm{mg} / \mathrm{kg} / \mathrm{d} \text { orally twice per day starting on day } 2 \text { to maintain blood level of } 300 \text { to } \\
400 \mathrm{ng} / \mathrm{ml} \text { for } 2 \text { months and } 200 \text { to } 300 \mathrm{ng} / \mathrm{ml} \text { thereafter: } \\
\text { Concomitant immunosuppression: steroids all patients; MMF all patients. } \\
\text { - all patients received } 1.5 \mathrm{~g} \text { of MMF orally } 4-6 \mathrm{hrs} \text { prior to transplantation; during } 1 \mathrm{st} \text { week MMF at } 3 \\
\text { g/d if blood loss }<10 \mathrm{U} \text { and } 2 \mathrm{~g} / \mathrm{d} \text { if }>=10 \mathrm{U} \text {; reduced to } 2 \mathrm{~g} / \mathrm{d} \text { after } 7 \text { days and } 1 \mathrm{~g} / \mathrm{d} \text { by } 6 \mathrm{months} \text { ( } \\
\text { decreased by } 50 \% \text { or discontinued of WBC }<4 \text { ) } \\
\text { - prednisone was tapered to } 20 \mathrm{mg} / \mathrm{d} \text { by day } 18,7.5 \mathrm{mg} / \mathrm{d} \text { by } 3 \text { months, } 5 \mathrm{mg} / \mathrm{d} \text { by } 6 \text { months } \\
\text { - patients received PGE } 1 \text { as a continuous central venous infusion over } 5 \text { to } 7 \text { days beginning at } 10 \mathrm{ug} / \mathrm{hr} \\
\text { (dose dependant on SBP, plts, diarrhoea). PGE } 1 \text { was stopped or restarted at a lower dose if } \mathrm{SBP}<100 \\
\text { mmHg, plt }<60,000 \text { or incapacitating diarrhoea } \\
\text { - CMV treatment and prophylaxis regimens (details in article) } \\
\text { - HBV treatment of positive patients. }\end{array}$ \\
\hline Outcomes & $\begin{array}{l}\text { - patient survival } \\
\text { - graft survival } \\
\text { - acute rejection } \\
\text { - steroid resistant rejection } \\
\text { - ALT, AST, ALP, total bilirubin }\end{array}$ \\
\hline
\end{tabular}


Fisher 1998 (Continued)

\begin{tabular}{l|l} 
& - serum creatinine \\
& - haemoglobin, WBC \\
& - hypertension, hypercholesterolaemia, DM, BMI \\
& - conversion \\
& - readmission \\
\hline Notes & Follow-up: initial report 6 months, later report at 4 years. \\
& Correspondence with principle investigator: May 2004 regarding 12 month outcomes \\
Other adverse events (at 6 months): \\
Infections - tacrolimus $(24 / 49) ;$ cyclosporin $(30 / 50)$ \\
CMV - tacrolimus (3/49); cyclosporin $(9 / 50)$.
\end{tabular}

Risk of bias

\begin{tabular}{l|ll}
\hline Item & Authors' judgement & Description \\
\hline Allocation concealment? & Yes & A - Adequate \\
\hline
\end{tabular}

Fung 1991

\begin{tabular}{|c|c|}
\hline Methods & $\begin{array}{l}\text { Generation of the allocation sequence: adequate, computer program implemented the block randomisation } \\
\text { technique. } \\
\text { Allocation concealment: adequate, sealed envelope each containing a single treatment assignment. } \\
\text { Blinding: not performed. } \\
\text { Follow-up: adequate. } \\
\text { Analysis: intention to treat. }\end{array}$ \\
\hline Participants & $\begin{array}{l}\text { Country: USA. } \\
\text { Language: English. } \\
\text { Inclusion criteria: All male and female patients, from } 16 \text { to } 60 \text { years, undergoing primary isolated liver } \\
\text { transplantation. } \\
\text { Exclusion criteria / diagnoses: hepatitis B virus; cancer; infection; advanced renal failure; coma; previous } \\
\text { hepatic surgery; pregnancy or nursing. } \\
\text { Allocation: tacrolimus } \mathrm{n}=41 \text {; cyclosporin } \mathrm{n}=40 \text {. }\end{array}$ \\
\hline Interventions & $\begin{array}{l}\text { Tacrolimus protocol: } \\
\text { - } 0.1 \mathrm{mg} / \mathrm{kg} \text { IV over } 24 \text { hours beginning } 4 \text { hours after the revascularization of the new liver and continued } \\
\text { daily until they were able to take po meds, then } 0.15 \mathrm{mg} / \mathrm{kg} \text { po q } 12 \mathrm{~h} \\
\text { - maintain trough levels from } 1.0 \text { to } 5.0 \mathrm{ng} / \mathrm{ml} \text {. } \\
\text { Cyclosporin protocol: } \\
\text { - cyclosporin formulation: oil-based } \\
\text { - } 4 \mathrm{mg} / \mathrm{kg} \text { IV over } 24 \text { hours, beginning } 4 \text { hours after revascularization until patient was able to take po } \\
\text { meds, then } 8 \mathrm{mg} / \mathrm{kg} \text { po q } 12 \mathrm{~h} \text { to maintain trough level of } 800 \text { to } 1500 \mathrm{ng} / \mathrm{ml} \times 2 \mathrm{months} \text { then decrease } \\
\text { dose to maintain trough of } 600 \text { to } 800 \mathrm{ng} / \mathrm{ml} \text { thereafter: } \\
\text { Concomitant immunosuppression: steroids all patients. } \\
\text {-in both groups, single intra-op } 1 \mathrm{~g} \text { methylprednisolone followed by a daily dose of } 20 \mathrm{mg} / \mathrm{d} \text { until oral } \\
\text { dose was started } \\
\text { - a dose of } 10 \mathrm{mg} / \mathrm{d} \text { was allowed at } 2 \text { weeks if no evidence of rejection }\end{array}$ \\
\hline
\end{tabular}


Fung 1991 (Continued)

\begin{tabular}{ll} 
& - a further reduction to $5 \mathrm{mg} / \mathrm{d}$ was allowed at the end of 1 month if no evidence of rejection \\
& - patients were taken off steroids if there was no evidence of rejection. \\
\hline Outcomes & - patient survival \\
- graft survival & - graft rejection \\
- steroid resistant rejection \\
- conversion \\
- serum creatinine \\
- dialysis \\
- CMV, TB, HCV, HBV, HSV infection \\
- PTLD. \\
\hline Follow-up: 12 months. \\
Correspondence with principle investigator: May 2004 regarding 12 month outcomes. \\
\hline
\end{tabular}

Risk of bias

\begin{tabular}{l|ll}
\hline Item & Author' judgement & Description \\
\hline Allocation concealment? & Yes & A - Adequate \\
\hline
\end{tabular}

\section{Grazi 2004}

\begin{tabular}{|c|c|}
\hline Methods & $\begin{array}{l}\text { Generation of the allocation sequence: adequate, stratified according to hepatitis } \mathrm{C} \text { virus. } \\
\text { Allocation concealment: adequate, central assignment. } \\
\text { Blinding: not performed. } \\
\text { Follow-up: adequate. } \\
\text { Analysis: intention to treat. }\end{array}$ \\
\hline Participants & $\begin{array}{l}\text { Country: Argentina, Australia, Austria, Belgium, Brazil, Canada, France, Germany, Italy, Japan, New } \\
\text { Zealand, Norway, Spain, Sweden, Switzerland, United Kingdom, USA. } \\
\text { Language: English. } \\
\text { Inclusion criteria: All male and female patients, } 18 \text { to } 75 \text { years, undergoing primary isolated liver trans- } \\
\text { plantation. } \\
\text { Exclusion criteria / diagnoses: ABO blood group incompatibility, non-heart-beating donor, cancer, HIV, } \\
\text { unstable fulminant liver failure. } \\
\text { Allocation: cyclosporin } n=250 \text {; tacrolimus } n=245 \text {. }\end{array}$ \\
\hline Interventions & $\begin{array}{l}\text { Tacrolimus protocol: } \\
\text { - started within } 24 \mathrm{hours} \text { of transplant } \\
\text { - from } 0.1 \text { to } 0.15 \mathrm{mg} / \mathrm{kg} / \mathrm{d} \text { in } 2 \text { divided doses po } \\
\text { - after first dose adjusted in target range of } 5 \text { to } 15 \mathrm{ng} / \mathrm{ml} \text { at month } 3 \text { and from } 5 \text { to } 12 \mathrm{ng} / \mathrm{ml} \text { to month } 6 \text {. } \\
\text { Cyclosporin protocol: } \\
\text { - cyclosporin formulation: microemulsion } \\
\text { - started within } 24 \mathrm{hours} \text { of transplant } \\
\text { - from } 10 \text { to } 15 \mathrm{mg} / \mathrm{kg} / \mathrm{d} \text { in } 1 \text { divided doses po } \\
\text { - after first dose adjusted to achieve target range of } 0.8 \text { to } 1.2 \mathrm{ug} / \mathrm{ml} \text { to month } 3 \text { and from } 0.7 \text { to } 0.9 \mathrm{ug} / \mathrm{ml}\end{array}$ \\
\hline
\end{tabular}




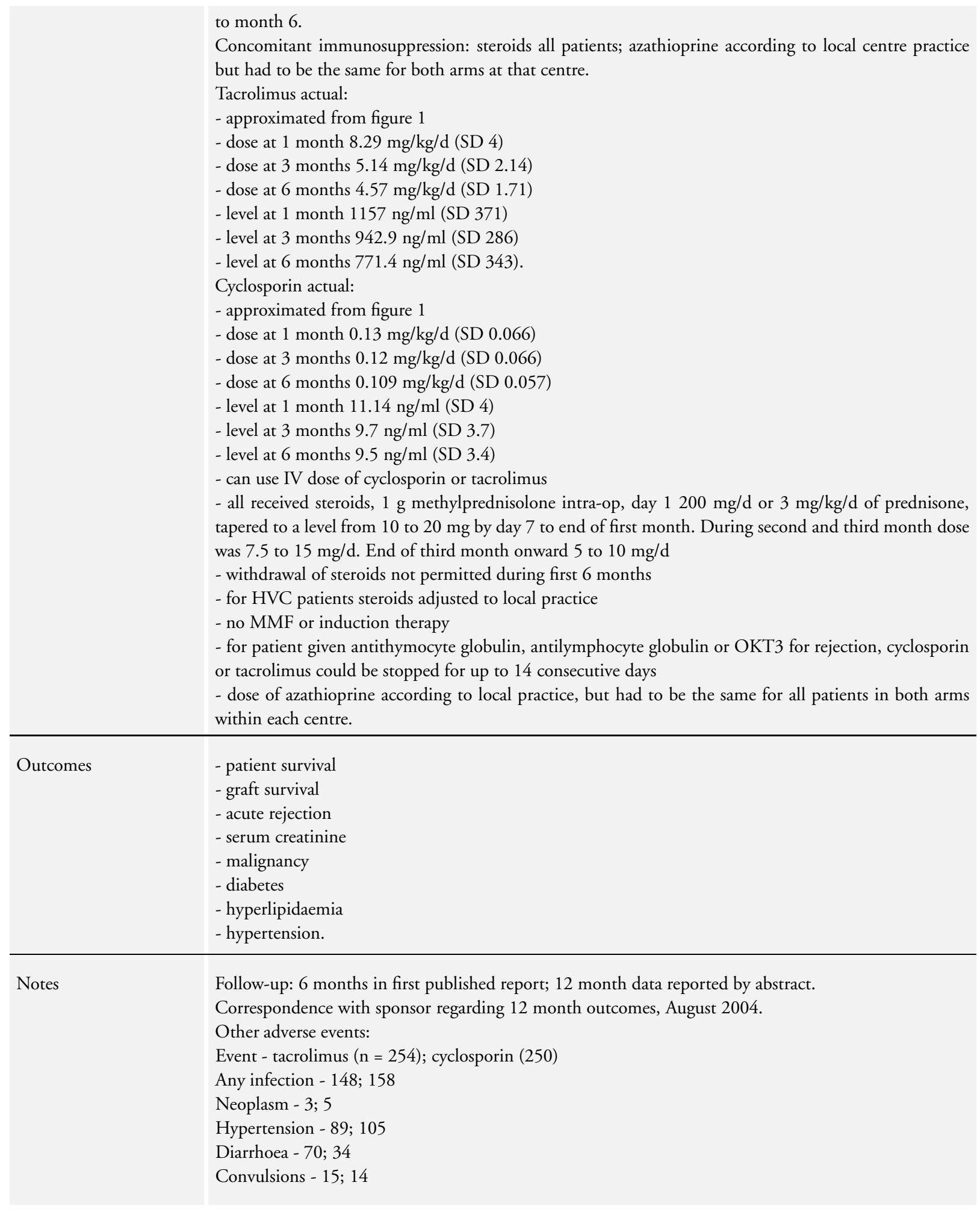


Grazi 2004 (Continued)

Headache $-45 ; 42$

Psychiatric disorders - 121; 109

Hirsutism - 0; 10

Alopecia - 4; 5

Gingival hyperplasia - 0; 5

Pruritus - 19; 13.

\section{Risk of bias}

\begin{tabular}{lll} 
Item & Authors' judgement & Description \\
\hline Allocation concealment? & Yes & A - Adequate \\
\hline
\end{tabular}

\section{Greig 2003}

\begin{tabular}{|c|c|}
\hline Methods & $\begin{array}{l}\text { Generation of the allocation sequence: adequate, stratified by centre. } \\
\text { Allocation concealment: adequate, central assignment. } \\
\text { Blinding: not performed. } \\
\text { Follow-up: adequate. } \\
\text { Analysis: intention to treat. }\end{array}$ \\
\hline Participants & $\begin{array}{l}\text { Country: Canada, multiple centres. } \\
\text { Language: English. } \\
\text { Inclusion criteria: All male and female patients, over } 16 \text { years, undergoing primary isolated liver trans- } \\
\text { plantation. } \\
\text { Exclusion criteria / diagnoses: ABO blood group incompatibility, advanced renal failure, cancer, acute } \\
\text { pancreatitis, life expectancy less than } 2 \text { weeks. } \\
\text { Allocation: tacrolimus } n=71 \text {; cyclosporin } n=72 \text {. }\end{array}$ \\
\hline Interventions & $\begin{array}{l}\text { Tacrolimus protocol: } \\
\text { - from } 0.1 \text { to } 0.15 \mathrm{mg} / \mathrm{kg} / \mathrm{d} \text { divided twice daily NG/po to maintain target trough of } 10 \text { to } 20 \mathrm{ng} / \mathrm{ml} \text { during } \\
1 \text { st month and from } 5 \text { to } 15 \mathrm{ng} / \mathrm{ml} \text { thereafter } \\
\text { Cyclosporin protocol: } \\
\text { - cyclosporin formulation: microemulsion } \\
\text { - from } 10 \text { to } 15 \mathrm{mg} / \mathrm{kg} / \mathrm{d} \text { divided twice daily NG/po to maintain target trough of } 300 \text { to } 400 \mathrm{ng} / \mathrm{ml} \text { days } \\
1 \text { to } 14 \text {, from } 250 \text { to } 350 \mathrm{ng} / \mathrm{ml} \text { days } 15 \text { to } 28 \text {, from } 200 \text { to } 300 \mathrm{ng} / \mathrm{ml} \text { days } 29 \text { to } 90 \text { and from } 100-250 \\
\text { ng/ml thereafter: } \\
\text { Concomitant immunosuppression: steroids all patients; azathioprine all patients. } \\
\text { Tacrolimus actual: } \\
\text { - approximated from Figure } 2 \\
\text { - dose at } 1 \text { month } 14 \mathrm{mg}(\mathrm{SD} 6.5) \\
\text { - dose at } 3 \text { months } 11 \mathrm{mg}(\mathrm{SD} 6.0) \\
\text { - dose at } 6 \text { months } 9 \mathrm{mg}(\mathrm{SD} 5.5) \\
\text { - dose at } 12 \text { months } 7 \mathrm{mg}(\mathrm{SD} 5.0) \\
\text { - trough at } 1 \text { month } 12.5 \mathrm{ng} \cdot \mathrm{ml} \\
\text { - trough at } 3 \text { months } 11.75 \mathrm{ng} / \mathrm{ml} \\
\text { - trough at } 6 \text { months } 11.75 \mathrm{mg} / \mathrm{ml} \\
\text { - trough at } 12 \text { months } 10 \mathrm{ng} / \mathrm{ml} \text {. }\end{array}$ \\
\hline
\end{tabular}


Greig 2003 (Continued)

\begin{tabular}{|c|c|}
\hline & 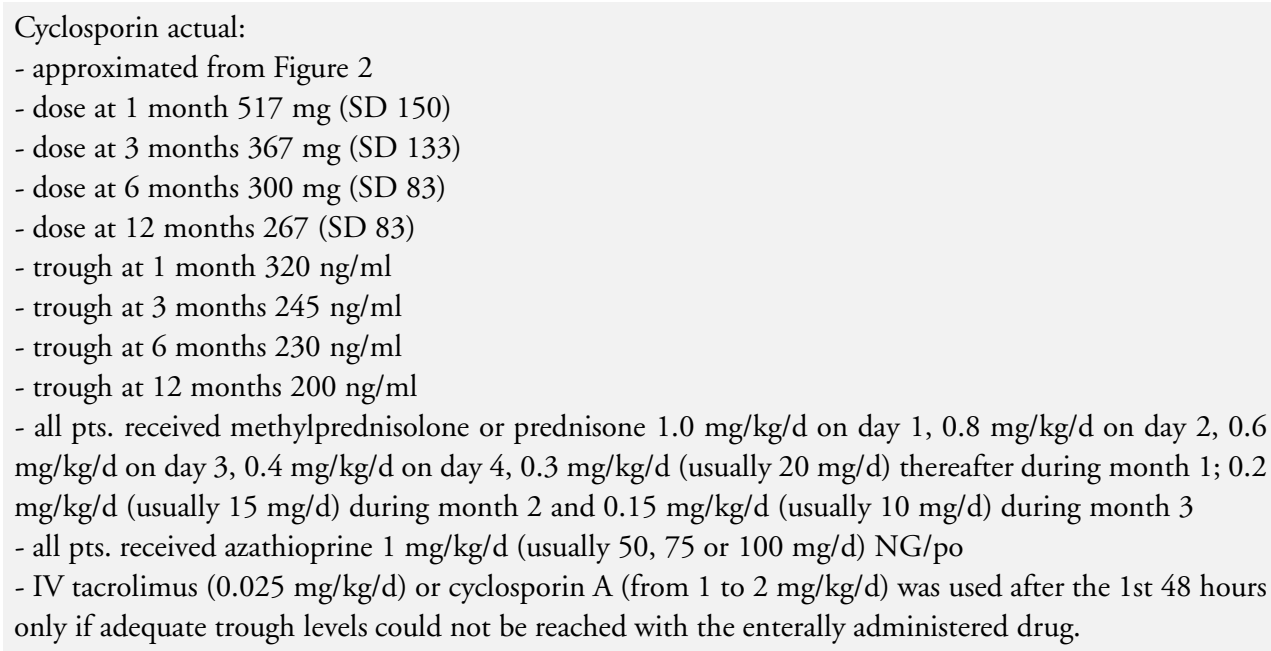 \\
\hline Outcomes & $\begin{array}{l}\text { - patient survival } \\
\text { - graft survival } \\
\text { - acute rejection } \\
\text { - steroid resistant rejection } \\
\text { - steroid withdrawal } \\
\text { - dialysis } \\
\text { - diabetes } \\
\text { - creatinine } \\
\text { - PTLD } \\
\text { - coma, seizures, confusion, delirium, hallucinations, psychosis, depression, anxiety/nervousness, insom- } \\
\text { nia, somnolence, tremor, headache } \\
\text { - viral, bacterial and fungal infection. }\end{array}$ \\
\hline Notes & $\begin{array}{l}\text { Follow-up: } 12 \text { months. } \\
\text { Correspondence with sponsor: June } 2004 \text { regarding } 12 \text { month outcomes. } \\
\text { Other adverse events (only \% reported): } \\
\text { Events - tacrolimus; cyclosporin } \\
\text { Coma - 0; } 7 \% \\
\text { Seizures - 3\%; 3\% } \\
\text { Confusion - 23\%; 30\% } \\
\text { Delirium - 4\%; 7\% } \\
\text { Hallucinations - 14\%; } 13 \% \\
\text { Psychosis - } 1 \% ; 1 \% \\
\text { Depression - 24\%; } 18 \% \\
\text { Anxiety - } 63 \% ; 48 \% \\
\text { Insomnia - } 66 \% ; 46 \% \\
\text { Somnolence - } 10 \% ; 13 \% \\
\text { Tremor - } 63 \% ; 57 \% \\
\text { Headache - } 75 \% ; 64 \% \\
\text { Viral infections - } 61 \% ; 49 \% \\
\text { Bacterial infections - } 76 \% ; 69 \% \\
\text { Fungal infections - } 24 \% ; 25 \% \text {. }\end{array}$ \\
\hline
\end{tabular}


Greig 2003 (Continued)

\section{Risk of bias}

\begin{tabular}{l|ll}
\hline Item & Authors' judgement & Description \\
\hline Allocation concealment? & Yes & A - Adequate \\
\hline
\end{tabular}

Kelly 2004

Methods

Gethods
treatment centre.
Allocation concealment: adequate, central assignment.
Blinding: not performed.
Follow-up: adequate.
Analysis: intention to treat.

Participants

Country: Europe, multiple centres.
Language: English.

Inclusion criteria: All male and female patients, 16 years or younger, undergoing primary isolated liver transplantation.

Exclusion criteria / diagnoses: ABO blood group incompatibility, infections, HIV, cancer or history of cancer, history of sensitivity to test agents.

Allocation: tacrolimus $\mathrm{n}=91$; cyclosporin $\mathrm{n}=90$.

Interventions

First dose was to be given within 6 hours of transplantation.

Tacrolimus protocol:

- tacrolimus $0.3 \mathrm{mg} / \mathrm{kg}$ per day (po,NG, NJ) in two divided doses (doses adjusted to maintain trough of 10 to $20 \mathrm{mg} / \mathrm{L}$ in first 2 weeks, from 10 to $15 \mathrm{mg} / \mathrm{L}$ during weeks 3 to 4 , from 5 to $15 \mathrm{mg} / \mathrm{L}$ during months 2 to 3 and from 5 to $10 \mathrm{mg} / \mathrm{L}$ thereafter)

Cyclosporin protocol:

- cyclosporin formulation: microemulsion

- $10 \mathrm{mg} / \mathrm{kg} / \mathrm{d}$ given in two divided doses (rest of doses adjusted to maintain trough of $250-350 \mathrm{mg} / \mathrm{L}$ within first 2 weeks, from $200-300 \mathrm{mg} / \mathrm{L}$ during weeks 3 to 12 , from $150-200 \mathrm{mg} / \mathrm{L}$ during months 4 to 12 and from $100-150 \mathrm{mg} / \mathrm{L}$ thereafter.

Concomitant immunosuppression: steroids all patients; azathioprine to cyclosporin recipients according to local practice.

Tacrolimus actual:

- mean dose $0.28 \mathrm{mg} / \mathrm{kg}$ (SD 0.16) for month $1,0.18 \mathrm{mg} / \mathrm{kg}$ (SD 0.11) months 10 to 12

- mean trough $13.9 \mathrm{mg} / \mathrm{L}$ (SD 3.1) month $1,7.3 \mathrm{mg} / \mathrm{L}$ (SD 2.8 for months 10 to12.

Cyclosporin actual:

- mean dose $16.17 \mathrm{mg} / \mathrm{kg}$ (SD 9.77) month $1,8.86 \mathrm{mg} / \mathrm{kg}$ (SD 2.96) months 10 to 12

- mean trough $263.4 \mathrm{mg} / \mathrm{l}$ (SD 81.5) month 1, $144.0 \mathrm{mg} / \mathrm{L}$ (SD 34.9) months 10 to 12

- can obtain approximate 3 and 6 month data from figure 2

- both treatment groups received: IV methyl prednisolone $10 \mathrm{mg} / \mathrm{kg}$ intraop and $2 \mathrm{mg} / \mathrm{kg}$ per day days 1 to 6

- oral prednisolone was given at daily does of $1 \mathrm{mg} / \mathrm{kg}$ at days $7-13,0.75 \mathrm{mg} / \mathrm{kg}$ at days 14 to $20,0.5$ $\mathrm{mg} / \mathrm{kg}$ at days 21 to 28 and $0.25 \mathrm{mg} / \mathrm{kg}$ at months 2 to 3 . Thereafter, steroids could be tapered off in accordance with centres protocol 
Kelly 2004 (Continued)

- patients in cyclosporin group received azathioprine at $1.5 \mathrm{mg} / \mathrm{kg} / \mathrm{d}$ for the first 3 months, thereafter, discontinuation was optional.

\begin{tabular}{|c|c|}
\hline Outcomes & $\begin{array}{l}\text { - patient survival } \\
\text { - graft survival } \\
\text { - acute rejection } \\
\text { - steroid resistant rejection } \\
\text { - creatinine }\end{array}$ \\
\hline Notes & $\begin{array}{l}\text { Follow-up: } 12 \text { months. } \\
\text { Other adverse events: } \\
\text { Event - tacrolimus ( } \mathrm{n}=91) \text {; cyclosporin }(\mathrm{n}=90) \\
\text { Fever - 42; } 46 \\
\text { Hypomagnesemia - 36; } 26 \\
\text { Hypertension - 35; } 42 \\
\text { Abnormal liver function tests - 34, } 25 \\
\text { Anaemia - 26; } 17 \\
\text { Diarrhoea - 24; } 23 \\
\text { Acidosis - 24; } 15 \\
\text { Sepsis - 20; } 18 \\
\text { Pleural effusion - 20; } 17 \\
\text { Ascites - 15; } 18 \\
\text { Hirsutism - 1; } 25 \\
\text { Gingival hypertrophy - } 1 ; 8 \\
\text { CMV infection - } 14 ; 22 \\
\text { EBV infection - 24; } 10 .\end{array}$ \\
\hline
\end{tabular}

\section{Risk of bias}

\begin{tabular}{lll}
\hline Item & Authors' judgement & Description \\
\hline Allocation concealment? & Yes & A - Adequate \\
\hline
\end{tabular}

\section{Klupp 1999}

\begin{tabular}{ll} 
Methods & Generation of the allocation sequence: adequate, three-arm trial. \\
& Allocation concealment: adequate, sealed envelope. \\
Blinding: not performed. & Follow-up: adequate. \\
& Analysis: intention to treat. \\
\hline Participants & $\begin{array}{l}\text { Country: Germany, single centre. } \\
\text { Language: English. } \\
\text { Inclusion criteria: Male and female patients undergoing primary isolated liver transplantation. } \\
\text { Exclusion criteria / diagnoses: not specified. } \\
\text { Allocation: cyclosporin/MMF } \mathrm{n}=40 ; \text { tacrolimus/MMF } \mathrm{n}=40 ; \text { tacrolimus } \mathrm{n}=40 .\end{array}$
\end{tabular}




\section{Klupp 1999 (Continued)}

\begin{tabular}{l|l} 
Interventions & Concomitant immunosuppression: steroids all patients; MMF all patients. \\
& Dosages of tacrolimus, cyclosporin and MMF according to usual protocol of centre, not given in report. \\
All patients received low dose prednisone. & Cyclosporin formulation: microemulsion \\
\hline Outcomes & - patient survival \\
- graft survival & - acute rejection \\
- steroid resistant rejection \\
- OKT3 rescue therapy \\
- mean bilirubin \\
- bile production \\
- bacterial, CMV and fungal infection \\
- switching from cyclosporin to tacrolimus and vice versa \\
\hline Follow up: 12 to 26 month outcomes reported. 12 month data supplied by principal investigator in April \\
2005. \\
Other adverse events: \\
Bacterial infections - tacrolimus (14/40); cyclosporin (20/40) \\
CMV - tacrolimus (13/40); cyclosporin (11/40) \\
Fungal infections - tacrolimus (8/20); cyclosporin (7/40).
\end{tabular}

\section{Risk of bias}

\begin{tabular}{l|ll}
\hline Item & Authors' judgement & Description \\
\hline Allocation concealment? & Yes & A - Adequate \\
\hline
\end{tabular}

\section{Martin 2004}

Methods

Generation of the allocation sequence: adequate, stratified by centre.

Allocation concealment: adequate, central randomisation.

Blinding: not performed.

Follow-up: adequate.

Analysis: intention to treat.

$\begin{array}{ll}\text { Participants } & \text { Country: USA, multiple centres. } \\ & \text { Language: English. } \\ & \text { Inclusion criteria: All male and female patients with hepatitis C virus, } 18 \text { years or older, undergoing } \\ \text { primary isolated liver transplantation. } \\ \text { Exclusion criteria / diagnoses: } \mathrm{ABO} \text { blood group incompatibility, Hepatitis B virus, HIV, cancer, preg- } \\ \text { nancy, lactation. } \\ \text { Allocation: tacrolimus } \mathrm{n}=38 \text {; cyclosporin } \mathrm{n}=41 .\end{array}$


Martin 2004 (Continued)

\begin{tabular}{l|l} 
& - cyclosporin formulation: microemulsion \\
& - started 12 hours after transplantation; from 6 to $10 \mathrm{mg} / \mathrm{kg} / \mathrm{d}$ adjusted to maintain trough of 200 to 250 \\
& ng/ml for first 6 months and $100 \mathrm{to} 250 \mathrm{ng} / \mathrm{ml}$ for next 6 months: \\
& $\begin{array}{l}\text { Concomitant immunosuppression: steroids all patients; azathioprine all patients. } \\
\text { - all patients received periop parenteral steroids which were tapered to } 20 \mathrm{mg} / \mathrm{d} \text { orally on day } 6 \text { and } \\
\text { decreased to } 5 \mathrm{mg} / \mathrm{d} \text { at day } 90 \\
\text { - all patients received azathioprine - } 2 \mathrm{mg} / \mathrm{kg} / \mathrm{d} \text { tapered to } 1 \mathrm{mg} / \mathrm{kg} / \mathrm{d} \text { by day } 7 \text { and then withdrawn } \\
\text { gradually after } 60 \text { days } \\
\text { - no OKT3 was used for rejection. }\end{array}$ \\
\hline Outcomes & $\begin{array}{l}\text { - recurrence of histologically diagnosed hepatitis } \\
\text { - time to hepatitis C virus recurrence } \\
\text { - change in viral load } \\
\text { - graft survival } \\
\text { - patient survival } \\
\text { - biopsy-proven rejection rate. }\end{array}$ \\
\hline Notes & \begin{tabular}{l} 
Follow-up: 12 months. \\
\hline Risk of bias
\end{tabular} \\
\hline Item & Authors judgement \\
\hline Allocation concealment? & Yes \\
\hline
\end{tabular}

Muehlbacher 2001

\begin{tabular}{ll} 
Methods & Generation of the allocation sequence: adequate, stratified by centre. \\
Allocation concealment: adequate, central randomisation. \\
Blinding: not performed. \\
Follow-up: adequate. \\
Analysis: intention to treat. \\
\hline Participants \\
$\begin{array}{l}\text { Country: Europe, multiple centres. } \\
\text { Language: English. } \\
\text { Inclusion criteria: Adult patients, } 18 \text { years or older, undergoing primary isolated liver transplantation. } \\
\text { Exclusion criteria / diagnoses: pregnancy, intolerance of test agents, uncontrolled infection, HIV, extra- } \\
\text { hepatic malignancy. } \\
\text { Allocation: tacrolimus } \mathrm{n}=310 ; \text { cyclosporin } \mathrm{n}=305 .\end{array}$ \\
$\begin{array}{l}\text { Tacrolimus protocol: } \\
\text { - } 0.15 \mathrm{mg} / \mathrm{kg} / \mathrm{d} \text { adjusted to achieve trough of } 10 \text { to } 20 \mathrm{ng} / \mathrm{mL} \text { for the } 1 \text { st month and from } 5 \text { to } 15 \mathrm{ng} / \mathrm{mL} \\
\text { thereafter: } \\
\text { Cyclosporin protocol: } \\
\text { - cyclosporin formulation: microemulsion } \\
\text { - from } 8 \text { to } 15 \mathrm{mg} / \mathrm{kg} \text { adjusted to achieve trough } 150-300 \mathrm{ng} / \mathrm{mL} \text { throughout study. } \\
\text { Concomitant immunosuppression: steroids to all patients; azathioprine to cyclosporin recipients according } \\
\text { to local practice. }\end{array}$
\end{tabular}


Muehlbacher 2001 (Continued)

\begin{tabular}{|c|c|c|}
\hline & \multicolumn{2}{|c|}{$\begin{array}{l}\text { Tacrolimus actual: } \\
\text { - mean dose at } 1 \text { year } 0.07(+/-0.045) \mathrm{mg} / \mathrm{kg} \\
\text { - mean trough levels at } 1 \text { year } 9.15(+/-3.42) \mathrm{ng} / \mathrm{mL} \text {. } \\
\text { Cyclosporin actual: } \\
\text { - mean dose at } 1 \text { year } 3.3(+/-1.29) \mathrm{mg} / \mathrm{kg} \\
\text { - mean trough at } 1 \text { year } 170(+/-63) \mathrm{ng} / \mathrm{ml} \\
\text { - tapering steroid scheme } \\
\text { - } 43 \% \text { were off steroids in either group at } 1 \text { year. }\end{array}$} \\
\hline Outcomes & \multicolumn{2}{|l|}{$\begin{array}{l}\text { - patient survival } \\
\text { - graft survival } \\
\text { - acute rejection } \\
\text { - steroid resistant rejection } \\
\text { - hypertension } \\
\text { - need for oral antidiabetic drugs } \\
\text { - use of insulin. }\end{array}$} \\
\hline Notes & \multicolumn{2}{|c|}{$\begin{array}{l}\text { Follow-up: } 3 \text { months in first report and } 12 \text { months in abstract. } \\
\text { Correspondence with sponsor regarding protocol, } 12 \text { month data in June } 2004 . \\
\text { Other adverse events (only \% reported, only } 3 \text { month follow-up): } \\
\text { Event - tacrolimus; cyclosporin } \\
\text { Diarrhoea - } 12.9 \% ; 4.9 \% \\
\text { Biliary system abnormalities }-5.2 \% ; 9.5 \% \\
\text { Hyperuricaemia - } 1.3 \% ; 3.9 \% \text {. }\end{array}$} \\
\hline \multicolumn{3}{|l|}{ Risk of bias } \\
\hline Item & Authors' judgement & Description \\
\hline Allocation concealment? & Yes & A - Adequate \\
\hline
\end{tabular}

\section{O’Grady 2002}

Methods

Generation of the allocation sequence: adequate, stratified and blocked randomised sequences using computer-generated random numbers. Allocation was stratified for emergency and elective transplantation within each centre: randomisation for elective treatment had an equal number of blocks of size 6,8 and 10 and ER treatment had an equal number of blocks of size 4 and 6; blocks were ordered at random. Allocation concealment: adequate, serially numbered opaque envelopes.

Blinding: not performed.

Follow-up: adequate.

Analysis: intention to treat.

Participants

Country: UK, multiple centres.

Language: English.

Inclusion criteria: All male and female patients, 18 years or older, undergoing primary isolated liver transplantation.

Exclusion criteria / diagnoses: $\mathrm{ABO}$ blood group incompatibility, pregnancy, lactation, contraindication to test agents. 
O’Grady 2002 (Continued)

Allocation: tacrolimus $\mathrm{n}=301$; cyclosporin $\mathrm{n}=305$.

\begin{tabular}{|c|c|}
\hline Interventions & $\begin{array}{l}\text { Preferred assignment within } 6 \text { hours of transplantation; initial dose po/NG/NJ within } 48 \text { hours of trans- } \\
\text { plant. } \\
\text { Tacrolimus protocol: } \\
\text { - } 0.1 \mathrm{mg} / \mathrm{kg} / \mathrm{d} \text { up to a maximum of } 10 \mathrm{mg} / \mathrm{d} \text { to maintain target trough level of } 5 \text { to } 15 \mathrm{ug} / \mathrm{L} \text {. } \\
\text { Cyclosporin protocol: } \\
\text { - cyclosporin formulation: microemulsion } \\
\text { - } 10 \mathrm{mg} / \mathrm{kg} / \mathrm{d} \text { divided q } 12 \mathrm{~h} \text { to maintain a target trough level of } 200 \text { to } 300 \mathrm{ug} / \mathrm{ml} \text { within the } 1 \mathrm{st} \text { month } \\
\text { and from } 150 \text { to } 250 \mathrm{ug} / \mathrm{ml} \text { thereafter } \\
\text { - prednisone in both groups tapering dose starting at } 20 \mathrm{mg} / \text { day reducing to } 7.5 \mathrm{mg} / \text { day at } 3 \text { months or } \\
\text { equivalent dose of methylprednisolone or hydrocortisone } \\
\text { - azathioprine in both groups at } 1 \mathrm{mg} / \mathrm{kg} / \text { day } \mathrm{IV} / \text { po. }\end{array}$ \\
\hline Outcomes & $\begin{array}{l}\text { - patient survival } \\
\text { - graft survival } \\
\text { - graft rejection } \\
\text { - diabetes } \\
\text { - renal function } \\
\text { - neurological complications } \\
\text { - body hair abnormalities } \\
\text { - malignant disease }\end{array}$ \\
\hline Notes & $\begin{array}{l}\text { Follow-up: } 12 \text { months. } \\
\text { Other adverse events: } \\
\text { Event - tacrolimus ( } \mathrm{n}=301) \text {; cyclosporin }(\mathrm{n}=306) \\
\text { Infection treated - 265; } 263 \\
\text { Seizures - 17; } 11 \\
\text { Neuropathy (by nerve conduction studies) - } 3 ; 3 \\
\text { Neuropathy (subjective)- } 31 ; 27 \\
\text { Psychosis - 20; } 22 \\
\text { Coma - 9; } 8 \\
\text { Headaches - 56; } 52 \\
\text { Pruritus - 33; } 25 \\
\text { Hypertension treated - 56; } 70 \\
\text { Hypertrichosis - 9;25 } \\
\text { Alopecia - 20; } 7 .\end{array}$ \\
\hline
\end{tabular}

Risk of bias

\begin{tabular}{l|ll}
\hline Item & Authors' judgement & Description \\
\hline Allocation concealment? & Yes & A - Adequate \\
\hline
\end{tabular}


Rolles 1999

\begin{tabular}{|c|c|}
\hline Methods & $\begin{array}{l}\text { Generation of the allocation sequence: not clear. } \\
\text { Allocation concealment: not clear. } \\
\text { Blinding: not performed. } \\
\text { Follow-up: adequate. } \\
\text { Analysis: intention to treat. }\end{array}$ \\
\hline Participants & $\begin{array}{l}\text { Country: UK, single centre. } \\
\text { Language: English. } \\
\text { Inclusion criteria: Adult patients undergoing primary isolated liver transplantation. } \\
\text { Exclusion criteria / diagnoses: not specified. } \\
\text { Allocation: tacrolimus } n=30 \text {; cyclosporin } n=34 \text {. }\end{array}$ \\
\hline Interventions & 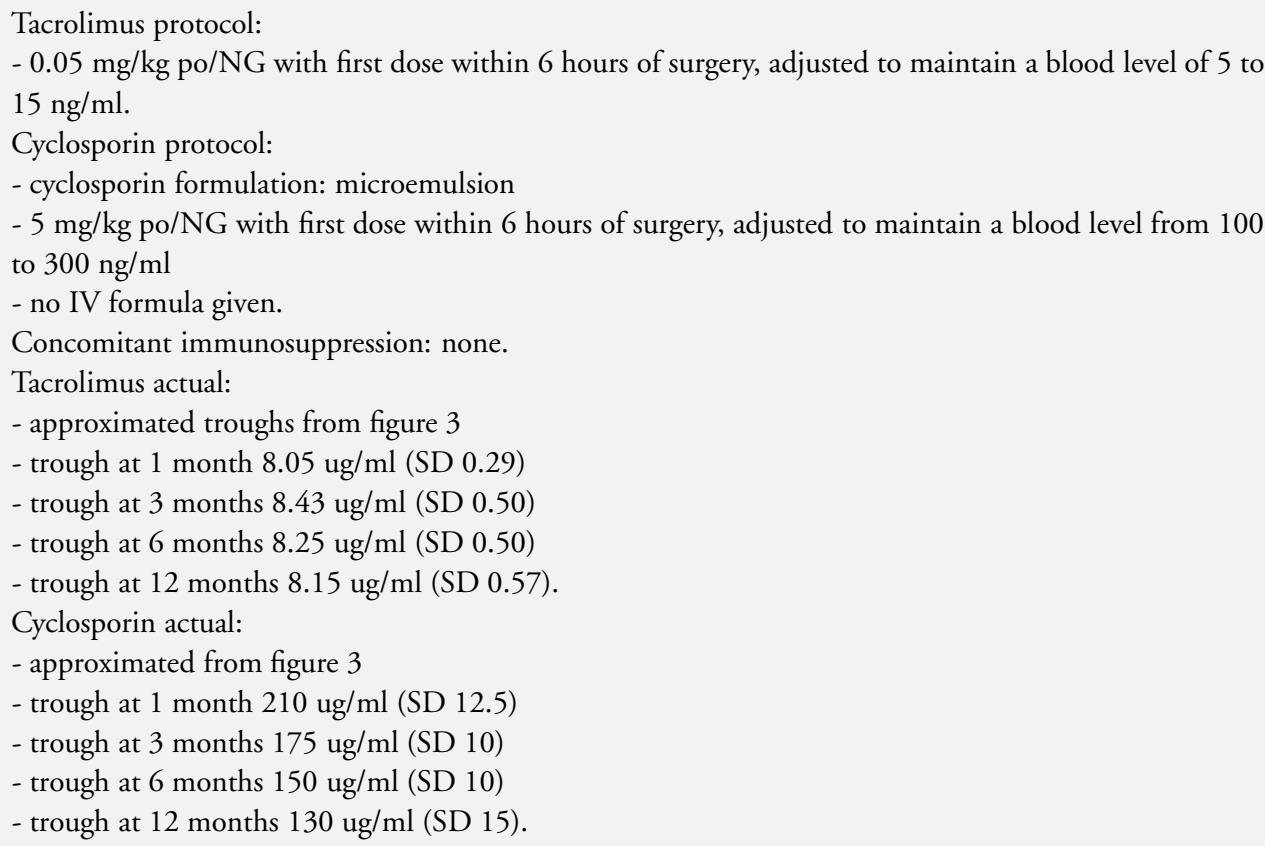 \\
\hline Outcomes & $\begin{array}{l}\text { - patient survival } \\
\text { - graft survival } \\
\text { - acute rejection }\end{array}$ \\
\hline es & 2 months. \\
\hline
\end{tabular}

\section{Risk of bias}

\begin{tabular}{l|ll}
\hline Item & Authors' judgement & Description \\
\hline Allocation concealment? & Unclear & B - Unclear \\
\hline
\end{tabular}




\begin{tabular}{|c|c|}
\hline Methods & $\begin{array}{l}\text { Generation of the allocation sequence: not clear. } \\
\text { Allocation concealment: not clear. } \\
\text { Blinding: not performed. } \\
\text { Follow-up: adequate. } \\
\text { Analysis: intention to treat. }\end{array}$ \\
\hline Participants & $\begin{array}{l}\text { Country: USA, single centre. } \\
\text { Language: English. } \\
\text { Inclusion criteria: All male and female patients, } 18 \text { years or older, undergoing orthotopic cadaver liver } \\
\text { transplantation. } \\
\text { Exclusion criteria / diagnoses: hepatitis B virus. } \\
\text { Allocation: tacrolimus } \mathrm{n}=35 \text {; cyclosporin } \mathrm{n}=36 \text {. }\end{array}$ \\
\hline Interventions & $\begin{array}{l}\text { Tacrolimus protocol: } \\
\text { - } 6 \mathrm{mg} / \text { day po/NG adjusted to maintain a blood level of } 10 \text { to } 15 \mathrm{ng} / \mathrm{ml} \text {. } \\
\text { Cyclosporin protocol: } \\
\text { - cyclosporin formulation: microemulsion } \\
\text { - } 600 \mathrm{mg} / \text { day po/NG adjusted to maintain a blood level from } 300 \text { to } 350 \mathrm{ng} / \mathrm{ml} \\
\text { - no IV formula given. } \\
\text { Concomitant immunosuppression: steroids all patients; MMF all patients. Prednisone was withdrawn at } \\
2 \text { weeks. }\end{array}$ \\
\hline Outcomes & $\begin{array}{l}\text { - patient survival } \\
\text { - graft survival } \\
\text { - acute rejection. }\end{array}$ \\
\hline Notes & $\begin{array}{l}\text { Follow-up: results only available at } 6 \text { months even after correspondence with principle investigator. } \\
\text { Other adverse events: } \\
\text { Hypertension - tacrolimus }(3 / 25) \text {; cyclosporin }(10 / 33) \\
\text { CMV - tacrolimus (2/25); cyclosporin }(2 / 33) \text {. }\end{array}$ \\
\hline
\end{tabular}

\section{Risk of bias}

\begin{tabular}{l|ll}
\hline Item & Authors' judgement & Description \\
\hline Allocation concealment? & Unclear & B - Unclear \\
\hline
\end{tabular}

\section{Therapondos 2002}

\begin{tabular}{ll}
\hline Methods & Generation of the allocation sequence: adequate. Computer generated. \\
& $\begin{array}{l}\text { Allocation concealment: adequate. Numbered envelopes. } \\
\text { Blinding: not performed. } \\
\text { Follow-up: adequate. } \\
\text { Analysis: intention to treat. }\end{array}$ \\
\hline Participants & $\begin{array}{l}\text { Country: Scotland, single centre. } \\
\text { Language: English. } \\
\text { Inclusion criteria: Adult patients undergoing primary isolated liver transplantation. }\end{array}$ \\
\hline
\end{tabular}


Therapondos 2002 (Continued)

Exclusion criteria / diagnoses: not specified.

Allocation: tacrolimus $\mathrm{n}=20$; cyclosporin $\mathrm{n}=20$.

\begin{tabular}{|c|c|c|}
\hline Interventions & \multicolumn{2}{|c|}{$\begin{array}{l}\text { Tacrolimus protocol: } \\
\text { - oral/NG within } 6 \text { hours of transplant } \\
\text { - } 0.1 \mathrm{mg} / \mathrm{kg} \text {, target level from } 5 \text { to } 15 \mathrm{ng} / \mathrm{ml} \text {. } \\
\text { Cyclosporin protocol: } \\
\text { - cyclosporin formulation: microemulsion } \\
\text { - oral } / \mathrm{NG} \text { within } 6 \text { hours of transplant } \\
\text { - } 10 \mathrm{mg} / \mathrm{kg} \text {, target level from } 150 \text { to } 200 \mathrm{nmol} / \mathrm{L} \\
\text { Concomitant immunosuppression: steroids all patients; azathioprine all patients. } \\
\text { Azathioprine } 2 \mathrm{mg} / \mathrm{kg} \text { and prednisolone } 20 \mathrm{mg} \times 4 \text { weeks, reduced by } 5 \mathrm{mg} \text { per subsequent month }\end{array}$} \\
\hline Outcomes & \multicolumn{2}{|l|}{$\begin{array}{l}\text { - patient survival } \\
\text { - cardiac events } \\
\text { - withdrawals } \\
\text { - blood pressure } \\
\text { - ECG changes } \\
\text { - echocardiographic data } \\
\text { - brain natriuretic peptide levels } \\
\text { - HRV (?) } \\
\text { - nutritional parameters. }\end{array}$} \\
\hline Notes & \multicolumn{2}{|c|}{$\begin{array}{l}\text { Follow-up: } 12 \text { month data provided by principal investigator (April 2005). } \\
\text { Other adverse events: } \\
\text { Cardiac events - tacrolimus (4/20); cyclosporin ( } 4 / 20) \text {. }\end{array}$} \\
\hline \multicolumn{3}{|l|}{ Risk of bias } \\
\hline Item & Authors' judgement & Description \\
\hline Allocation concealment? & Yes & A - Adequate \\
\hline
\end{tabular}

Timmermann 2002

Methods

Generation of the allocation sequence: adequate. Random numbers.

Allocation concealment: adequate. Third party allocation.

Blinding: not performed.

Follow-up: adequate.

Analysis: intention to treat.

Participants

Country: Germany, multiple centres.

Language: English.

Inclusion criteria: Adult patients undergoing primary isolated liver transplantation.

Exclusion criteria / diagnoses: not specified.

Allocation: tacrolimus $n=72$; cyclosporin $n=71$. 
Timmermann 2002 (Continued)

\begin{tabular}{|c|c|c|}
\hline Interventions & \multicolumn{2}{|c|}{$\begin{array}{l}\text { Tacrolimus protocol: } \\
\text { - oral/NG within } 6 \text { hours of transplant } \\
\text { - } 0.1 \mathrm{mg} / \mathrm{kg} \text {, target level from } 5 \text { to } 15 \mathrm{ng} / \mathrm{ml} \text {. } \\
\text { Cyclosporin protocol: } \\
\text { - cyclosporin formulation: microemulsion } \\
\text { - oral/NG within } 6 \text { hours of transplant dosed according to local practice. } \\
\text { Concomitant immunosuppression: steroids and antilymphocyte globulin given to each group according } \\
\text { to local practice; azathioprine given to cyclosporin patients according to local practice. }\end{array}$} \\
\hline Outcomes & \multicolumn{2}{|l|}{$\begin{array}{l}\text { - patient survival } \\
\text { - patient survival } \\
\text { - acute rejection } \\
\text { - diabetes. }\end{array}$} \\
\hline Notes & \multicolumn{2}{|c|}{$\begin{array}{l}\text { Study only maintained for } 3 \text { months. Data for later follow up not available, confirmed by sponsor (April } \\
2005) \text {. } \\
\text { Other adverse events (only \% reported): } \\
\text { Event - tacrolimus; cyclosporin } \\
\text { Infections - } 21.5 \% ; 26.1 \% \\
\text { CMV - } 15.4 \% ; 17.4 \% \\
\text { hypertension - } 13.8 \% ; 20.3 \% \\
\text { tremor - } 6.2 \% ; 0 .\end{array}$} \\
\hline \multicolumn{3}{|l|}{ Risk of bias } \\
\hline Item & Authors' judgement & Description \\
\hline Allocation concealment? & Yes & A - Adequate \\
\hline
\end{tabular}

\section{U. S. Study 1994}

\begin{tabular}{ll} 
Methods & $\begin{array}{l}\text { Generation of the allocation sequence: adequate, centrally generated by computer, randomly assigned in } \\
\text { blocks of four. } \\
\text { Allocation concealment: adequate. Numbered envelopes. } \\
\text { Blinding: not performed. } \\
\text { Follow-up: adequate. } \\
\text { Analysis: intention to treat. }\end{array}$ \\
\hline Participants & $\begin{array}{l}\text { Country: Eight centres in four European countries. } \\
\text { Language: English. } \\
\text { Inclusion criteria: Male and female patients, all ages, undergoing primary isolated liver transplantation. } \\
\text { Exclusion criteria / diagnoses: vasculitis, cancer, renal failure, HIV, coma, pregnancy, treatment with } \\
\text { anticoagulants, ABO blood group incompatibility. } \\
\text { Allocation: tacrolimus } \mathrm{n}=263 ; \text { cyclosporin } \mathrm{n}=266 .\end{array}$ \\
\hline $\begin{array}{l}\text { Tacrolimus protocol: } \\
\text { - initial } 0.075 \mathrm{mg} / \mathrm{kg} \text { in a } 4 \text { hours IV infusion every } 12 \text { hours until it could be taken orally } \\
\text { - IV was reduced to } 0.05 \mathrm{mg} / \mathrm{kg} \text { over a } 12 \text { hour period twice daily after first } 48 \text { patients (discovered renal }\end{array}$ \\
\hline
\end{tabular}




\begin{tabular}{|c|c|}
\hline & 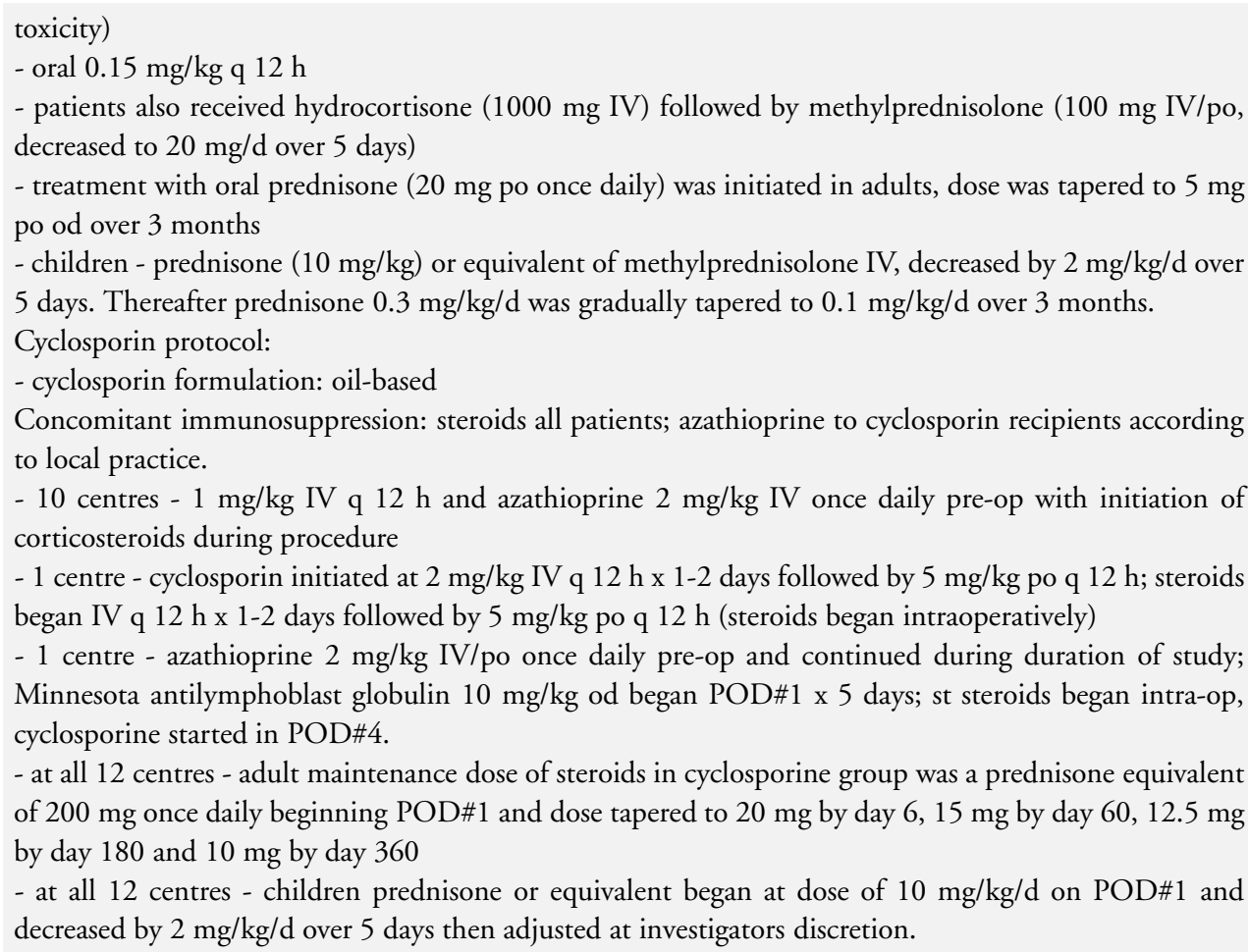 \\
\hline Outcomes & $\begin{array}{l}\text { - patient survival } \\
\text { - graft survival } \\
\text { - LOS in hospital } \\
\text { - acute rejection } \\
\text { - steroid resistant rejection } \\
\text { - withdrawal } \\
\text { - creatinine } \\
\text { - mean GFR } \\
\text { - de novo diabetes } \\
\text { - de novo dialysis } \\
\text { - PTLD. }\end{array}$ \\
\hline
\end{tabular}

Notes

Follow-up: 12 months.

Other adverse events:

Event - tacrolimus ( $\mathrm{n}=263)$; cyclosporin $(\mathrm{n}=266)$

alopecia - 20; 6

anaemia - 47; 38

anorexia - 34; 24

diarrhea $-72 ; 47$

fever $-48 ; 56$

headache - 64; 60

hirsutism - 7; 31

hypertension - 47; 56

nausea $-46 ; 56$ 
paraesthesia - 40; 30

pruritis - 36; 20

rash - 24; 19

tremor - 56; 46

vomiting $27 ; 15$.

\section{Risk of bias}

\begin{tabular}{l|ll}
\hline Item & Authors' judgement & Description \\
\hline Allocation concealment? & Yes & A - Adequate \\
\hline
\end{tabular}

\section{Zervos 1998}

\begin{tabular}{|c|c|}
\hline Methods & $\begin{array}{l}\text { Generation of the allocation sequence: not clear. } \\
\text { Allocation concealment: not clear. } \\
\text { Blinding: not performed. } \\
\text { Follow-up: not clear. Outcomes appear to account for all patients but withdrawals not clearly specified. } \\
\text { Analysis: intention to treat. }\end{array}$ \\
\hline Participants & $\begin{array}{l}\text { Country: USA, single centre. } \\
\text { Language: English. } \\
\text { Inclusion criteria: All male and female patients with hepatitis } C \text { virus undergoing liver transplantation. } \\
\text { Exclusion criteria / diagnoses: not specified. } \\
\text { Allocation: tacrolimus } n=35 \text {; cyclosporin } n=36 \\
\text { - tacrolimus } n=25 \\
\text {-cyclosporin }=24\end{array}$ \\
\hline
\end{tabular}

Interventions
$\begin{aligned} & \text { Tacrolimus protocol: } \\ & -0.05 \mathrm{mg} / \mathrm{kg} \text { po, adjusted to maintain a blood level of } 15 \mathrm{ng} / \mathrm{ml} . \\ & \text { Cyclosporin protocol: } \\ & - \text { cyclosporin formulation: microemulsion } \\ & -3 \mathrm{mg} / \mathrm{kg} \text { po, adjusted to maintain a blood level from } 200 \text { to } 300 \mathrm{ng} / \mathrm{ml} . \\ & \text { Concomitant immunosuppression: steroids all patients. }\end{aligned}$

\begin{tabular}{|l|l}
\hline Outcomes & - patient survival \\
& - graft survival \\
& - acute rejection \\
& - steroid resistant rejection \\
& - withdrawal \\
& - PTLD.
\end{tabular}

Notes

Follow-up: 417 days median (range: from 25 to 625 days).

Other adverse events:

Event - tacrolimus ( $\mathrm{n}=25)$; cyclosporin $(\mathrm{n}=25)$

CMV - 5; 6

Infections - 17; 17

headaches $-2 ; 0$ 
Zervos 1998 (Continued)

Seizures - 0; 4

Aphasia - 0; 1 .

\section{Risk of bias}

\begin{tabular}{l|ll}
\hline Item & Authors' judgement & Description \\
\hline Allocation concealment? & Unclear & B - Unclear \\
\hline
\end{tabular}

Other adverse events: adverse events reported in studies that are not included in the outcomes section of this review are reported in Notes.

CMV: cytomegalovirus

MMF: mycophenolate mofetil

PGE1: prostaglandin E1

plt: platelet count

LOS: length of stay

SBP: spontaneous bacterial peritonitis

od: once daily

po: per os or orally administered medication

q12h: every 12 hours

HIV: human immunodeficiency virus

NG: nasogastric

NJ: nasojejunal

SD: standard deviation

Characteristics of excluded studies [ordered by study ID]

Arnold 1995 Review of published studies.

Ericzon 1997 Specialised data only available at 10 days post-operatively.

Loinaz 2001 Locally derived data merged with local site analysis of data contributed to included multicentred study.

Trull 2002 Follow-up to 1 month postoperatively looking at specialised data. 
DATA AND ANALYSES

Comparison 1. Cyclosporin versus tacrolimus

\begin{tabular}{|c|c|c|c|c|}
\hline Outcome or subgroup title & $\begin{array}{l}\text { No. of } \\
\text { studies }\end{array}$ & $\begin{array}{c}\text { No. of } \\
\text { participants }\end{array}$ & Statistical method & Effect size \\
\hline 1 Mortality & 16 & 3813 & Risk Ratio (M-H, Fixed, 95\% CI) & $0.85[0.73,0.99]$ \\
\hline 2 Graft loss & 16 & 3813 & Risk Ratio (M-H, Fixed, 95\% CI) & $0.78[0.68,0.89]$ \\
\hline 3 Acute rejection & 16 & 3786 & Risk Ratio (M-H, Fixed, 95\% CI) & $0.82[0.77,0.88]$ \\
\hline 4 Steroid-resistent rejection & 11 & 2439 & Risk Ratio (M-H, Fixed, 95\% CI) & $0.57[0.46,0.71]$ \\
\hline $\begin{array}{l}5 \text { Dialysis (de-novo requirement } \\
\text { post-transplantation) }\end{array}$ & 5 & 873 & Risk Ratio (M-H, Fixed, 95\% CI) & $1.55[0.64,3.78]$ \\
\hline $\begin{array}{l}6 \text { Creatinine }(\mathrm{umol} / \mathrm{L}) \text { before } \\
\text { transplantation }\end{array}$ & 2 & 672 & Mean Difference (IV, Fixed, 95\% CI) & $1.36[-3.05,5.77]$ \\
\hline $\begin{array}{l}7 \text { Creatinine (umol/L) } 12 \text { months } \\
\text { after transplantation }\end{array}$ & 2 & 672 & Mean Difference (IV, Fixed, 95\% CI) & $-2.67[-9.55,4.22]$ \\
\hline $\begin{array}{l}8 \text { Diabetes mellitus: initially } \\
\text { diagnosed after transplantation }\end{array}$ & 11 & 3023 & Risk Ratio (M-H, Fixed, 95\% CI) & $1.27[1.12,1.44]$ \\
\hline $\begin{array}{l}9 \text { Post transplant } \\
\text { lymphoproliferative disease }\end{array}$ & 7 & 1107 & Risk Ratio (M-H, Fixed, 95\% CI) & $1.01[0.36,2.86]$ \\
\hline $\begin{array}{l}10 \text { Patients withdrawn from } \\
\text { tacrolimus or cyclosporin }\end{array}$ & 13 & 3156 & Risk Ratio (M-H, Fixed, 95\% CI) & $0.65[0.57,0.74]$ \\
\hline
\end{tabular}

Comparison 2. Stratified analysis, by cyclosporin formulation

\begin{tabular}{|c|c|c|c|c|}
\hline Outcome or subgroup title & $\begin{array}{l}\text { No. of } \\
\text { studies }\end{array}$ & $\begin{array}{c}\text { No. of } \\
\text { participants }\end{array}$ & Statistical method & Effect size \\
\hline 1 Mortality & 16 & 3813 & Risk Ratio (M-H, Fixed, 95\% CI) & $0.85[0.73,0.99]$ \\
\hline $\begin{array}{l}1.1 \text { Tacrolimus versus oil- } \\
\text { based cyclosporin }\end{array}$ & 3 & 1139 & Risk Ratio (M-H, Fixed, 95\% CI) & $0.80[0.61,1.04]$ \\
\hline $\begin{array}{l}\text { 1.2 Tacrolimus versus micro- } \\
\text { emulsion cyclosporin }\end{array}$ & 13 & 2674 & Risk Ratio (M-H, Fixed, 95\% CI) & $0.87[0.73,1.05]$ \\
\hline 2 Graft loss & 16 & 3813 & Risk Ratio (M-H, Fixed, 95\% CI) & $0.78[0.68,0.89]$ \\
\hline $\begin{array}{l}2.1 \text { Tacrolimus versus oil- } \\
\text { based cyclosporin }\end{array}$ & 3 & 1139 & Risk Ratio (M-H, Fixed, 95\% CI) & $0.80[0.64,0.99]$ \\
\hline $\begin{array}{l}2.2 \text { Tacrolimus versus micro- } \\
\text { emulsion cyclosporin }\end{array}$ & 13 & 2674 & Risk Ratio (M-H, Fixed, 95\% CI) & $0.77[0.65,0.92]$ \\
\hline 3 Acute rejection & 16 & 3786 & Risk Ratio (M-H, Fixed, 95\% CI) & $0.82[0.77,0.88]$ \\
\hline $\begin{array}{l}3.1 \text { Tacrolimus versus oil- } \\
\text { based cyclosporin }\end{array}$ & 3 & 1134 & Risk Ratio (M-H, Fixed, 95\% CI) & $0.83[0.75,0.92]$ \\
\hline $\begin{array}{l}3.2 \text { Tacrolimus versus } \\
\text { microemulsion cyclosporin }\end{array}$ & 13 & 2652 & Risk Ratio (M-H, Fixed, 95\% CI) & $0.82[0.75,0.90]$ \\
\hline 4 Steroid-resistent rejection & 11 & 2439 & Risk Ratio (M-H, Fixed, 95\% CI) & $0.57[0.46,0.71]$ \\
\hline $\begin{array}{l}\text { 4.1 Tacrolimus versus oil- } \\
\text { based cyclosporin }\end{array}$ & 3 & 1139 & Risk Ratio (M-H, Fixed, 95\% CI) & $0.49[0.37,0.66$ \\
\hline
\end{tabular}

Cyclosporin versus tacrolimus for liver transplanted patients (Review)

Copyright $(2009$ The Cochrane Collaboration. Published by John Wiley \& Sons, Ltd. 


\begin{tabular}{|c|c|c|c|c|}
\hline $\begin{array}{l}4.2 \text { Tacrolimus versus } \\
\text { microemulsion cyclosporin }\end{array}$ & 8 & 1300 & Risk Ratio (M-H, Fixed, 95\% CI) & $0.67[0.49,0.92]$ \\
\hline $\begin{array}{l}5 \text { Dialysis (de-novo requirement } \\
\text { post-transplantation) }\end{array}$ & 5 & 873 & Risk Ratio (M-H, Fixed, 95\% CI) & $1.55[0.64,3.78]$ \\
\hline $\begin{array}{l}5.1 \text { Tacrolimus versus oil- } \\
\text { based cyclosporin }\end{array}$ & 2 & 610 & Risk Ratio (M-H, Fixed, 95\% CI) & $1.03[0.07,15.83]$ \\
\hline $\begin{array}{l}5.2 \text { Tacrolimus versus } \\
\text { microemulsion cyclosporin }\end{array}$ & 3 & 263 & Risk Ratio (M-H, Fixed, 95\% CI) & $1.63[0.63,4.20]$ \\
\hline $\begin{array}{l}6 \text { Diabetes mellitus: initially } \\
\text { diagnosed after transplantation }\end{array}$ & 11 & 3023 & Risk Ratio (M-H, Fixed, 95\% CI) & $1.27[1.12,1.44]$ \\
\hline $\begin{array}{l}6.1 \text { Tacrolimus versus oil- } \\
\text { based cyclosporin }\end{array}$ & 2 & 1058 & Risk Ratio (M-H, Fixed, 95\% CI) & $1.13[0.99,1.29]$ \\
\hline $\begin{array}{l}6.2 \text { Tacrolimus versus } \\
\text { microemulsion cyclosporin }\end{array}$ & 9 & 1965 & Risk Ratio (M-H, Fixed, 95\% CI) & $1.57[1.20,2.06]$ \\
\hline $\begin{array}{l}7 \text { Post transplant } \\
\text { lymphoproliferative disease }\end{array}$ & 7 & 1107 & Risk Ratio (M-H, Fixed, 95\% CI) & $1.01[0.36,2.86]$ \\
\hline $\begin{array}{l}7.1 \text { Tacrolimus versus oil- } \\
\text { based cyclosporin }\end{array}$ & 2 & 610 & Risk Ratio (M-H, Fixed, 95\% CI) & $0.20[0.02,1.72]$ \\
\hline $\begin{array}{l}7.2 \text { Tacrolimus versus } \\
\text { microemulsion cyclosporin }\end{array}$ & 5 & 497 & Risk Ratio (M-H, Fixed, 95\% CI) & $3.02[0.62,14.71]$ \\
\hline $\begin{array}{l}8 \text { Patients withdrawn from } \\
\text { tacrolimus or cyclosporin }\end{array}$ & 13 & 3156 & Risk Ratio (M-H, Fixed, 95\% CI) & $0.65[0.57,0.74]$ \\
\hline $\begin{array}{l}8.1 \text { Tacrolimus versus oil- } \\
\text { based cyclosporin }\end{array}$ & 3 & 1212 & Risk Ratio (M-H, Fixed, 95\% CI) & $0.75[0.63,0.90]$ \\
\hline $\begin{array}{l}8.2 \text { Tacrolimus versus } \\
\text { microemulsion cyclosporin }\end{array}$ & 10 & 1944 & Risk Ratio (M-H, Fixed, 95\% CI) & $0.53[0.42,0.66]$ \\
\hline
\end{tabular}

Comparison 3. Stratified analysis, by inclusion of children

\begin{tabular}{|c|c|c|c|c|}
\hline Outcome or subgroup title & $\begin{array}{l}\text { No. of } \\
\text { studies }\end{array}$ & $\begin{array}{c}\text { No. of } \\
\text { participants }\end{array}$ & Statistical method & Effect size \\
\hline 1 Mortality & 16 & 3813 & Risk Ratio (M-H, Fixed, 95\% CI) & $0.85[0.73,0.99]$ \\
\hline $\begin{array}{l}1.1 \text { Studies of children or } \\
\text { including children }\end{array}$ & 2 & 714 & Risk Ratio (M-H, Fixed, 95\% CI) & $0.91[0.61,1.38]$ \\
\hline 1.2 Studies excluding children & 14 & 3099 & Risk Ratio (M-H, Fixed, 95\% CI) & $0.84[0.71,0.99]$ \\
\hline 2 Graft loss & 16 & 3813 & Risk Ratio (M-H, Fixed, 95\% CI) & $0.78[0.68,0.89]$ \\
\hline $\begin{array}{l}2.1 \text { Studies of children or } \\
\text { including children }\end{array}$ & 2 & 714 & Risk Ratio (M-H, Fixed, 95\% CI) & $0.82[0.59,1.13]$ \\
\hline 2.2 Studies excluding children & 14 & 3099 & Risk Ratio (M-H, Fixed, 95\% CI) & $0.77[0.67,0.90]$ \\
\hline 3 Acute rejection & 16 & 3786 & Risk Ratio (M-H, Fixed, 95\% CI) & $0.82[0.77,0.88]$ \\
\hline $\begin{array}{l}3.1 \text { Studies of children or } \\
\text { including children }\end{array}$ & 2 & 716 & Risk Ratio (M-H, Fixed, 95\% CI) & $0.87[0.77,0.99]$ \\
\hline 3.2 Studies excluding children & 14 & 3070 & Risk Ratio (M-H, Fixed, 95\% CI) & $0.81[0.74,0.88]$ \\
\hline 4 Steroid-resistent rejection & 11 & 2439 & Risk Ratio (M-H, Fixed, 95\% CI) & $0.57[0.46,0.71]$ \\
\hline $\begin{array}{l}\text { 4.1 Studies of children or } \\
\text { including children }\end{array}$ & 2 & 664 & Risk Ratio (M-H, Fixed, 95\% CI) & $0.52[0.38,0.71]$ \\
\hline 4.2 Studies excluding children & 9 & 1775 & Risk Ratio (M-H, Fixed, 95\% CI) & $0.63[0.47,0.84]$ \\
\hline
\end{tabular}




\begin{tabular}{|c|c|c|c|c|}
\hline $\begin{array}{l}5 \text { Dialysis (de-novo requirement } \\
\text { post-transplantation) }\end{array}$ & 5 & 873 & Risk Ratio (M-H, Fixed, 95\% CI) & $1.55[0.64,3.78]$ \\
\hline $\begin{array}{l}5.1 \text { Studies of children or } \\
\text { including children }\end{array}$ & 1 & 529 & Risk Ratio (M-H, Fixed, 95\% CI) & Not estimable \\
\hline 5.2 Studies excluding children & 4 & 344 & Risk Ratio (M-H, Fixed, 95\% CI) & $1.55[0.64,3.78]$ \\
\hline $\begin{array}{l}6 \text { Diabetes mellitus: initially } \\
\text { diagnosed after transplantation }\end{array}$ & 11 & 3023 & Risk Ratio (M-H, Fixed, 95\% CI) & $1.27[1.12,1.44]$ \\
\hline $\begin{array}{l}\text { 6.1 Studies of children or } \\
\text { including children }\end{array}$ & 2 & 714 & Risk Ratio (M-H, Fixed, 95\% CI) & $1.88[0.76,4.65]$ \\
\hline 6.2 Studies excluding children & 9 & 2309 & Risk Ratio (M-H, Fixed, 95\% CI) & $1.25[1.10,1.42]$ \\
\hline $\begin{array}{l}7 \text { Post transplant } \\
\text { lymphoproliferative disease }\end{array}$ & 7 & 1107 & Risk Ratio (M-H, Fixed, 95\% CI) & $1.01[0.36,2.86]$ \\
\hline $\begin{array}{l}\text { 7.1 Studies of children or } \\
\text { including children }\end{array}$ & 2 & 714 & Risk Ratio (M-H, Fixed, 95\% CI) & $1.59[0.42,6.02]$ \\
\hline 7.2 Studies excluding children & 5 & 393 & Risk Ratio (M-H, Fixed, 95\% CI) & $0.43[0.07,2.89]$ \\
\hline $\begin{array}{l}8 \text { Patients withdrawn from } \\
\text { tacrolimus or cyclosporin }\end{array}$ & 13 & 3156 & Risk Ratio (M-H, Fixed, 95\% CI) & $0.65[0.57,0.74]$ \\
\hline $\begin{array}{l}\text { 8.1 Studies of children or } \\
\text { including children }\end{array}$ & 2 & 710 & Risk Ratio (M-H, Fixed, 95\% CI) & $0.72[0.59,0.89]$ \\
\hline 8.2 Studies excluding children & 11 & 2446 & Risk Ratio (M-H, Fixed, 95\% CI) & $0.61[0.50,0.73]$ \\
\hline
\end{tabular}

Comparison 4. Stratified analysis, by studies reporting 12 month data

\begin{tabular}{|c|c|c|c|c|}
\hline Outcome or subgroup title & $\begin{array}{l}\text { No. of } \\
\text { studies }\end{array}$ & $\begin{array}{c}\text { No. of } \\
\text { participants }\end{array}$ & Statistical method & Effect size \\
\hline 1 Mortality & 16 & 3813 & Risk Ratio (M-H, Fixed, 95\% CI) & $0.85[0.73,0.99]$ \\
\hline $\begin{array}{l}1.1 \text { Studies reporting less than } \\
12 \text { month data }\end{array}$ & 2 & 214 & Risk Ratio (M-H, Fixed, 95\% CI) & $1.22[0.53,2.82]$ \\
\hline $\begin{array}{l}1.2 \text { Studies reporting } 12 \\
\text { month data }\end{array}$ & 14 & 3599 & Risk Ratio (M-H, Fixed, 95\% CI) & $0.84[0.72,0.98]$ \\
\hline 2 Graft loss & 16 & 3813 & Risk Ratio (M-H, Fixed, 95\% CI) & $0.78[0.68,0.89]$ \\
\hline $\begin{array}{l}2.1 \text { Studies reporting less than } \\
12 \text { month data }\end{array}$ & 3 & 709 & Risk Ratio (M-H, Fixed, 95\% CI) & $1.04[0.70,1.53]$ \\
\hline $\begin{array}{l}2.2 \text { Studies reporting } 12 \\
\text { month data }\end{array}$ & 13 & 3104 & Risk Ratio (M-H, Fixed, 95\% CI) & $0.75[0.65,0.87]$ \\
\hline 3 Acute rejection & 16 & 3786 & Risk Ratio (M-H, Fixed, 95\% CI) & $0.82[0.77,0.88]$ \\
\hline $\begin{array}{l}3.1 \text { Studies reporting less than } \\
12 \text { month data }\end{array}$ & 2 & 192 & Risk Ratio (M-H, Fixed, 95\% CI) & $0.83[0.59,1.16]$ \\
\hline $\begin{array}{l}3.2 \text { Studies reporting } 12 \\
\text { month data }\end{array}$ & 14 & 3594 & Risk Ratio (M-H, Fixed, 95\% CI) & $0.82[0.77,0.89]$ \\
\hline 4 Steroid-resistent rejection & 11 & 2573 & Risk Ratio (M-H, Fixed, 95\% CI) & $0.57[0.46,0.70]$ \\
\hline $\begin{array}{l}\text { 4.1 Studies reporting less than } \\
12 \text { month data }\end{array}$ & 1 & 134 & Risk Ratio (M-H, Fixed, 95\% CI) & $0.42[0.14,1.29]$ \\
\hline $\begin{array}{l}\text { 4.2 Studies reporting } 12 \\
\text { month data }\end{array}$ & 11 & 2439 & Risk Ratio (M-H, Fixed, 95\% CI) & $0.57[0.46,0.71]$ \\
\hline $\begin{array}{l}6 \text { Diabetes mellitus: initially } \\
\text { diagnosed after transplantation }\end{array}$ & 11 & 3023 & Risk Ratio (M-H, Fixed, 95\% CI) & $1.27[1.12,1.44]$ \\
\hline
\end{tabular}


6.1 Studies reporting less than

12 month data

6.2 Studies reporting 12

month data

8 Patients withdrawn from

tacrolimus or cyclosporin

8.1 Studies reporting less than

12 month data

8.2 Studies reporting 12

month data

\section{Comparison 5. Stratified analysis, by studies confined to patients with hepatitis $\mathrm{C}$ virus}

\begin{tabular}{|c|c|c|c|c|}
\hline Outcome or subgroup title & $\begin{array}{l}\text { No. of } \\
\text { studies }\end{array}$ & $\begin{array}{c}\text { No. of } \\
\text { participants }\end{array}$ & Statistical method & Effect size \\
\hline 1 Mortality & 16 & 3813 & Risk Ratio (M-H, Fixed, 95\% CI) & $0.85[0.73,0.99]$ \\
\hline $\begin{array}{l}\text { 1.1 Studies confined to } \\
\text { patients with hepatitis } C \text { virus }\end{array}$ & 2 & 128 & Risk Ratio (M-H, Fixed, 95\% CI) & $0.83[0.44,1.56]$ \\
\hline $\begin{array}{l}\text { 1.2 Studies including hepatitis } \\
\mathrm{C} \text { virus and other diagnoses }\end{array}$ & 14 & 3685 & Risk Ratio (M-H, Fixed, 95\% CI) & $0.85[0.73,0.99]$ \\
\hline 2 Graft loss & 16 & 3813 & Risk Ratio (M-H, Fixed, 95\% CI) & $0.78[0.68,0.89]$ \\
\hline $\begin{array}{l}\text { 2.1 Studies confined to } \\
\text { patients with hepatitis } C \text { virus }\end{array}$ & 2 & 128 & Risk Ratio (M-H, Fixed, 95\% CI) & $0.69[0.40,1.19]$ \\
\hline $\begin{array}{l}2.2 \text { Studies including hepatitis } \\
\mathrm{C} \text { virus and other diagnoses }\end{array}$ & 14 & 3685 & Risk Ratio (M-H, Fixed, 95\% CI) & $0.79[0.69,0.90]$ \\
\hline 3 Acute rejection & 16 & 3786 & Risk Ratio (M-H, Fixed, 95\% CI) & $0.82[0.77,0.88]$ \\
\hline $\begin{array}{l}\text { 3.1 Studies confined to } \\
\text { patients with hepatitis } C \text { virus }\end{array}$ & 2 & 128 & Risk Ratio (M-H, Fixed, 95\% CI) & $0.63[0.38,1.02]$ \\
\hline $\begin{array}{l}\text { 3.2 Studies including hepatitis } \\
\mathrm{C} \text { virus and other diagnoses }\end{array}$ & 14 & 3658 & Risk Ratio (M-H, Fixed, 95\% CI) & $0.83[0.77,0.89]$ \\
\hline 4 Steroid-resistent rejection & 11 & 2439 & Risk Ratio (M-H, Fixed, 95\% CI) & $0.57[0.46,0.71]$ \\
\hline $\begin{array}{l}\text { 4.1 Studies confined to } \\
\text { patients with hepatitis } C \text { virus }\end{array}$ & 1 & 49 & Risk Ratio (M-H, Fixed, 95\% CI) & $0.32[0.01,7.50]$ \\
\hline $\begin{array}{l}\text { 4.2 Studies including hepatitis } \\
\text { C virus and other diagnoses }\end{array}$ & 10 & 2390 & Risk Ratio (M-H, Fixed, 95\% CI) & $0.58[0.47,0.71]$ \\
\hline $\begin{array}{l}5 \text { Post transplant } \\
\text { lymphoproliferative disease }\end{array}$ & 7 & 1107 & Risk Ratio (M-H, Fixed, 95\% CI) & $1.01[0.36,2.86]$ \\
\hline $\begin{array}{l}5.1 \text { Studies confined to } \\
\text { patients with hepatitis } C \text { virus }\end{array}$ & 1 & 49 & Risk Ratio (M-H, Fixed, 95\% CI) & Not estimable \\
\hline $\begin{array}{l}5.2 \text { Studies including hepatitis } \\
\text { C virus and other diagnoses }\end{array}$ & 6 & 1058 & Risk Ratio (M-H, Fixed, 95\% CI) & $1.01[0.36,2.86]$ \\
\hline $\begin{array}{l}6 \text { Patients withdrawn from } \\
\text { tacrolimus or cyclosporin }\end{array}$ & 13 & 3156 & Risk Ratio (M-H, Fixed, 95\% CI) & $0.65[0.57,0.74]$ \\
\hline $\begin{array}{l}\text { 6.1 Studies confined to } \\
\text { patients with hepatitis } C \text { virus }\end{array}$ & 2 & 128 & Risk Ratio (M-H, Fixed, 95\% CI) & $0.43[0.18,1.02]$ \\
\hline $\begin{array}{l}\text { 6.2 Studies including hepatitis } \\
\text { C virus and other diagnoses }\end{array}$ & 11 & 3028 & Risk Ratio (M-H, Fixed, 95\% CI) & $0.66[0.57,0.76]$ \\
\hline
\end{tabular}




\begin{tabular}{|c|c|c|c|c|}
\hline Outcome or subgroup title & $\begin{array}{l}\text { No. of } \\
\text { studies }\end{array}$ & $\begin{array}{c}\text { No. of } \\
\text { participants }\end{array}$ & Statistical method & Effect size \\
\hline 1 Mortality & 16 & 3813 & Risk Ratio (M-H, Fixed, 95\% CI) & $0.85[0.73,0.99]$ \\
\hline $\begin{array}{l}1.1 \text { Concomittant } \\
\text { azathioprine }\end{array}$ & 4 & 868 & Risk Ratio (M-H, Fixed, 95\% CI) & $0.69[0.51,0.92]$ \\
\hline $\begin{array}{l}\text { 1.2 Concomitant azathioprine } \\
\text { (some centres only) }\end{array}$ & 1 & 495 & Risk Ratio (M-H, Fixed, 95\% CI) & $0.94[0.61,1.44]$ \\
\hline $\begin{array}{l}1.3 \text { Concomitant azathioprine } \\
\text { with cyclosporin only (some } \\
\text { centres) }\end{array}$ & 5 & 2006 & Risk Ratio (M-H, Fixed, 95\% CI) & $0.94[0.76,1.16]$ \\
\hline $\begin{array}{l}1.4 \text { Concomitant } \\
\text { mycophenolate mofetil }\end{array}$ & 3 & 250 & Risk Ratio (M-H, Fixed, 95\% CI) & $1.02[0.37,2.80]$ \\
\hline $\begin{array}{l}1.5 \text { No concomitant } \\
\text { azathioprine or mycopholic } \\
\text { mofetil }\end{array}$ & 3 & 194 & Risk Ratio (M-H, Fixed, 95\% CI) & $0.69[0.39,1.24]$ \\
\hline 2 Graft loss & 16 & 3813 & Risk Ratio (M-H, Fixed, 95\% CI) & $0.78[0.68,0.89]$ \\
\hline $\begin{array}{l}2.1 \text { Studies with concomittant } \\
\text { azathioprine }\end{array}$ & 4 & 868 & Risk Ratio (M-H, Fixed, 95\% CI) & $0.65[0.49,0.84]$ \\
\hline $\begin{array}{l}2.2 \text { Concomitant azathioprine } \\
\text { (some centres only) }\end{array}$ & 1 & 495 & Risk Ratio (M-H, Fixed, 95\% CI) & $1.10[0.67,1.80]$ \\
\hline $\begin{array}{l}2.3 \text { Concomitant azathioprine } \\
\text { with cyclosporine only (some } \\
\text { centres) }\end{array}$ & 5 & 2006 & Risk Ratio (M-H, Fixed, 95\% CI) & $0.86[0.72,1.03]$ \\
\hline $\begin{array}{l}2.4 \text { Concomitant } \\
\text { mycophenolate mofetil }\end{array}$ & 3 & 250 & Risk Ratio (M-H, Fixed, 95\% CI) & $0.69[0.30,1.60]$ \\
\hline $\begin{array}{l}2.5 \text { No concomitant } \\
\text { azathioprine or mycophenolate } \\
\text { mofetil }\end{array}$ & 3 & 194 & Risk Ratio (M-H, Fixed, 95\% CI) & $0.55[0.35,0.88]$ \\
\hline 3 Acute rejection & 16 & 3786 & Risk Ratio (M-H, Fixed, 95\% CI) & $0.82[0.77,0.88]$ \\
\hline 3.1 Concomitant azathioprine & 4 & 868 & Risk Ratio (M-H, Fixed, 95\% CI) & $0.83[0.72,0.95]$ \\
\hline $\begin{array}{l}\text { 3.2 Concomitant azathioprine } \\
\text { (some centres only) }\end{array}$ & 1 & 495 & Risk Ratio (M-H, Fixed, 95\% CI) & $0.68[0.33,1.38]$ \\
\hline $\begin{array}{l}3.3 \text { Concomitant azathioprine } \\
\text { with cyclosporin only (some } \\
\text { centres) }\end{array}$ & 5 & 1999 & Risk Ratio (M-H, Fixed, 95\% CI) & $0.85[0.78,0.93]$ \\
\hline $\begin{array}{l}3.4 \text { Concomitant } \\
\text { mycophenolate mofetil }\end{array}$ & 3 & 237 & Risk Ratio (M-H, Fixed, 95\% CI) & $0.77[0.56,1.06]$ \\
\hline $\begin{array}{l}3.5 \text { No concomitant } \\
\text { azathioprine or mycophenolate }\end{array}$ & 3 & 187 & Risk Ratio (M-H, Fixed, 95\% CI) & $0.68[0.53,0.86]$ \\
\hline 4 Steroid-resistent rejection & 11 & 2439 & Risk Ratio (M-H, Fixed, 95\% CI) & $0.57[0.46,0.71]$ \\
\hline 4.1 Concomitant azathioprine & 2 & 183 & Risk Ratio (M-H, Fixed, 95\% CI) & $0.43[0.15,1.26]$ \\
\hline $\begin{array}{l}\text { 4.2 Concomitant azathioprine } \\
\text { with cyclosporin only (some } \\
\text { centres only) }\end{array}$ & 5 & 1947 & Risk Ratio (M-H, Fixed, 95\% CI) & $0.58[0.46,0.74]$ \\
\hline $\begin{array}{l}4.3 \text { Concomitant } \\
\text { mycophenolate mofetil }\end{array}$ & 2 & 179 & Risk Ratio (M-H, Fixed, 95\% CI) & $0.53[0.20,1.37]$ \\
\hline
\end{tabular}


4.4 No concomitant

azathioprine or mycophenolate mofetil

5 Dialysis (de-novo requirement post-transplantation)

5.1 Concomitant azathioprine

5.2 Concomitant azathioprine with cyclosporin only (some

centres only)

5.3 Concomitant mycophenolate mofetil

5.4 No concomitant azathioprine or mycophenolate mofetil

6 Diabetes mellitus: initially diagnosed after transplantation 6.1 Concomitant azathioprine 6.2 Concomitant azathioprine with cyclosporin only (some centres only)

6.3 Concomitant mycophenolate mofetil

7 Post transplant lymphoproliferative disease 7.1 Concomitant azathioprine 7.2 Concomitant azathioprine with cyclosporin only (some centres only)

7.3 Concomitant mycophenolate mofetil

7.4 No concomitant azathioprine or mycophenolate mofetil

8 Patients withdrawn from tacrolimus or cyclosporin 8.1 Concomitant azathioprine 8.2 Concomitant azathioprine with cyclosporin only (some centres only)

8.3 Concomitant mycophenolate mofetil

8.4 No concomitant azathioprine or mycophenolate mofetil
Not estimable $1.59[0.42,6.02]$ Risk Ratio (M-H, Fixed, 95\% CI) 


\section{Analysis I.I. Comparison I Cyclosporin versus tacrolimus, Outcome I Mortality.}

Review: Cyclosporin versus tacrolimus for liver transplanted patients

Comparison: I Cyclosporin versus tacrolimus

Outcome: I Mortality

\begin{tabular}{|c|c|c|c|c|c|}
\hline \multirow[t]{2}{*}{ Study or subgroup } & tacrolimus & cyclosporin & Risk Ratio & \multirow[t]{2}{*}{ Weight } & \multirow{2}{*}{$\begin{array}{r}\text { Risk Ratio } \\
\text { M-H,Fixed,95\% Cl }\end{array}$} \\
\hline & $\mathrm{n} / \mathrm{N}$ & $\mathrm{n} / \mathrm{N}$ & M-H,Fixed,95\% Cl & & \\
\hline European Study 1994 & $46 / 264$ & $61 / 265$ & \pm & $20.3 \%$ & $0.76[0.54,1.07]$ \\
\hline Fisher 1998 & $1 / 49$ & $2 / 50$ & & $0.7 \%$ & $0.51[0.05,5.45]$ \\
\hline Fung 1991 & $3 / 41$ & $7 / 40$ & - & $2.4 \%$ & $0.42[0.12,1.50]$ \\
\hline Grazi 2004 & $34 / 245$ & $37 / 250$ & $\rightarrow$ & $12.2 \%$ & $0.94[0.61,1.44]$ \\
\hline Greig 2003 & $2 / 71$ & $8 / 72$ & 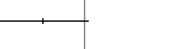 & $2.6 \%$ & $0.25[0.06,1.15]$ \\
\hline Kelly 2004 & $7 / 92$ & 9/93 & 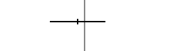 & $3.0 \%$ & $0.79[0.31,2.02]$ \\
\hline Klupp 1999 & $2 / 40$ & $3 / 40$ & - & $1.0 \%$ & $0.67[0.12,3.78]$ \\
\hline Martin 2004 & $6 / 38$ & $8 / 41$ & + & $2.6 \%$ & $0.81[0.31,2.12]$ \\
\hline Muehlbacher 2001 & $47 / 313$ & $37 / 307$ & - & $12.4 \%$ & $1.25[0.83,1.86]$ \\
\hline O'Grady 2002 & $50 / 301$ & $72 / 305$ & 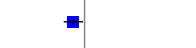 & $23.8 \%$ & $0.70[0.51,0.97]$ \\
\hline Rolles 1999 & $5 / 30$ & $7 / 34$ & 1 & $2.2 \%$ & $0.81[0.29,2.28]$ \\
\hline Stegall 1997 & $4 / 35$ & $2 / 36$ & 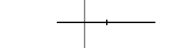 & $0.7 \%$ & $2.06[0.40,10.52]$ \\
\hline Therapondos 2002 & $2 / 20$ & $1 / 20$ & - & $0.3 \%$ & $2.00[0.20,20.33]$ \\
\hline Timmermann 2002 & $7 / 72$ & $7 / 71$ & \begin{tabular}{l|l} 
\\
\end{tabular} & $2.3 \%$ & $0.99[0.36,2.67]$ \\
\hline U. S. Study 1994 & $31 / 263$ & $33 / 266$ & $\rightarrow$ & $10.9 \%$ & $0.95[0.60,1.50]$ \\
\hline Zervos 1998 & $7 / 25$ & $8 / 24$ & $\longrightarrow$ & $2.7 \%$ & $0.84[0.36,1.96]$ \\
\hline Total (95\% CI) & 1899 & 1914 & - & $100.0 \%$ & $0.85[0.73,0.99]$ \\
\hline \multicolumn{6}{|c|}{ Total events: 254 (tacrolimus), 302 (cyclosporin) } \\
\hline \multicolumn{6}{|c|}{ Heterogeneity: $\mathrm{Chi}^{2}=11.33, \mathrm{df}=15(\mathrm{P}=0.73) ; 1^{2}=0.0 \%$} \\
\hline \multicolumn{6}{|c|}{ Test for overall effect: $Z=2.11(P=0.035)$} \\
\hline
\end{tabular}

$\begin{array}{lllll}0.01 & 0.1 & 1 & 10 & 100\end{array}$

Favours tacrolimus Favours cyclosporin 


\section{Analysis I.2. Comparison I Cyclosporin versus tacrolimus, Outcome 2 Graft loss.}

Review: Cyclosporin versus tacrolimus for liver transplanted patients

Comparison: I Cyclosporin versus tacrolimus

Outcome: 2 Graft loss

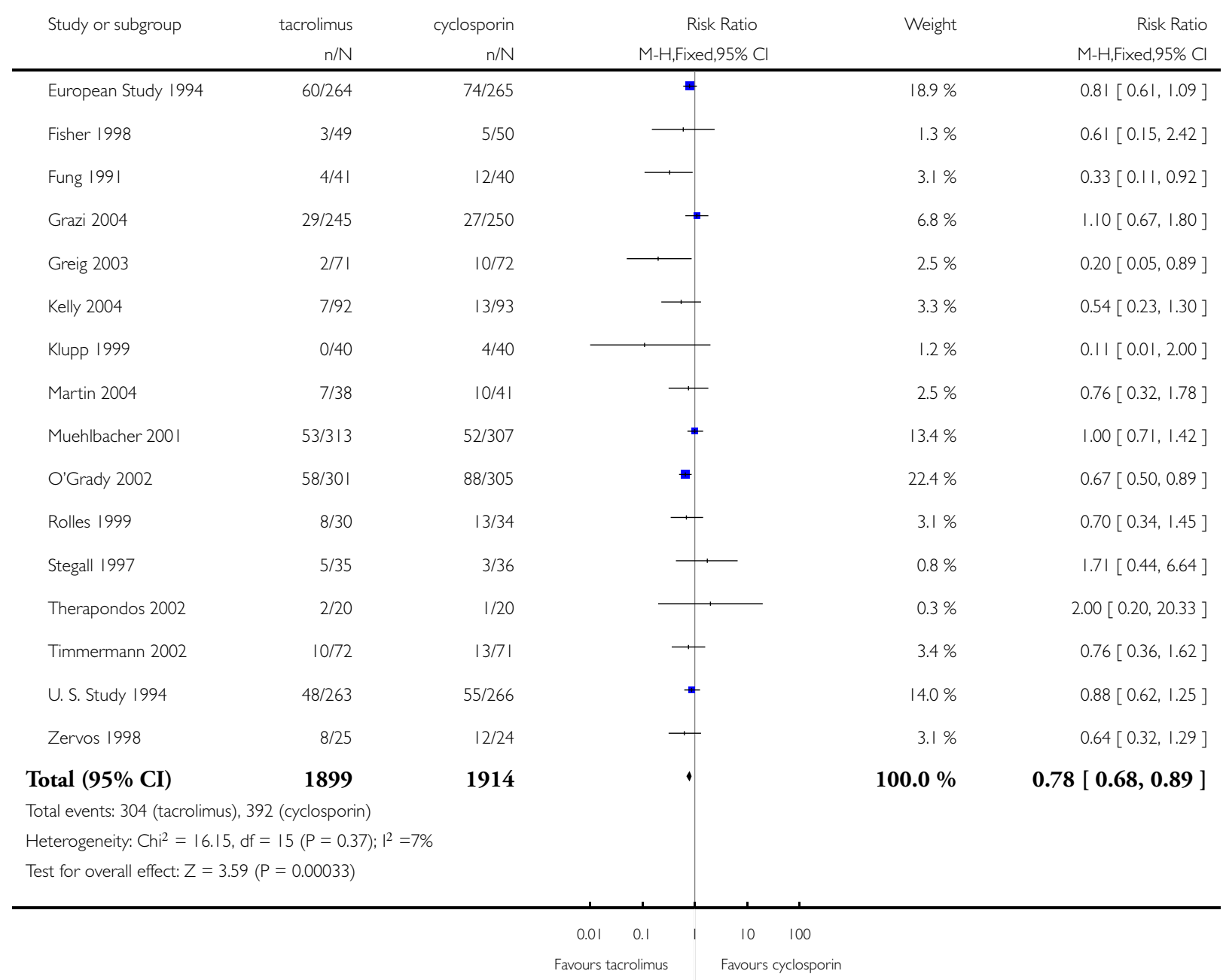




\section{Analysis I.3. Comparison I Cyclosporin versus tacrolimus, Outcome 3 Acute rejection.}

Review: Cyclosporin versus tacrolimus for liver transplanted patients

Comparison: I Cyclosporin versus tacrolimus

Outcome: 3 Acute rejection

\begin{tabular}{|c|c|c|c|c|c|}
\hline \multirow[t]{2}{*}{ Study or subgroup } & tacrolimus & cyclosporin & \multirow{2}{*}{$\begin{array}{c}\text { Risk Ratio } \\
\text { M-H,Fixed,95\% Cl }\end{array}$} & \multirow[t]{2}{*}{ Weight } & \multirow{2}{*}{$\begin{array}{r}\text { Risk Ratio } \\
\text { M-H,Fixed,95\% Cl }\end{array}$} \\
\hline & $\mathrm{n} / \mathrm{N}$ & $\mathrm{n} / \mathrm{N}$ & & & \\
\hline European Study 1994 & $115 / 264$ & $142 / 265$ & \pm & $16.0 \%$ & $0.81[0.68,0.97]$ \\
\hline Fisher 1998 & $5 / 49$ & $2 / 50$ & & $0.2 \%$ & $2.55[0.52,12.53]$ \\
\hline Fung 1991 & $19 / 4 \mid$ & $29 / 33$ & $\longrightarrow$ & $3.6 \%$ & $0.53[0.37,0.75]$ \\
\hline Grazi 2004 & $12 / 245$ & $18 / 250$ & 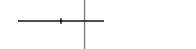 & $2.0 \%$ & $0.68[0.33,1.38]$ \\
\hline Greig 2003 & $25 / 71$ & $31 / 72$ & $\longrightarrow$ & $3.5 \%$ & $0.82[0.54,1.24]$ \\
\hline Kelly 2004 & $38 / 92$ & $49 / 93$ & 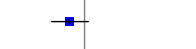 & $5.5 \%$ & $0.78[0.57,1.07]$ \\
\hline Klupp 1999 & $18 / 40$ & $30 / 40$ & $\longrightarrow$ & $3.4 \%$ & $0.60[0.41,0.88]$ \\
\hline Martin 2004 & $11 / 38$ & $|6 / 4|$ & +1 & $1.7 \%$ & $0.74[0.40,1.39]$ \\
\hline Muehlbacher 2001 & $119 / 313$ & $135 / 307$ & - & $15.4 \%$ & $0.86[0.72,1.05]$ \\
\hline O'Grady 2002 & $|43 / 30|$ & $179 / 305$ & $\div$ & $20.1 \%$ & $0.81[0.70,0.94]$ \\
\hline Rolles 1999 & $20 / 30$ & $22 / 34$ & 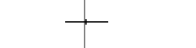 & $2.3 \%$ & $1.03[0.72,1.47]$ \\
\hline Stegall 1997 & $11 / 26$ & $15 / 32$ & + & $1.5 \%$ & $0.90[0.50,1.61]$ \\
\hline Therapondos 2002 & $8 / 20$ & $3 / 20$ & t. & $0.3 \%$ & $2.67[0.82,8.62]$ \\
\hline Timmermann 2002 & $24 / 65$ & $32 / 69$ & + & $3.5 \%$ & $0.80[0.53,1.20]$ \\
\hline U. S. Study 1994 & $154 / 265$ & $173 / 266$ & $=$ & $19.5 \%$ & $0.89[0.78,1.02]$ \\
\hline Zervos 1998 & $6 / 25$ & $12 / 24$ & + & $1.4 \%$ & $0.48[0.21,1.07]$ \\
\hline Total (95\% CI) & 1885 & 1901 & - & $100.0 \%$ & $0.82[0.77,0.88]$ \\
\hline \multicolumn{6}{|c|}{ Total events: 728 (tacrolimus), 888 (cyclosporin) } \\
\hline \multicolumn{6}{|c|}{ Heterogeneity: $\mathrm{Chi}^{2}=20.10, \mathrm{df}=15(P=0.17) ; 1^{2}=25 \%$} \\
\hline \multicolumn{6}{|c|}{ Test for overall effect: $Z=5.43(P<0.0000 \mathrm{I})$} \\
\hline
\end{tabular}

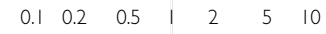

Favours tacrolimus Favours cyclosporin 


\section{Analysis I.4. Comparison I Cyclosporin versus tacrolimus, Outcome 4 Steroid-resistent rejection.}

Review: Cyclosporin versus tacrolimus for liver transplanted patients

Comparison: I Cyclosporin versus tacrolimus

Outcome: 4 Steroid-resistent rejection

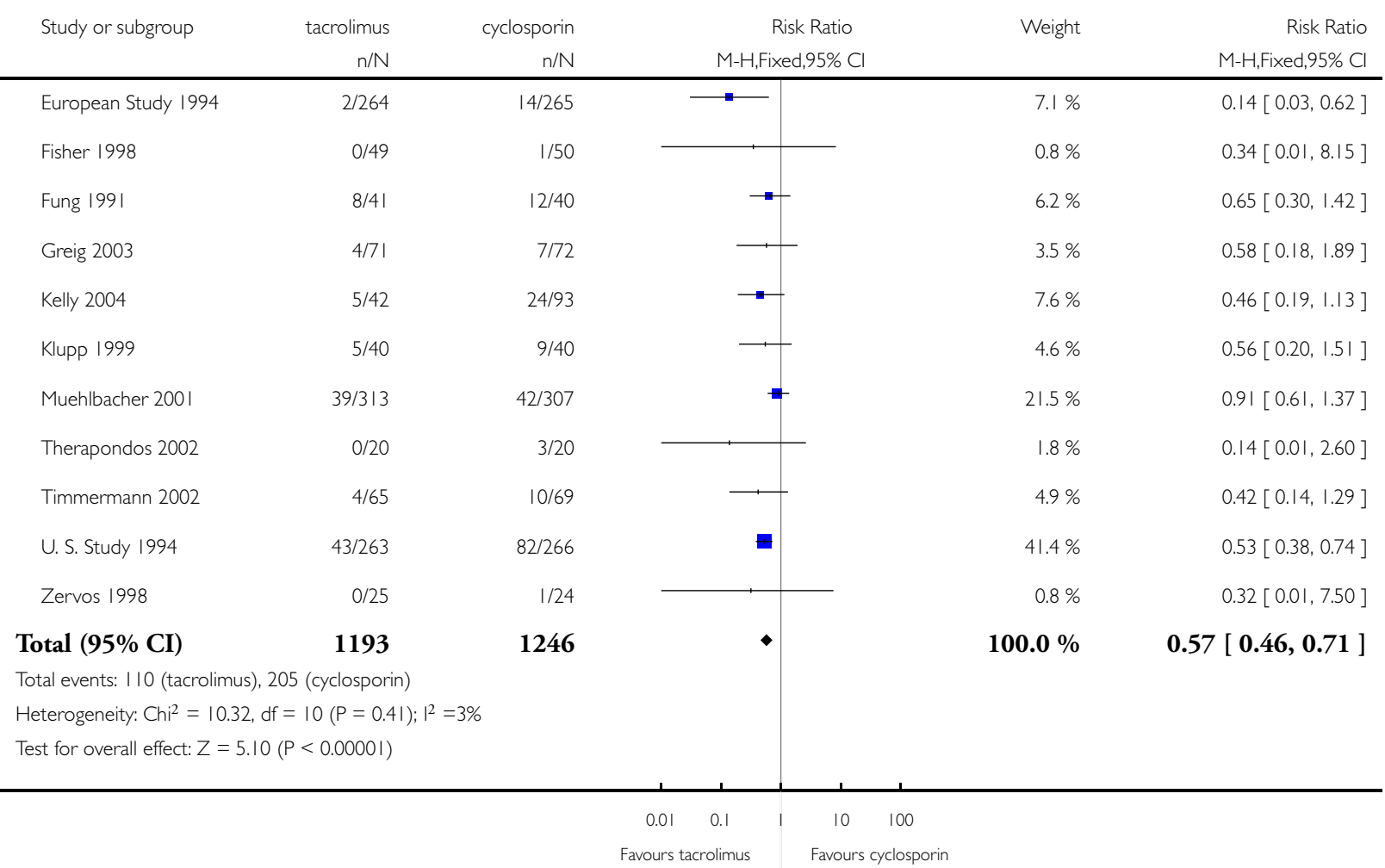


Analysis I.5. Comparison I Cyclosporin versus tacrolimus, Outcome 5 Dialysis (de-novo requirement posttransplantation).

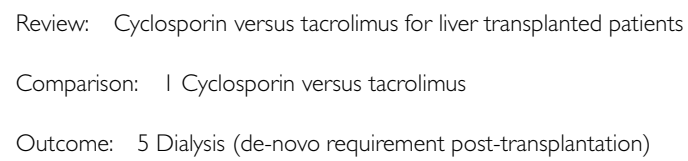

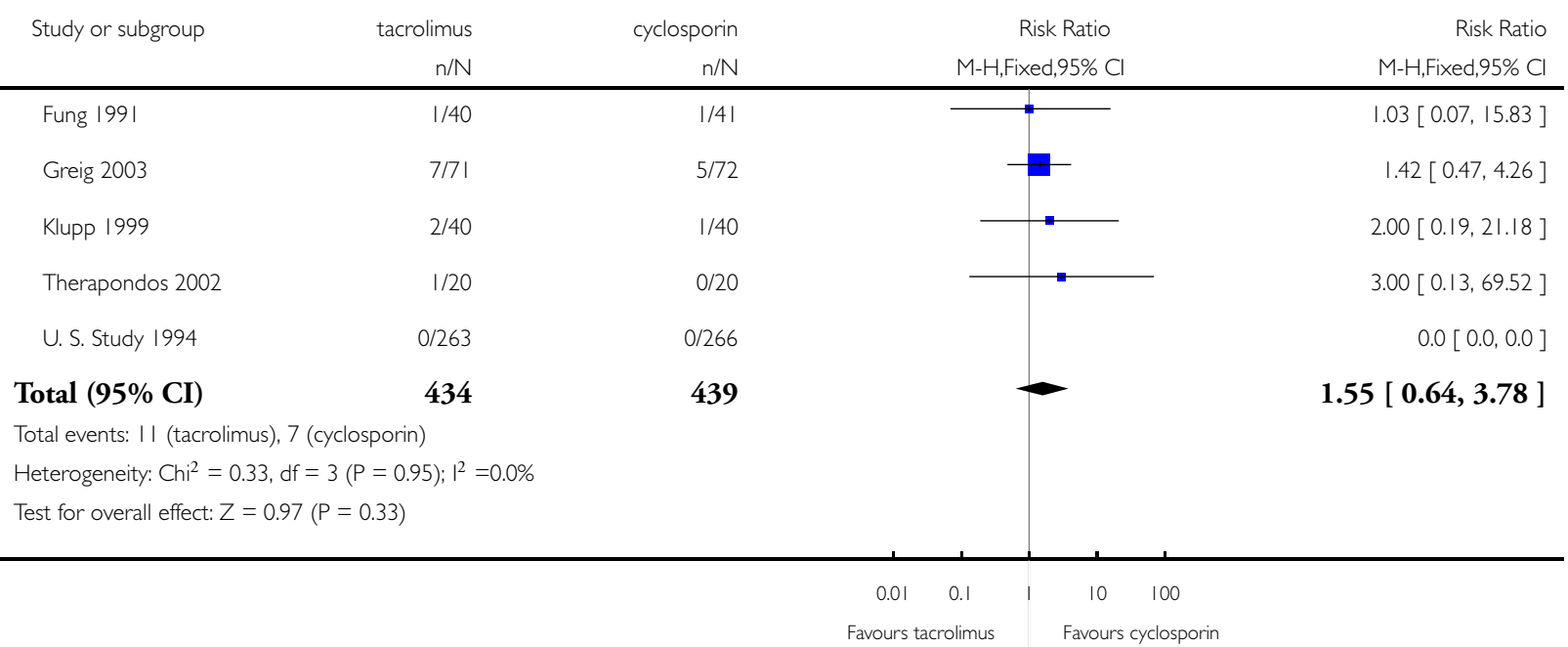

\section{Analysis I.6. Comparison I Cyclosporin versus tacrolimus, Outcome 6 Creatinine (umol/L) before} transplantation.

Review: Cyclosporin versus tacrolimus for liver transplanted patients

Comparison: I Cyclosporin versus tacrolimus

Outcome: 6 Creatinine (umol/L) before transplantation

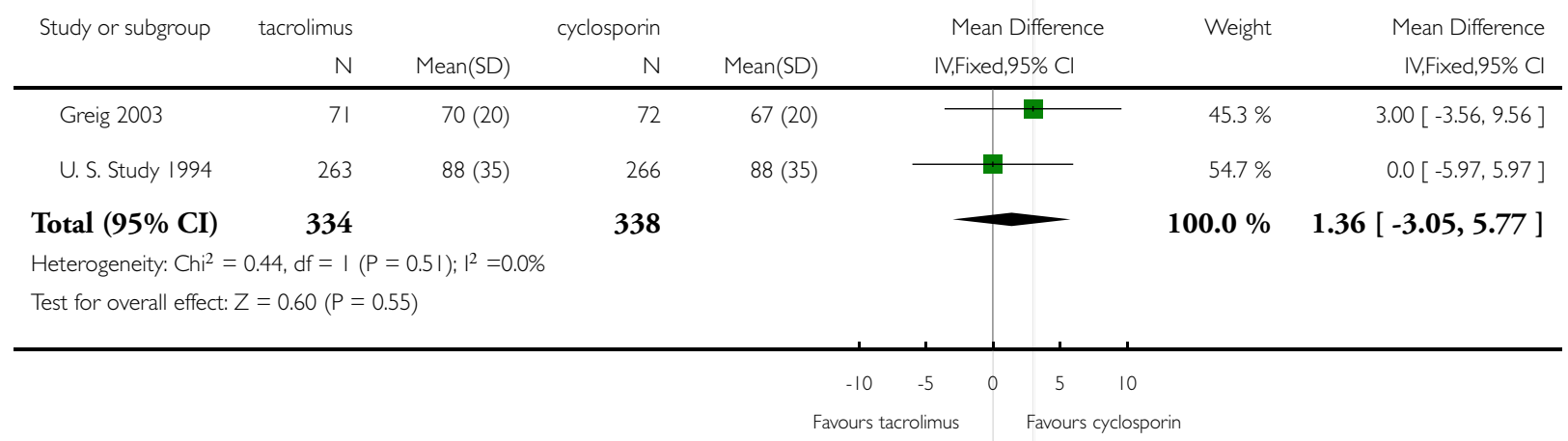




\section{Analysis I.7. Comparison I Cyclosporin versus tacrolimus, Outcome 7 Creatinine (umol/L) I 2 months after transplantation.}

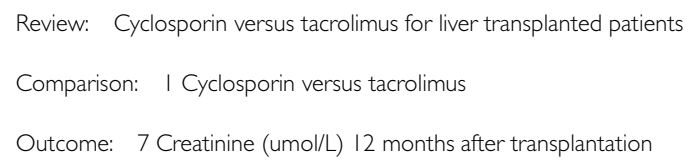

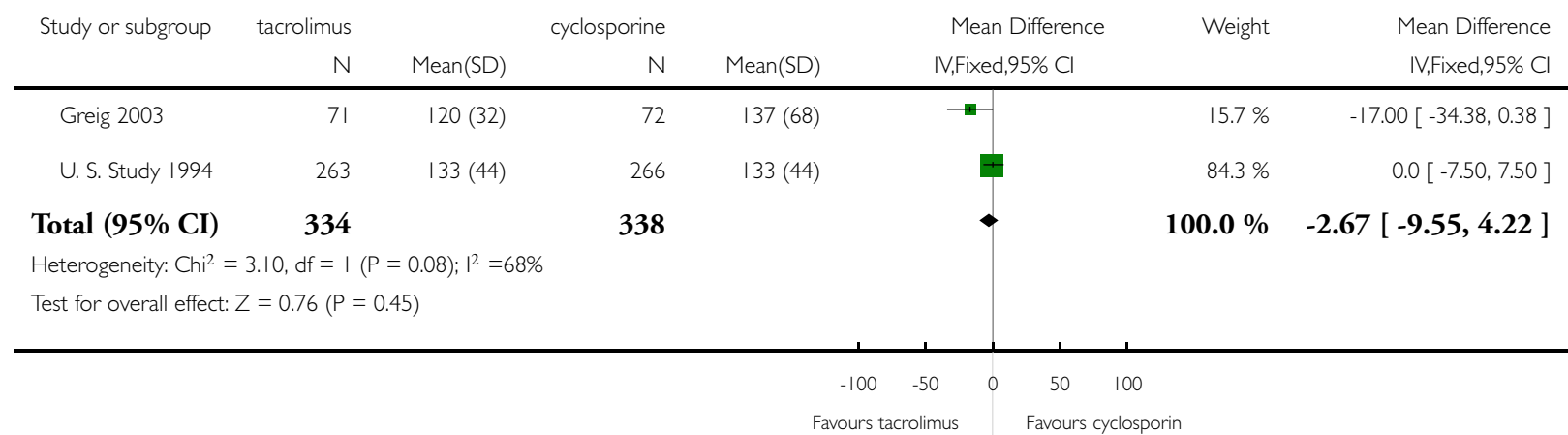

Analysis I.8. Comparison I Cyclosporin versus tacrolimus, Outcome 8 Diabetes mellitus: initially diagnosed after transplantation.

Review: Cyclosporin versus tacrolimus for liver transplanted patients

Comparison: I Cyclosporin versus tacrolimus

Outcome: 8 Diabetes mellitus: initially diagnosed after transplantation

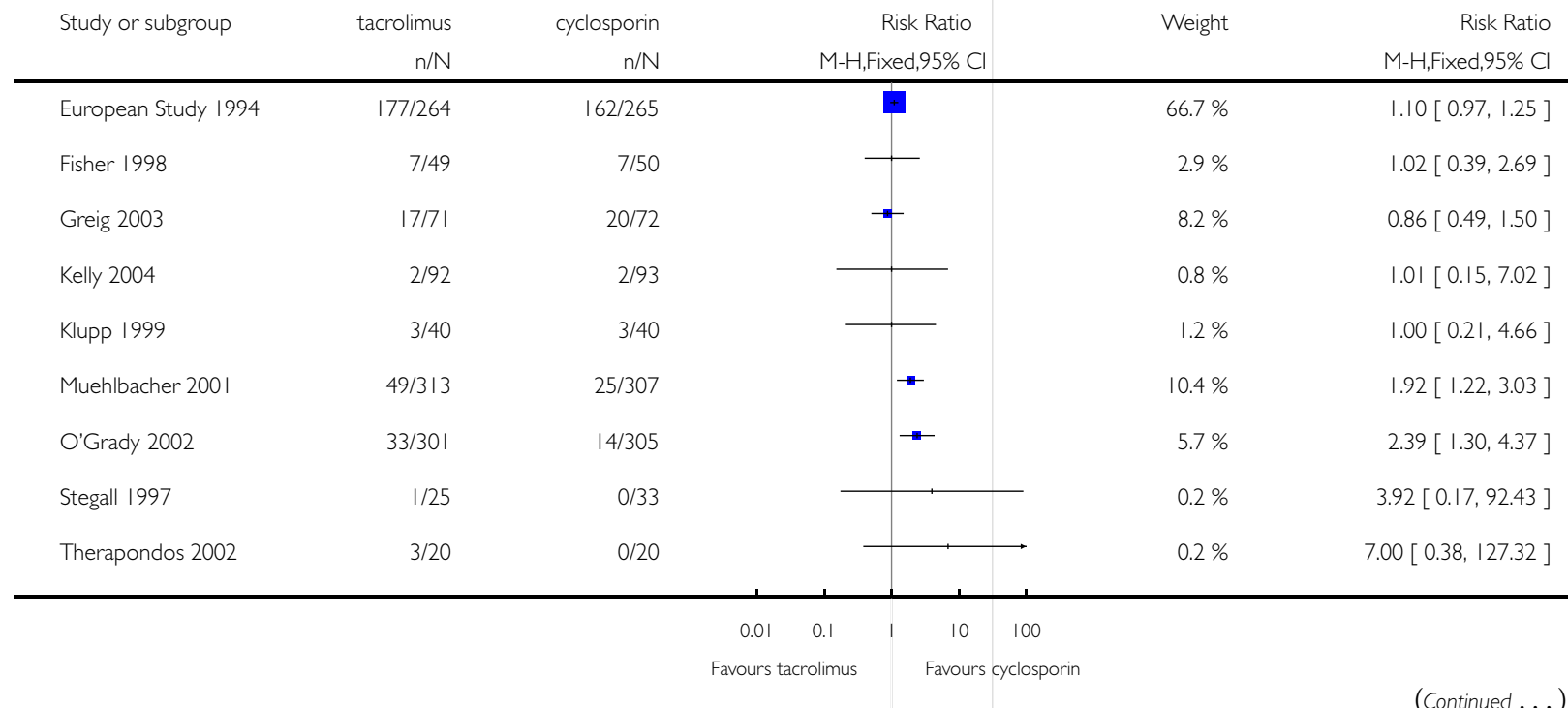




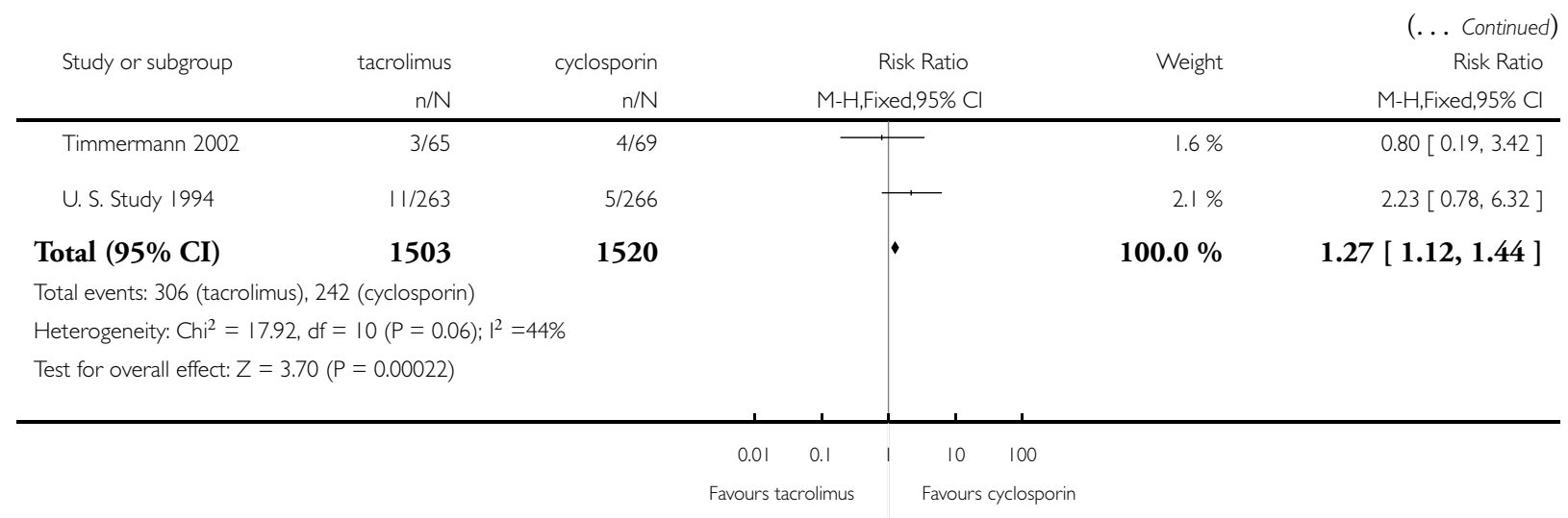

\section{Analysis I.9. Comparison I Cyclosporin versus tacrolimus, Outcome 9 Post transplant lymphoproliferative} disease.

Review: Cyclosporin versus tacrolimus for liver transplanted patients

Comparison: I Cyclosporin versus tacrolimus

Outcome: 9 Post transplant lymphoproliferative disease

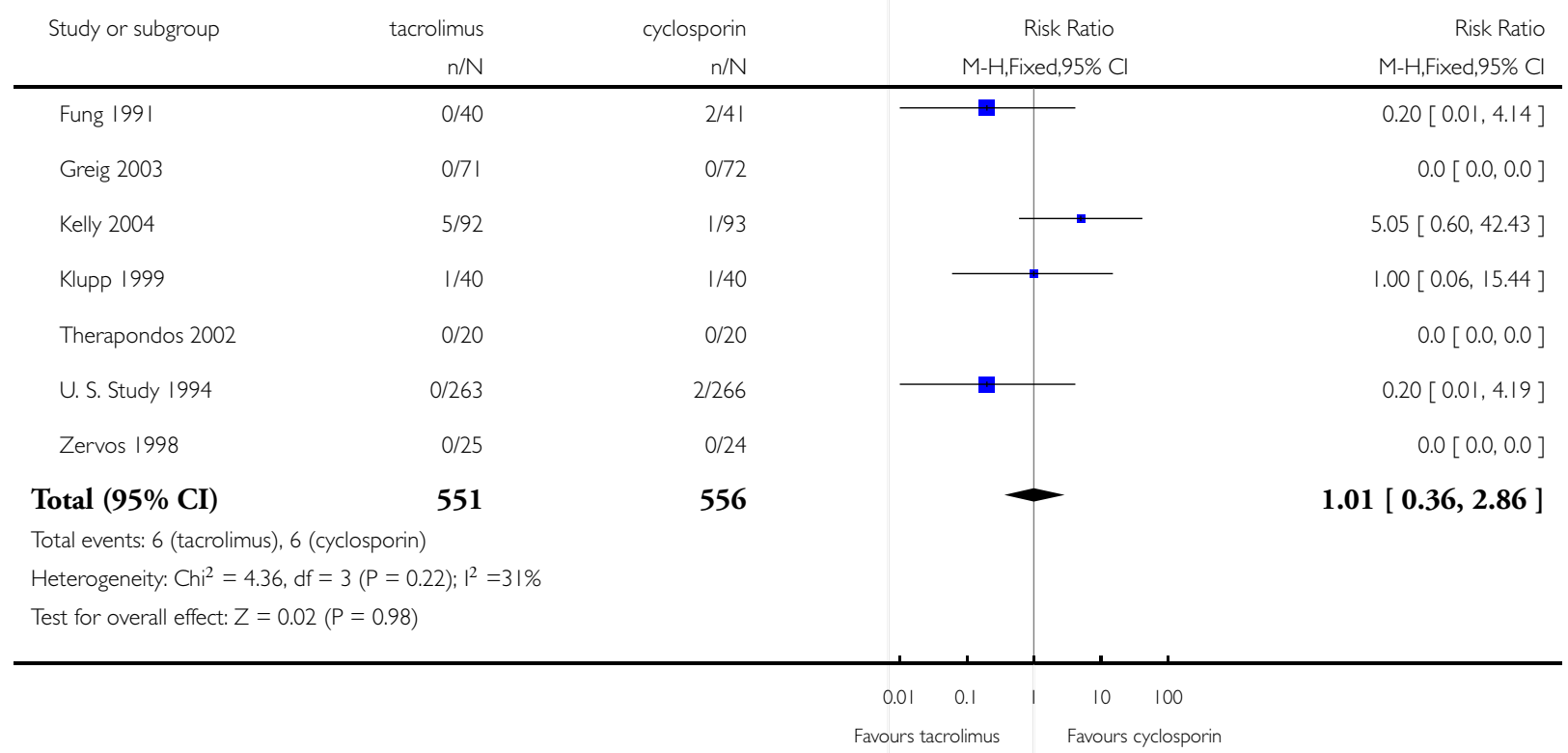




\section{Analysis I.10. Comparison I Cyclosporin versus tacrolimus, Outcome 10 Patients withdrawn from tacrolimus or cyclosporin.}

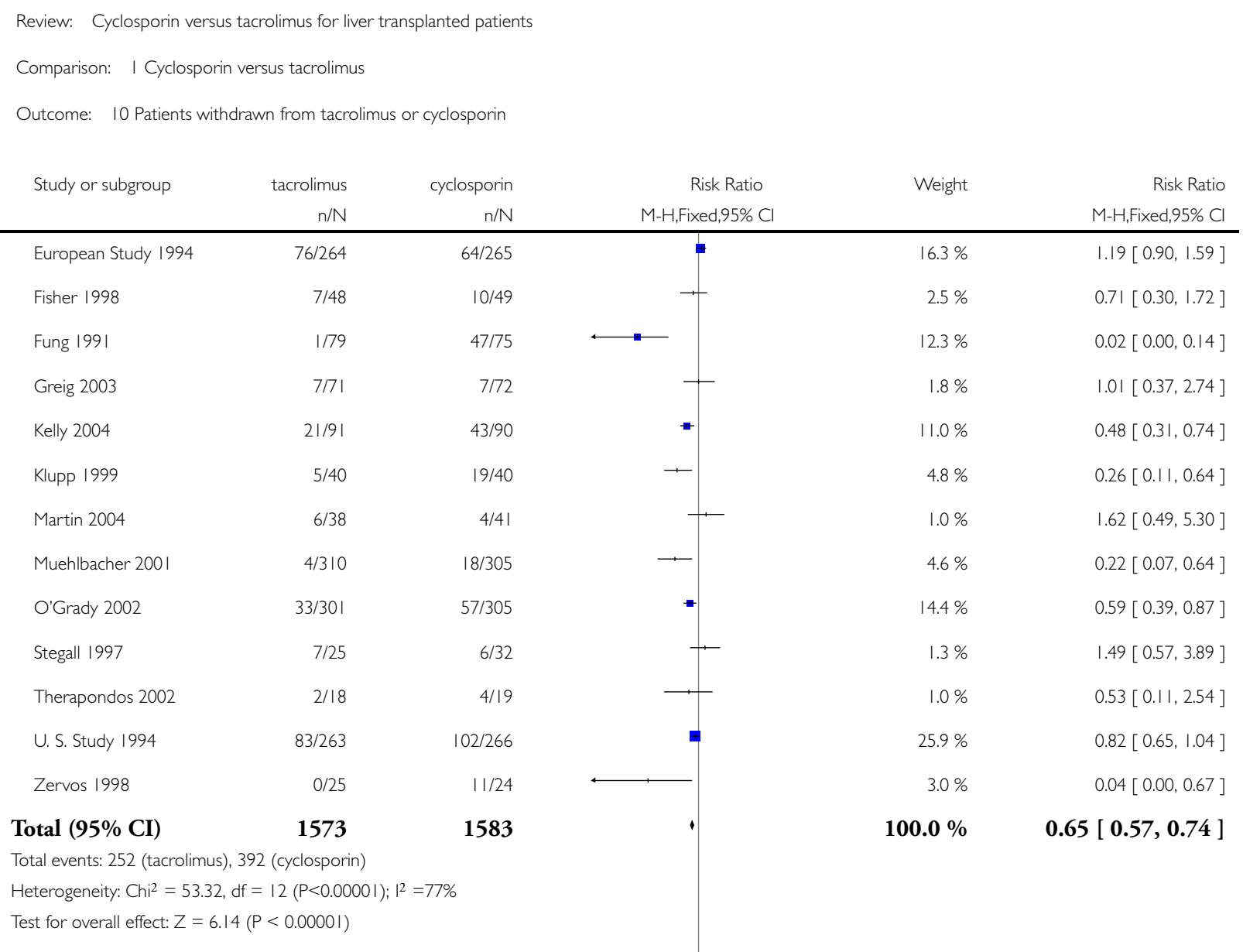

$\begin{array}{lllllll}0.001 & 0.01 & 0.1 \quad 1 & 10 & 100 & 1000\end{array}$

Favours tacrolimus Favours cyclosporin 
Analysis 2.I. Comparison 2 Stratified analysis, by cyclosporin formulation, Outcome I Mortality.

Review: Cyclosporin versus tacrolimus for liver transplanted patients

Comparison: 2 Stratified analysis, by cyclosporin formulation

Outcome: | Mortality

tacrolimus

cyclosporin

Risk Ratio

Weight

Risk Ratio

$\mathrm{n} / \mathrm{N}$

$\mathrm{n} / \mathrm{N}$

M-H,Fixed,95\% Cl

M-H,Fixed,95\% Cl

I Tacrolimus versus oil-based cyclosporin

European Study $1994 \quad$ 46/264

$61 / 265$

Fung 1991

$3 / 41$

$7 / 40$

U. S. Study 1994

$31 / 263$

$33 / 266$

Subtotal (95\% CI)

568

571

Total events: 80 (tacrolimus), 101 (cyclosporin)

Heterogeneity: $\mathrm{Chi}^{2}=1.62, \mathrm{df}=2(\mathrm{P}=0.44) ; \mathrm{I}^{2}=0.0 \%$

Test for overall effect: $Z=1.67(P=0.095)$

2 Tacrolimus versus micro-emulsion cyclosporin

Fisher 1998

1/49 2/50

Grazi 2004

$34 / 245$

$37 / 250$

Greig 2003

$2 / 71$

$8 / 72$

Kelly 2004

$7 / 92$

9/93

Klupp 1999

$2 / 40$

$3 / 40$

Martin 2004

$6 / 38$

$8 / 41$

Muehlbacher 200

$47 / 313$

$37 / 307$

O'Grady 2002

$50 / 301$

$72 / 305$

Rolles 1999

$5 / 30$

$7 / 34$

Stegall 1997

$4 / 35$

2/36

Therapondos 2002

$2 / 20$

I/20

Timmermann 2002

$7 / 72$

$7 / 71$

Zervos 1998

$7 / 25$

$8 / 24$

Subtotal (95\% CI)

1331

1343

Total events: 174 (tacrolimus), 201 (cyclosporin)

Heterogeneity: $\mathrm{Ch}^{2}=9.39, \mathrm{df}=12(\mathrm{P}=0.67) ; \mathrm{I}^{2}=0.0 \%$

Test for overall effect: $Z=1.41(P=0.16)$

Total (95\% CI)

1899

1914

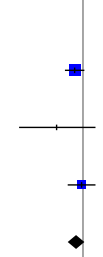

$20.3 \%$

$2.4 \%$

$10.9 \%$

$33.5 \%$

$0.7 \%$

$12.2 \%$

$2.6 \%$

$3.0 \%$

$1.0 \%$

$2.6 \%$

$12.4 \%$

$23.8 \%$

$2.2 \%$

$0.7 \%$

$0.3 \%$

$2.3 \%$

$2.7 \%$

$66.5 \%$

$100.0 \%$
0.85 [ 0.73, 0.99 ]

$0.76[0.54,1.07]$

$0.42[0.12,1.50]$

$0.95[0.60,1.50]$

$0.80[0.61,1.04$ ]

0.5 । $[0.05,5.45]$

$0.94[0.61,1.44]$

$0.25[0.06,1.15]$

$0.79[0.31,2.02]$

$0.67[0.12,3.78]$

$0.81[0.31,2.12]$

$1.25[0.83,1.86]$

$0.70[0.51,0.97]$

$0.81[0.29,2.28]$

$2.06[0.40,10.52]$

$2.00[0.20,20.33]$

$0.99[0.36,2.67]$

$0.84[0.36,1.96]$

$0.87[0.73,1.05]$

Total events: 254 (tacrolimus), 302 (cyclosporin)

Heterogeneity: $\mathrm{Chi}^{2}=11.33, \mathrm{df}=15(\mathrm{P}=0.73) ; \mathrm{I}^{2}=0.0 \%$

Test for overall effect: $Z=2.11(P=0.035)$ 
Analysis 2.2. Comparison 2 Stratified analysis, by cyclosporin formulation, Outcome 2 Graft loss.

Review: Cyclosporin versus tacrolimus for liver transplanted patients

Comparison: 2 Stratified analysis, by cyclosporin formulation

Outcome: 2 Graft loss

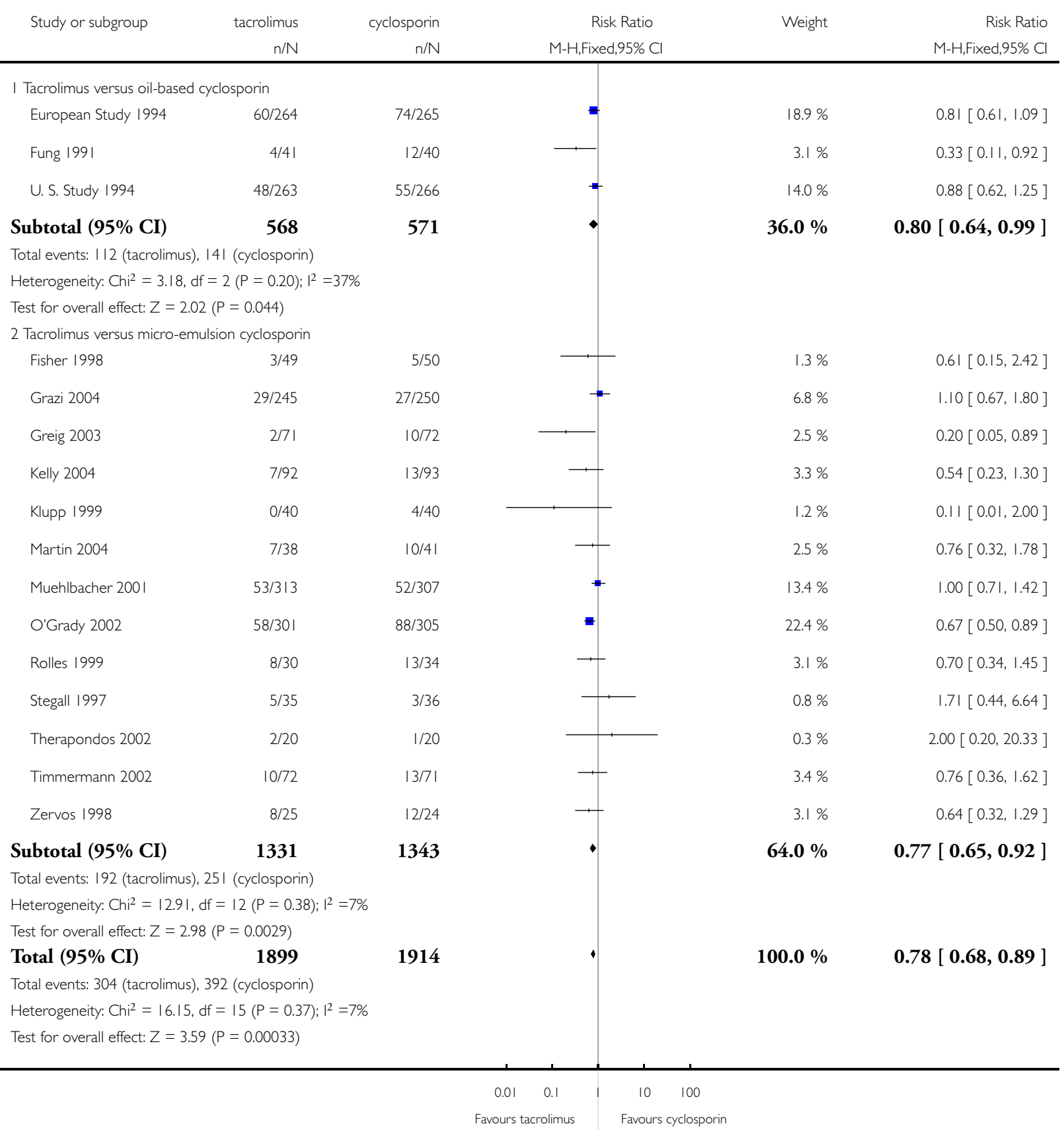


Analysis 2.3. Comparison 2 Stratified analysis, by cyclosporin formulation, Outcome 3 Acute rejection.

Review: Cyclosporin versus tacrolimus for liver transplanted patients

Comparison: 2 Stratified analysis, by cyclosporin formulation

Outcome: 3 Acute rejection

Study or subgroup $\quad$ tacrolimus $\quad$ cyclosporine $\quad$ Risk Ratio $\quad$ Weight

$\mathrm{n} / \mathrm{N} \quad \mathrm{n} / \mathrm{N} \quad \mathrm{M}-\mathrm{H}$, Fixed,95\% Cl M-H,Fixed,95\% Cl

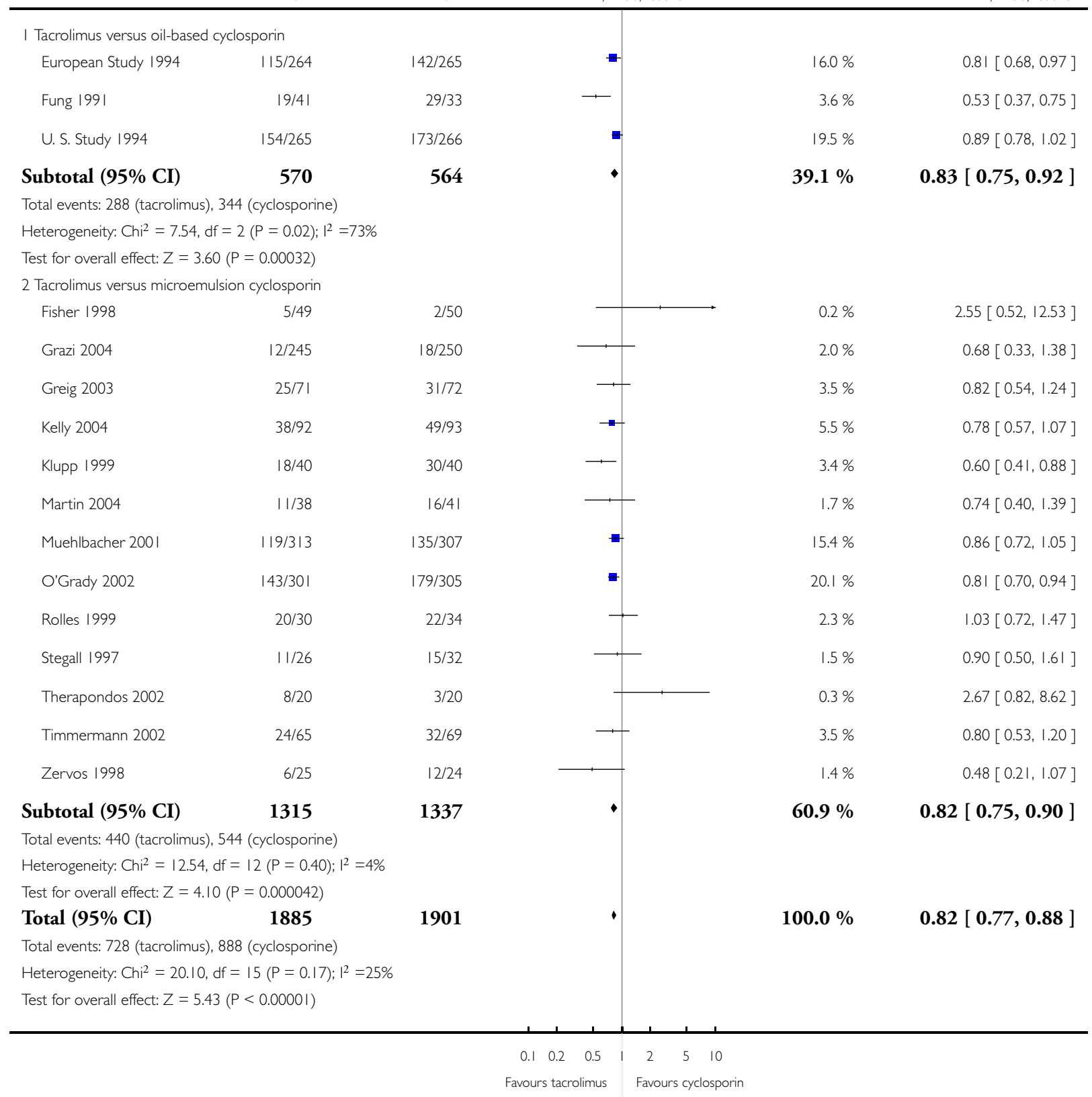

Cyclosporin versus tacrolimus for liver transplanted patients (Review) 
Analysis 2.4. Comparison 2 Stratified analysis, by cyclosporin formulation, Outcome 4 Steroid-resistent rejection.

Review: Cyclosporin versus tacrolimus for liver transplanted patients

Comparison: 2 Stratified analysis, by cyclosporin formulation

Outcome: 4 Steroid-resistent rejection

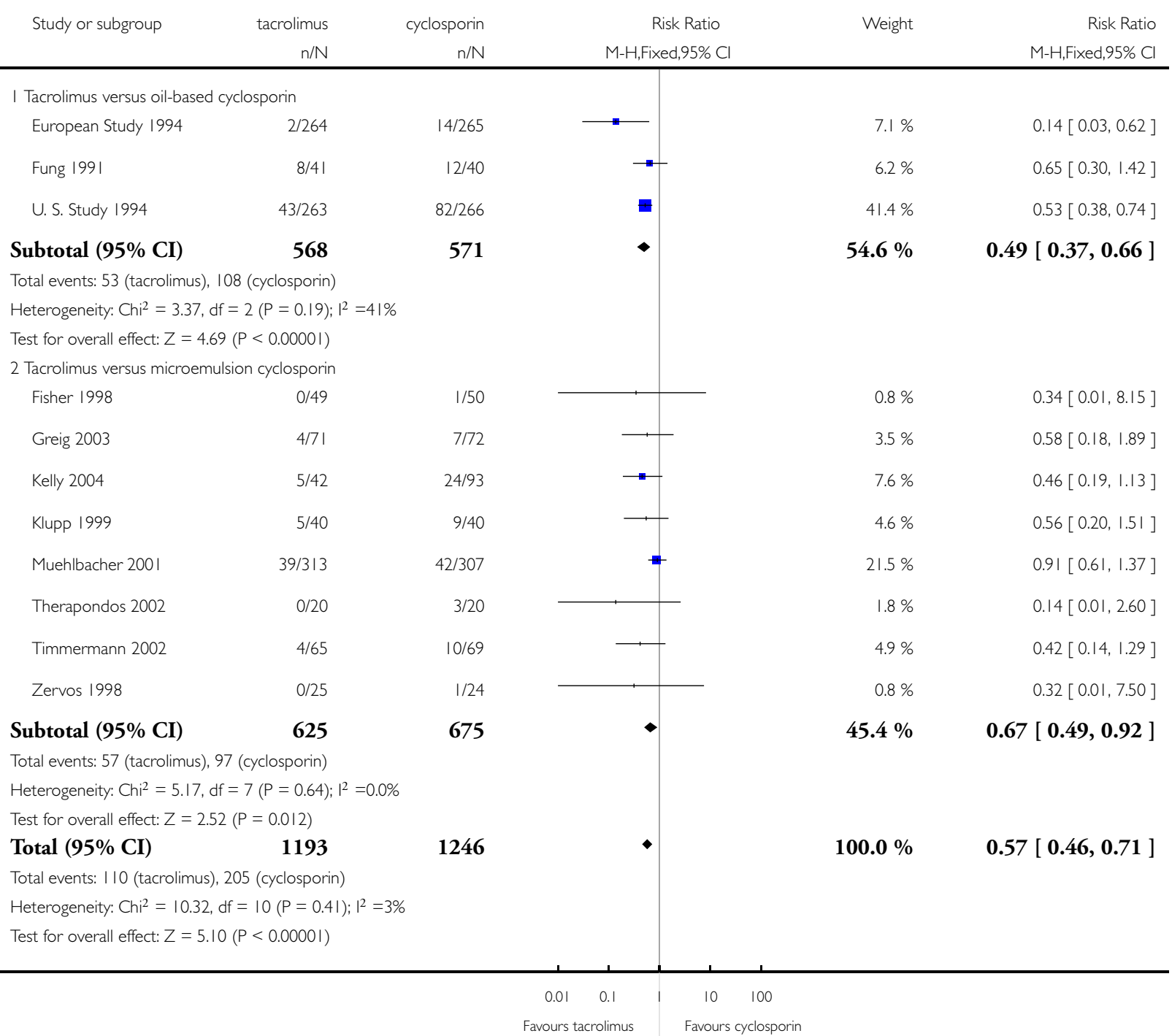


Analysis 2.5. Comparison 2 Stratified analysis, by cyclosporin formulation, Outcome 5 Dialysis (de-novo requirement post-transplantation).

Review: Cyclosporin versus tacrolimus for liver transplanted patients

Comparison: 2 Stratified analysis, by cyclosporin formulation

Outcome: 5 Dialysis (de-novo requirement post-transplantation)

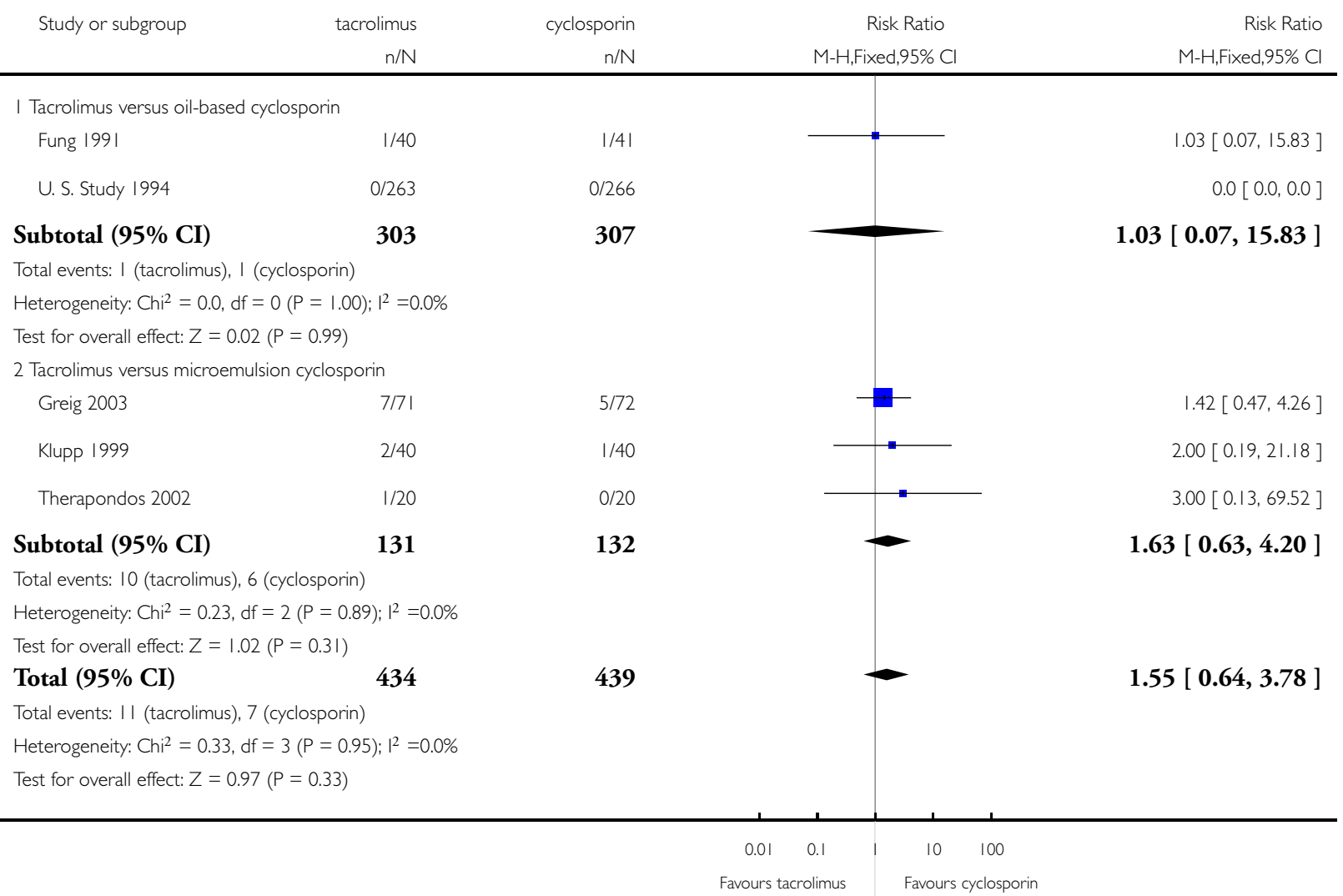


Analysis 2.6. Comparison 2 Stratified analysis, by cyclosporin formulation, Outcome 6 Diabetes mellitus: initially diagnosed after transplantation.

Review: Cyclosporin versus tacrolimus for liver transplanted patients

Comparison: 2 Stratified analysis, by cyclosporin formulation

Outcome: 6 Diabetes mellitus: initially diagnosed after transplantation

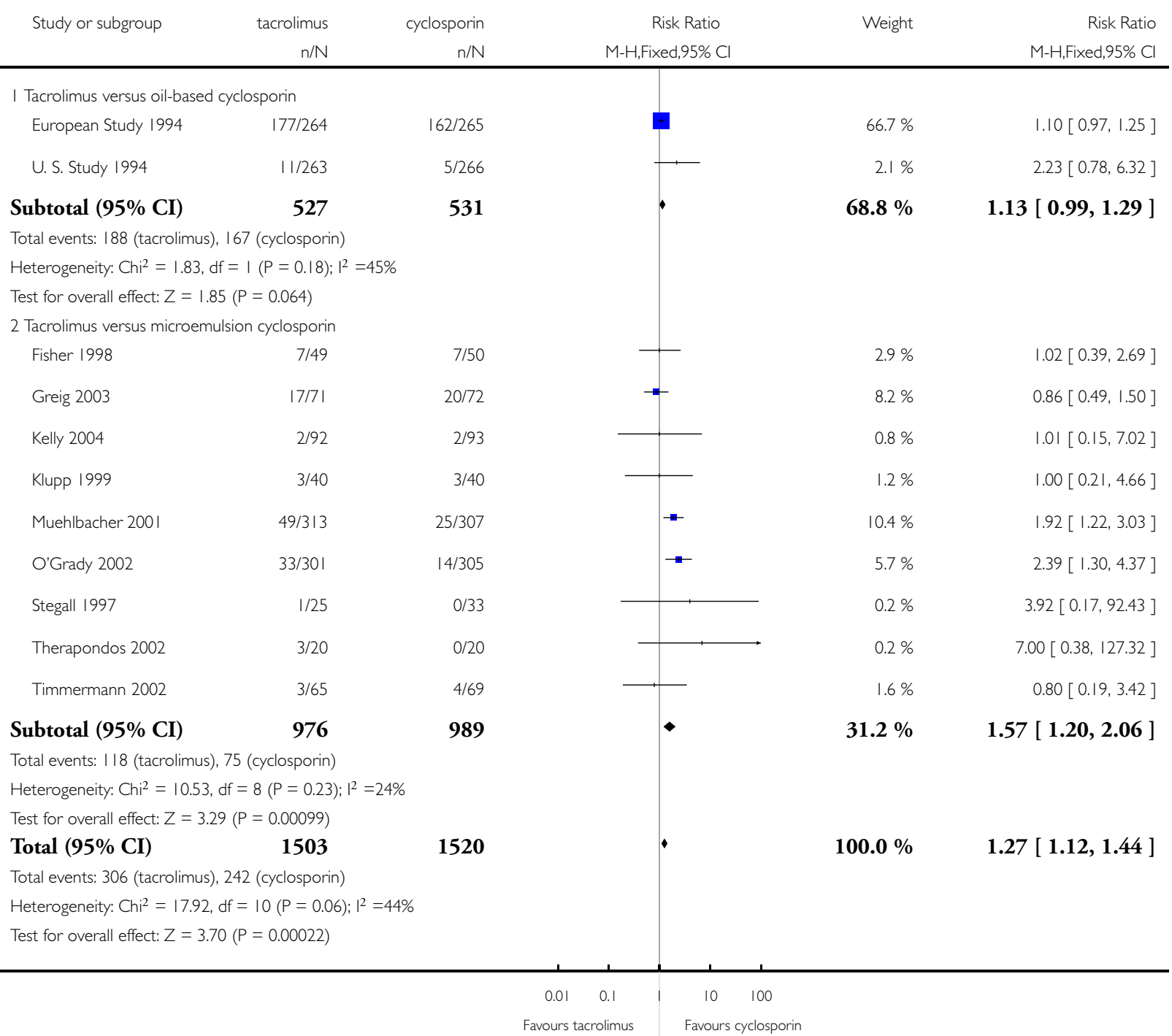


Analysis 2.7. Comparison 2 Stratified analysis, by cyclosporin formulation, Outcome 7 Post transplant lymphoproliferative disease.

Review: Cyclosporin versus tacrolimus for liver transplanted patients

Comparison: 2 Stratified analysis, by cyclosporin formulation

Outcome: 7 Post transplant lymphoproliferative disease

$\begin{array}{llll}\text { Study or subgroup } & \text { tacrolimus } & \text { Risk Ratio Ris Rorin }\end{array}$

$\mathrm{n} / \mathrm{N} \quad \mathrm{n} / \mathrm{N} \quad \mathrm{M}-\mathrm{H}$, Fixed,95\% Cl M-H,Fixed,95\% Cl

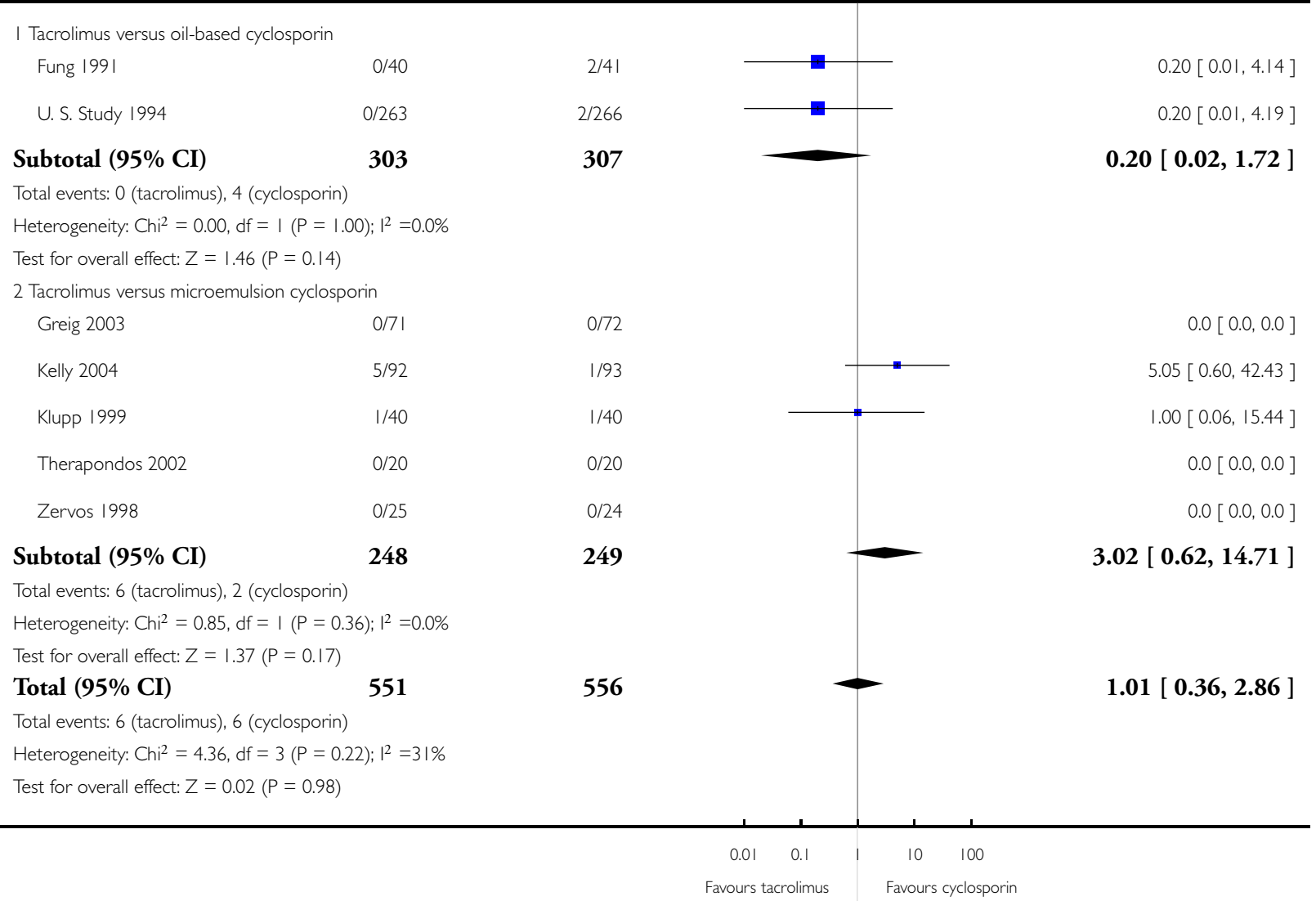


Analysis 2.8. Comparison 2 Stratified analysis, by cyclosporin formulation, Outcome 8 Patients withdrawn from tacrolimus or cyclosporin.

Review: Cyclosporin versus tacrolimus for liver transplanted patients

Comparison: 2 Stratified analysis, by cyclosporin formulation

Outcome: 8 Patients withdrawn from tacrolimus or cyclosporin

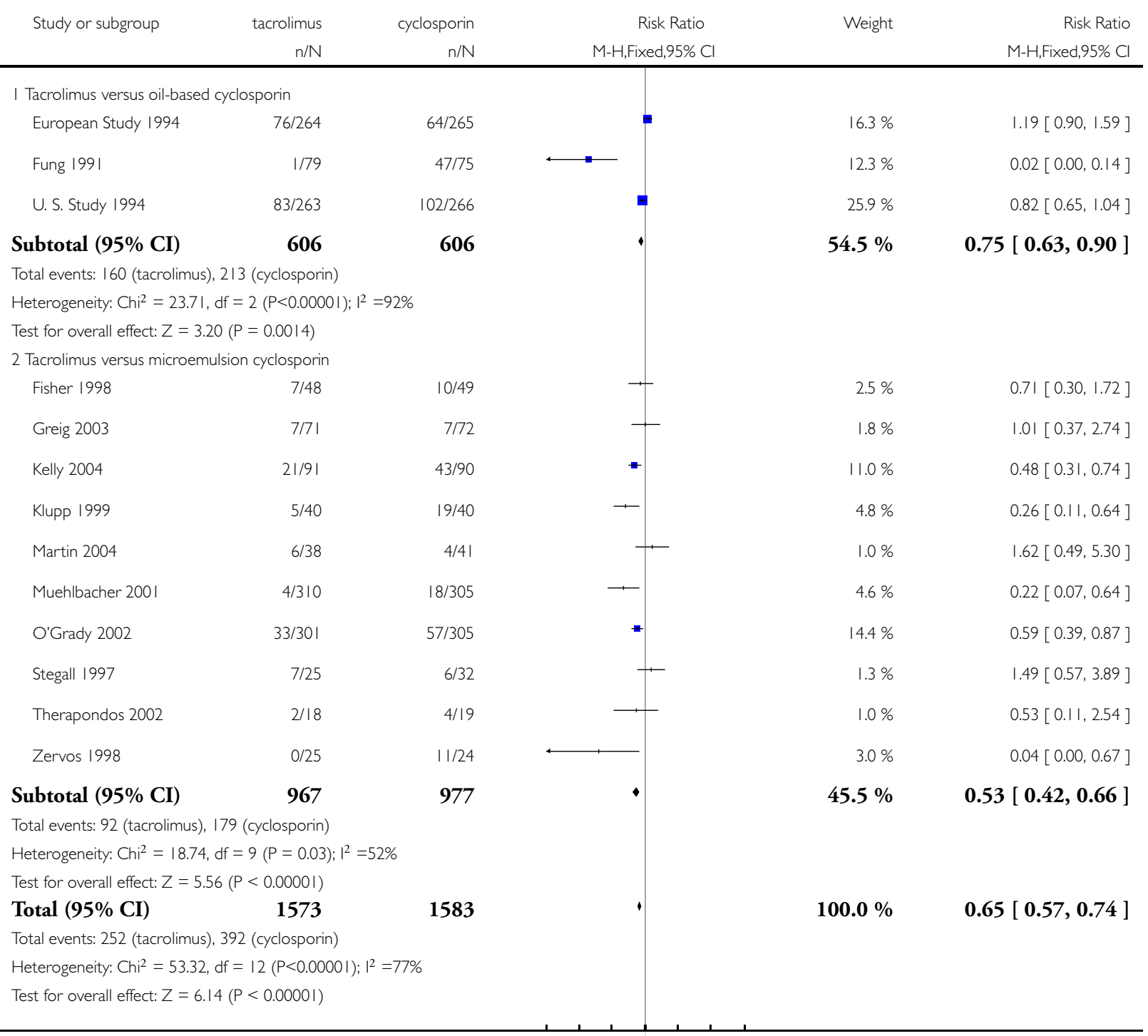

$0.0010 .01 \quad 0.1 \quad 1 \quad 10 \quad 100 \quad 1000$

Favours tacrolimus Favours cyclosporin 
Analysis 3.I. Comparison 3 Stratified analysis, by inclusion of children, Outcome I Mortality.

Review: Cyclosporin versus tacrolimus for liver transplanted patients

Comparison: 3 Stratified analysis, by inclusion of children

Outcome: | Mortality

tacrolimus

cyclosporin

Risk Ratio

Weight

Risk Ratio

I Studies of children or including children

Kelly 2004

$7 / 92$

$\mathrm{n} / \mathrm{N}$

M-H,Fixed,95\% Cl

M-H,Fixed,95\% Cl

U. S. Study 1994

9/93

Subtotal (95\% CI)

$31 / 263$

$33 / 266$

355

359

Total events: 38 (tacrolimus), 42 (cyclosporin)

Heterogeneity: $\mathrm{Chi}^{2}=0.12, \mathrm{df}=\mathrm{I}(\mathrm{P}=0.72) ; \mathrm{I}^{2}=0.0 \%$

Test for overall effect: $Z=0.42(P=0.67)$

2 Studies excluding children

$\begin{array}{lrr}\text { European Study 1994 } & 46 / 264 & 61 / 265 \\ \text { Fisher 1998 } & 1 / 49 & 2 / 50 \\ \text { Fung 199| } & 3 / 41 & 7 / 40 \\ \text { Grazi 2004 } & 34 / 245 & 37 / 250 \\ \text { Greig 2003 } & 2 / 7 \mid & 8 / 72 \\ \text { Klupp 1999 } & 2 / 40 & 3 / 40 \\ \text { Martin 2004 } & 6 / 38 & 8 / 4 \\ \text { Muehlbacher 200| } & 47 / 313 & 37 / 307 \\ \text { O'Grady 2002 } & 50 / 301 & 72 / 305 \\ \text { Rolles 1999 } & 5 / 30 & 7 / 34 \\ \text { Stegall 1997 } & 4 / 35 & 2 / 36 \\ \text { Therapondos 2002 } & 2 / 20 & 1 / 20 \\ \text { Timmermann 2002 } & 7 / 72 & \mathbf{1 5 5} \\ \text { Zervos 1998 } & 7 / 25 & 8 / 24 \\ \text { Subtotal (95\% CI) } & \mathbf{1 5 4 4} & \end{array}$

Subtotal (95\% CI)

1544

1555

Total events: 216 (tacrolimus), 260 (cyclosporin)

Heterogeneity: $\mathrm{Chi}^{2}=11.05, \mathrm{df}=13(\mathrm{P}=0.61) ;\left.\right|^{2}=0.0 \%$

Test for overall effect: $Z=2.11(P=0.035)$

Total (95\% CI)

1899

1914

$+$

$3.0 \%$

$0.79[0.31,2.02]$

$10.9 \%$

$0.95[0.60,1.50]$

$13.9 \%$

$0.91[0.61,1.38$ ]

Total events: 254 (tacrolimus), 302 (cyclosporin)

Heterogeneity: Chi $^{2}=11.33, \mathrm{df}=15(\mathrm{P}=0.73) ;\left.\right|^{2}=0.0 \%$

Test for overall effect: $Z=2.11(P=0.035)$

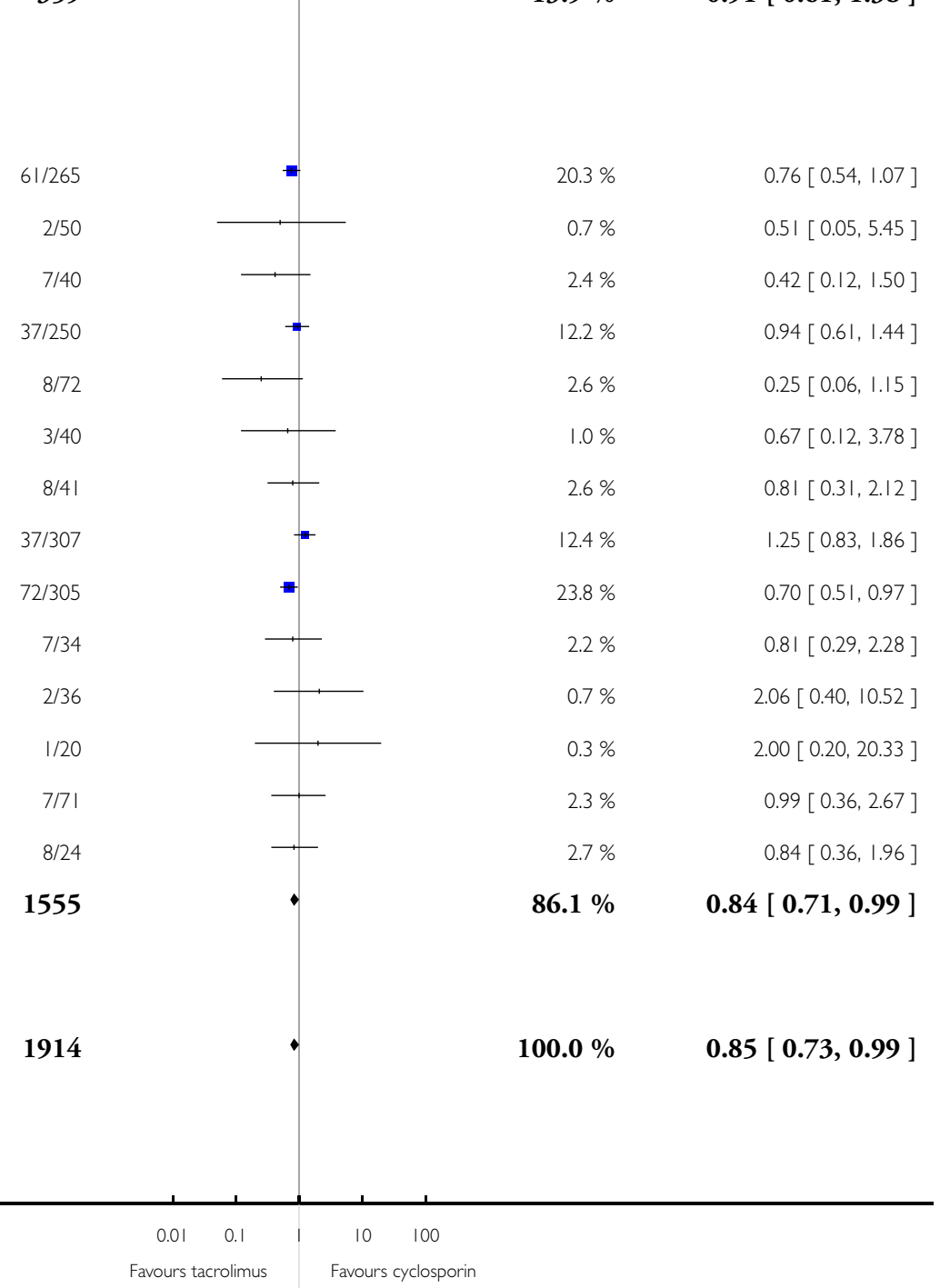

Cyclosporin versus tacrolimus for liver transplanted patients (Review) 
Analysis 3.2. Comparison 3 Stratified analysis, by inclusion of children, Outcome 2 Graft loss.

Review: Cyclosporin versus tacrolimus for liver transplanted patients

Comparison: 3 Stratified analysis, by inclusion of children

Outcome: 2 Graft loss

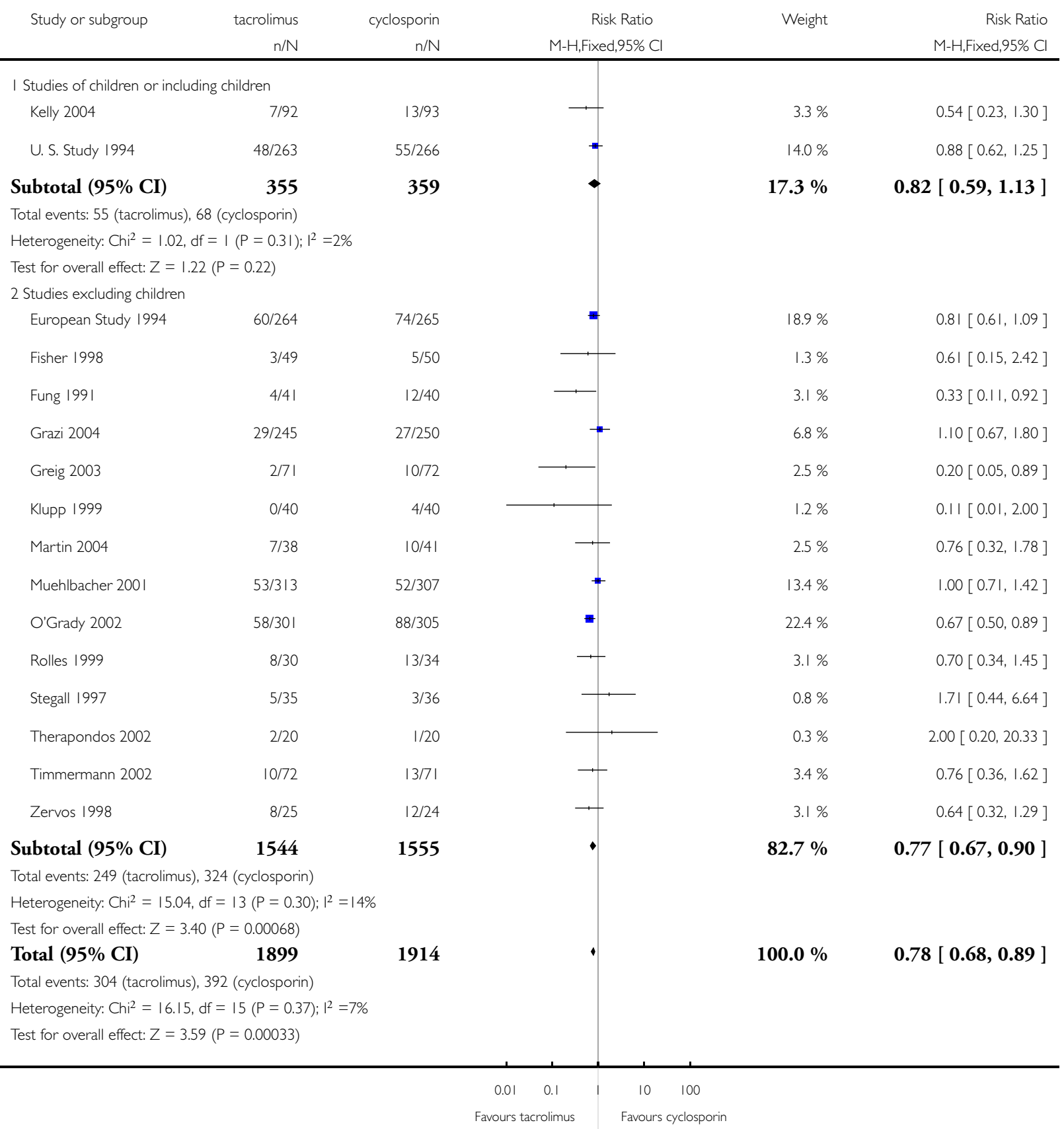


Analysis 3.3. Comparison 3 Stratified analysis, by inclusion of children, Outcome 3 Acute rejection.

Review: Cyclosporin versus tacrolimus for liver transplanted patients

Comparison: 3 Stratified analysis, by inclusion of children

Outcome: 3 Acute rejection

Study or subgroup $\quad$ tacrolimus $\quad$ cyclosporin $\quad$ Risk Ratio $\quad$ Weight

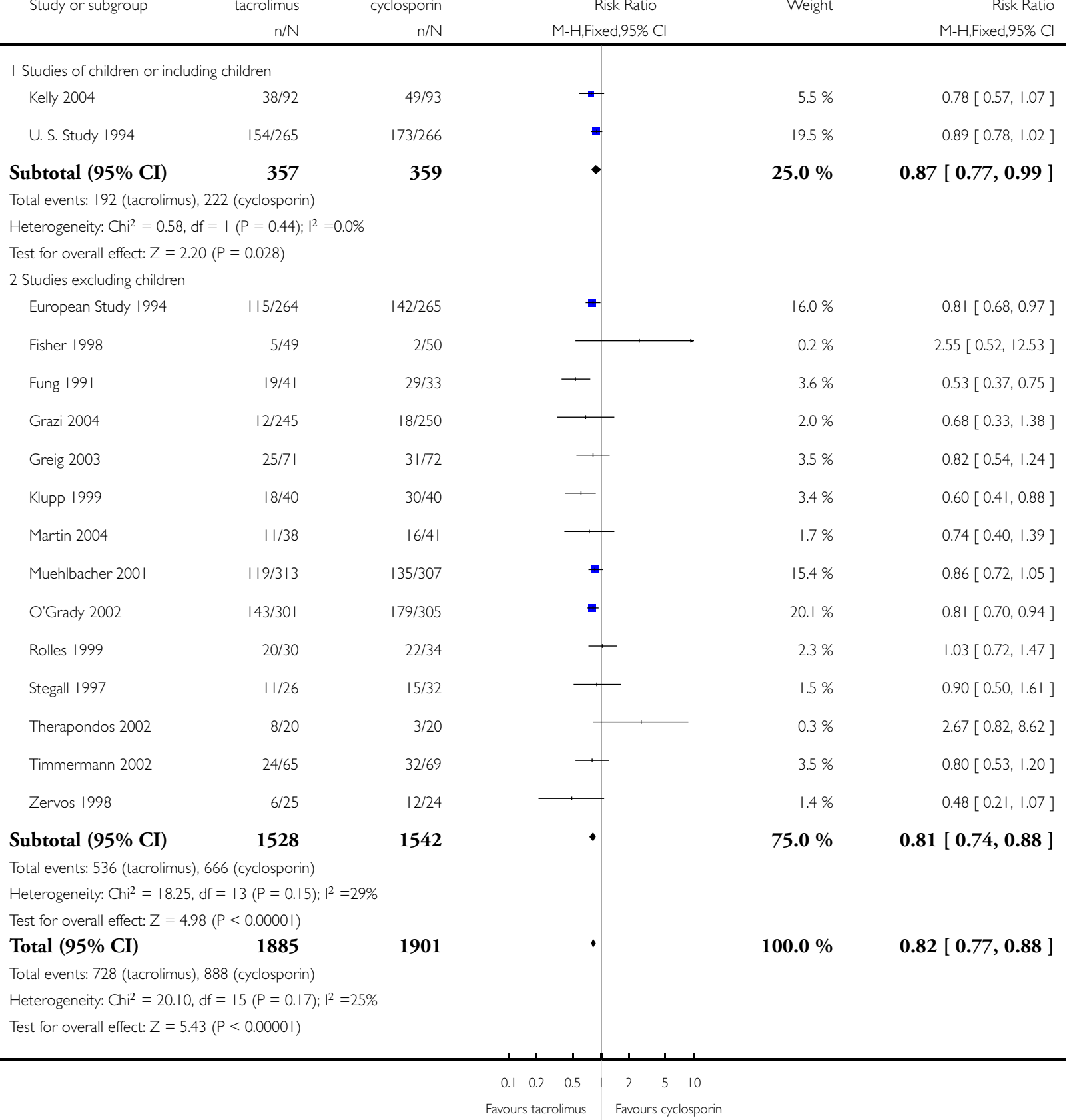

Cyclosporin versus tacrolimus for liver transplanted patients (Review) 
Analysis 3.4. Comparison 3 Stratified analysis, by inclusion of children, Outcome 4 Steroid-resistent rejection.

Review: Cyclosporin versus tacrolimus for liver transplanted patients

Comparison: 3 Stratified analysis, by inclusion of children

Outcome: 4 Steroid-resistent rejection

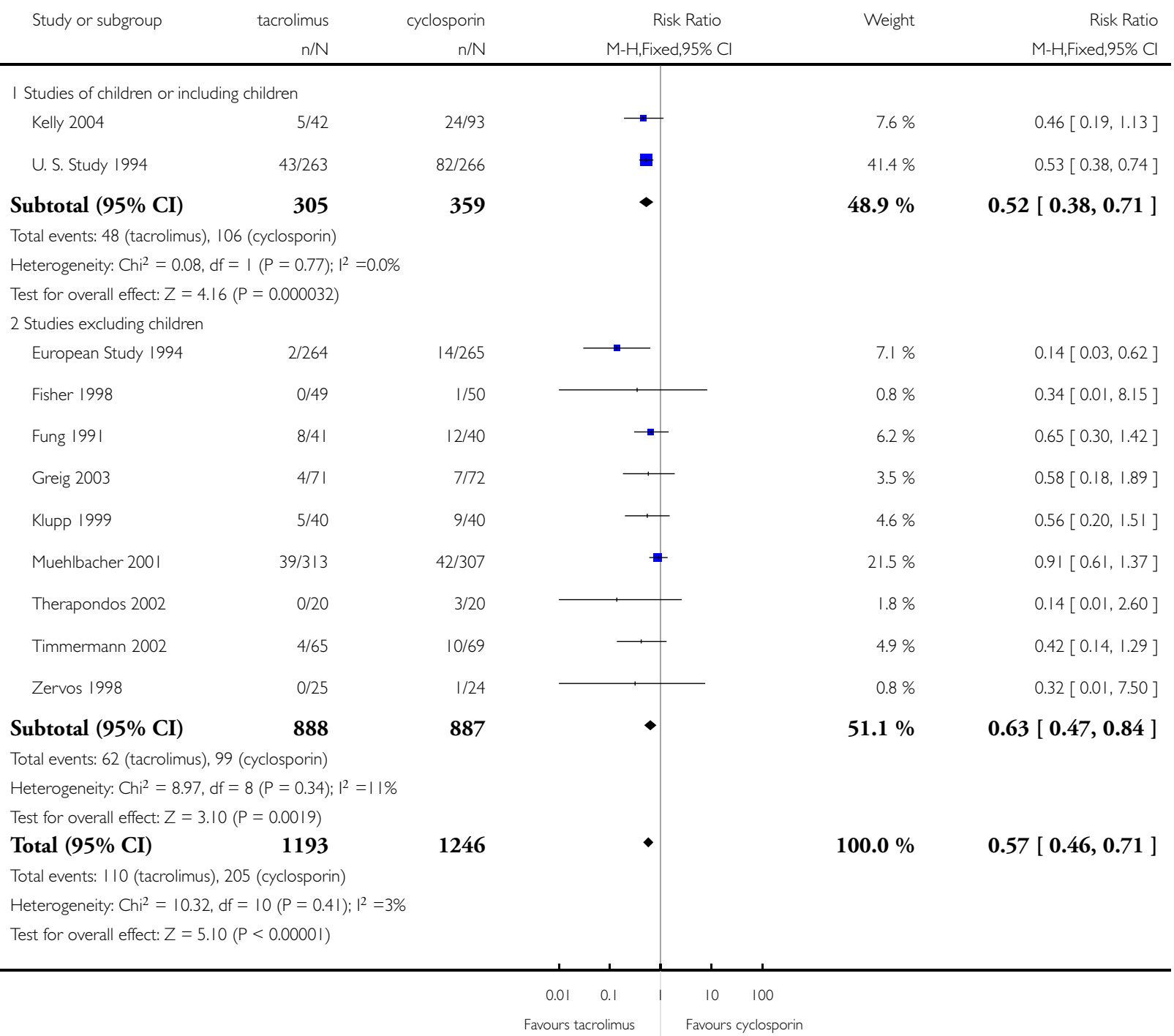


Analysis 3.5. Comparison 3 Stratified analysis, by inclusion of children, Outcome 5 Dialysis (de-novo requirement post-transplantation).

Review: Cyclosporin versus tacrolimus for liver transplanted patients

Comparison: 3 Stratified analysis, by inclusion of children

Outcome: 5 Dialysis (de-novo requirement post-transplantation)

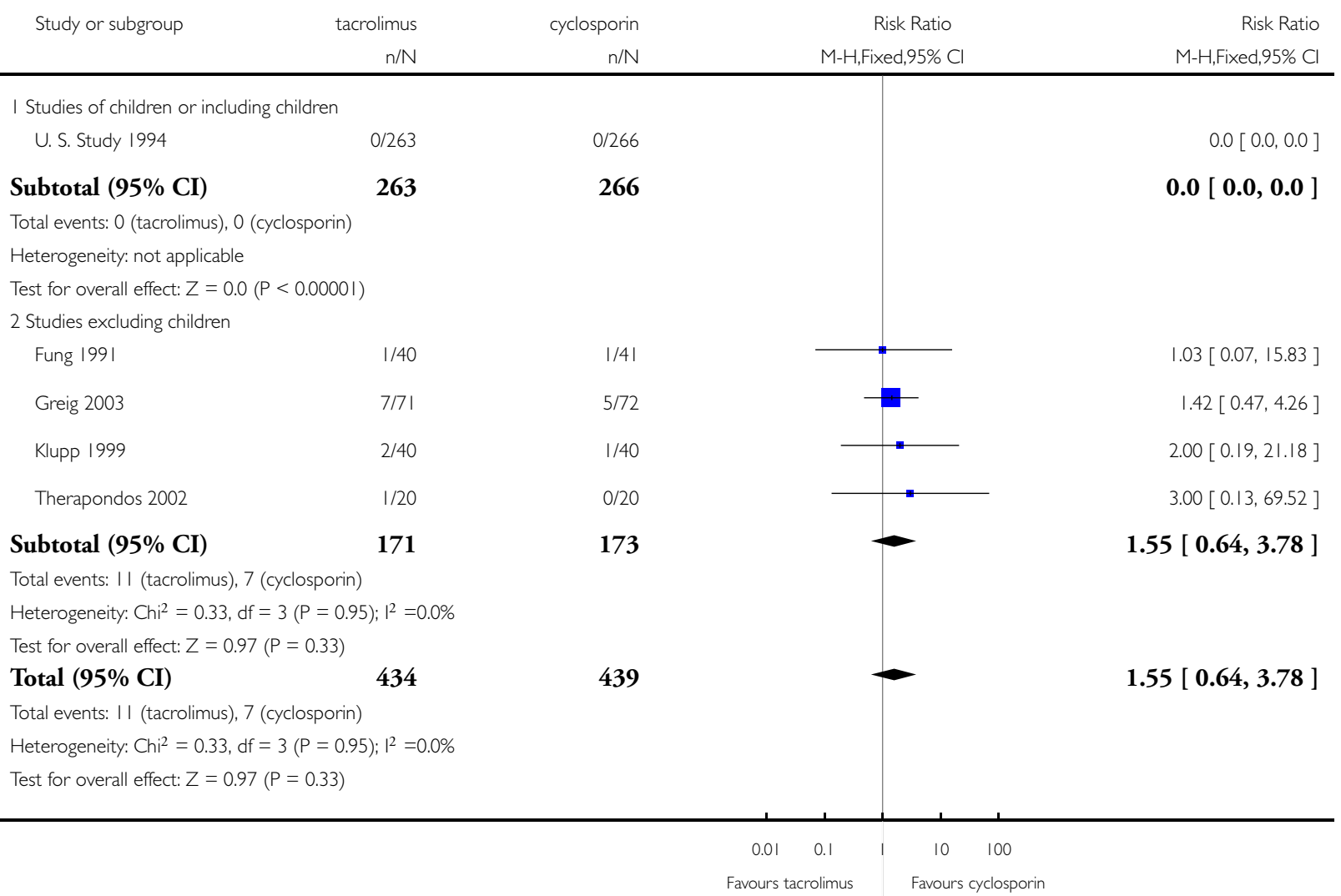


Analysis 3.6. Comparison 3 Stratified analysis, by inclusion of children, Outcome 6 Diabetes mellitus: initially diagnosed after transplantation.

Review: Cyclosporin versus tacrolimus for liver transplanted patients
Comparison: 3 Stratified analysis, by inclusion of children
Outcome: 6 Diabetes mellitus: initially diagnosed after transplantation

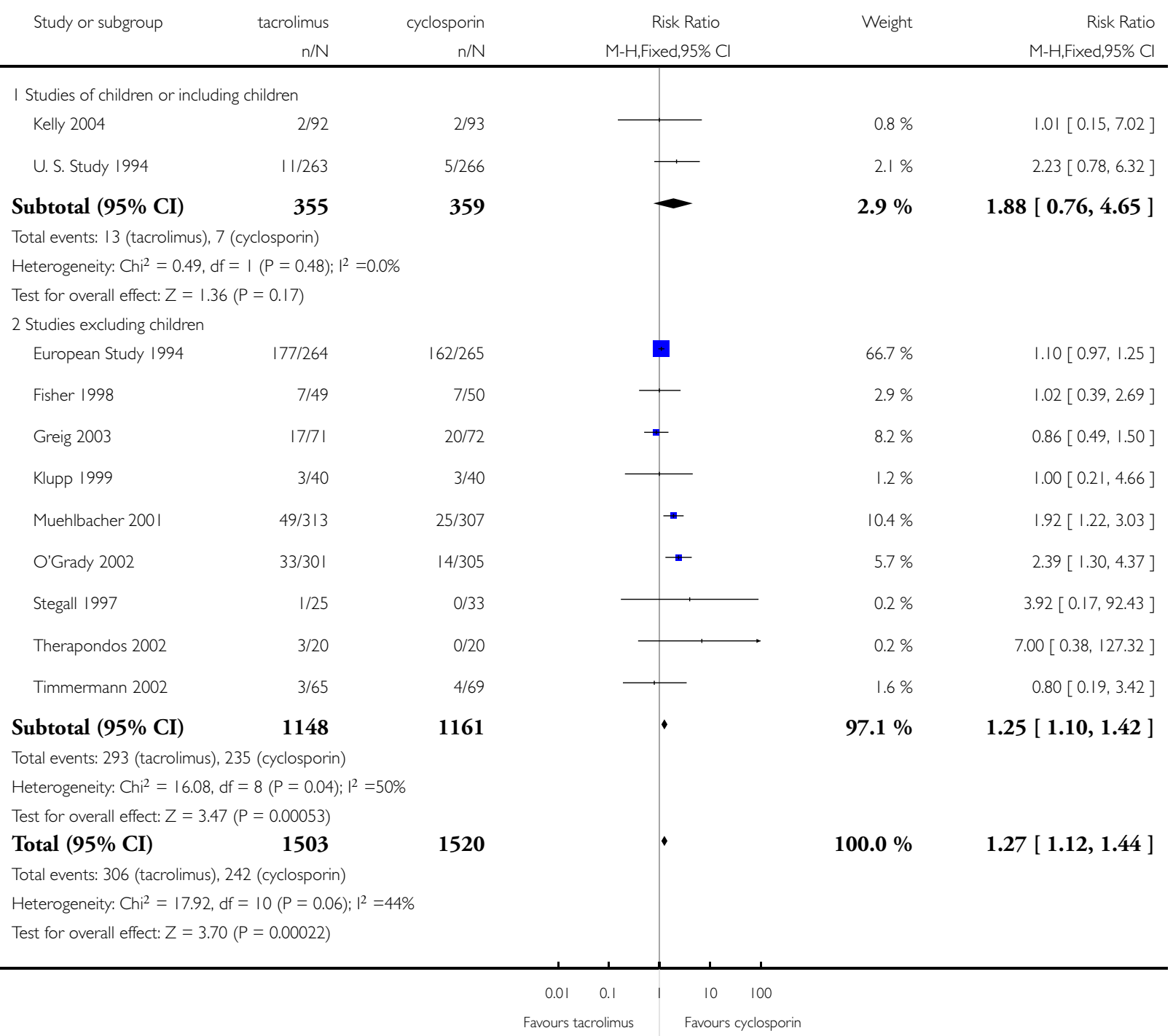


Analysis 3.7. Comparison 3 Stratified analysis, by inclusion of children, Outcome 7 Post transplant lymphoproliferative disease.

Review: Cyclosporin versus tacrolimus for liver transplanted patients

Comparison: 3 Stratified analysis, by inclusion of children

Outcome: 7 Post transplant lymphoproliferative disease

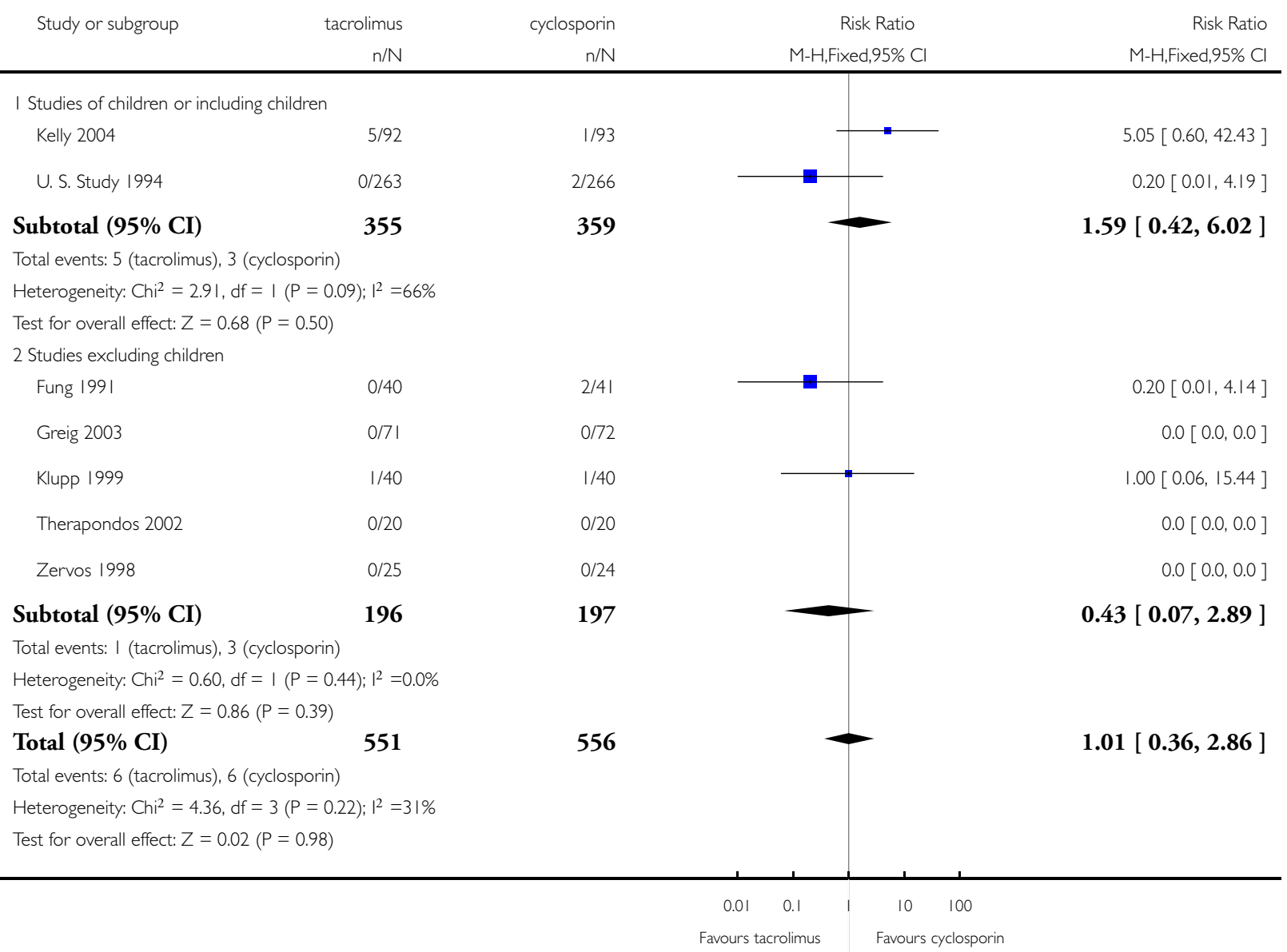


Analysis 3.8. Comparison 3 Stratified analysis, by inclusion of children, Outcome 8 Patients withdrawn from tacrolimus or cyclosporin.

Review: Cyclosporin versus tacrolimus for liver transplanted patients

Comparison: 3 Stratified analysis, by inclusion of children

Outcome: 8 Patients withdrawn from tacrolimus or cyclosporin

\begin{tabular}{|c|c|c|c|c|c|}
\hline \multirow[t]{2}{*}{ Study or subgroup } & tacrolimus & cyclosporin & Risk Ratio & Weight & Risk Ratio \\
\hline & $\mathrm{n} / \mathrm{N}$ & $\mathrm{n} / \mathrm{N}$ & M-H,Fixed,95\% Cl & & $\mathrm{M}-\mathrm{H}$,Fixed,95\% Cl \\
\hline \multicolumn{6}{|c|}{ I Studies of children or including children } \\
\hline Kelly 2004 & $2|/ 9|$ & $43 / 90$ & 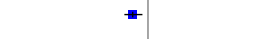 & $11.0 \%$ & $0.48[0.31,0.74]$ \\
\hline U. S. Study 1994 & $83 / 263$ & $102 / 266$ & H & $25.9 \%$ & $0.82[0.65,1.04]$ \\
\hline Subtotal (95\% CI) & 354 & 356 & - & $36.9 \%$ & $0.72[0.59,0.89]$ \\
\hline \multicolumn{6}{|c|}{ Total events: I04 (tacrolimus), I 45 (cyclosporin) } \\
\hline \multicolumn{6}{|c|}{ Heterogeneity: $\mathrm{Chi}^{2}=4.5 \mathrm{I}, \mathrm{df}=\mathrm{I}(\mathrm{P}=0.03) ; \mathrm{I}^{2}=78 \%$} \\
\hline \multicolumn{6}{|c|}{ Test for overall effect: $Z=3.12(P=0.0018)$} \\
\hline \multicolumn{6}{|l|}{2 Studies excluding children } \\
\hline European Study 1994 & $76 / 264$ & $64 / 265$ & P & $16.3 \%$ & $1.19[0.90,1.59]$ \\
\hline Fisher 1998 & $7 / 48$ & $10 / 49$ & $\rightarrow$ & $2.5 \%$ & $0.71[0.30,1.72]$ \\
\hline Fung 1991 & $1 / 79$ & $47 / 75$ & $\longleftarrow$ & $12.3 \%$ & $0.02[0.00,0.14]$ \\
\hline Greig 2003 & $7 / 71$ & $7 / 72$ & 1 & $1.8 \%$ & $1.01[0.37,2.74]$ \\
\hline Klupp 1999 & $5 / 40$ & $19 / 40$ & + & $4.8 \%$ & $0.26[0.11,0.64]$ \\
\hline Martin 2004 & $6 / 38$ & $4 / 41$ & † & $1.0 \%$ & $1.62[0.49,5.30]$ \\
\hline Muehlbacher 2001 & $4 / 310$ & $18 / 305$ & $\longrightarrow$ & $4.6 \%$ & $0.22[0.07,0.64]$ \\
\hline O'Grady 2002 & $33 / 301$ & $57 / 305$ & 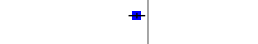 & $14.4 \%$ & $0.59[0.39,0.87]$ \\
\hline Stegall 1997 & $7 / 25$ & $6 / 32$ & - & $1.3 \%$ & $1.49[0.57,3.89]$ \\
\hline Therapondos 2002 & $2 / 18$ & $4 / 19$ & $\longrightarrow$ & $1.0 \%$ & $0.53[0.11,2.54]$ \\
\hline Zervos 1998 & $0 / 25$ & $11 / 24$ & - & $3.0 \%$ & $0.04[0.00,0.67]$ \\
\hline Subtotal (95\% CI) & 1219 & 1227 & - & $63.1 \%$ & $0.61[0.50,0.73]$ \\
\hline \multicolumn{6}{|c|}{ Total events: I 48 (tacrolimus), 247 (cyclosporin) } \\
\hline \multicolumn{6}{|c|}{ Heterogeneity: $\mathrm{Chi}^{2}=50.89, \mathrm{df}=10(\mathrm{P}<0.0000 \mathrm{I}) ; \mathrm{I}^{2}=80 \%$} \\
\hline \multicolumn{6}{|c|}{ Test for overall effect: $Z=5.30(P<0.0000 I)$} \\
\hline Total (95\% CI) & 1573 & 1583 & $\cdot$ & $100.0 \%$ & $0.65[0.57,0.74]$ \\
\hline \multicolumn{6}{|c|}{ Total events: 252 (tacrolimus), 392 (cyclosporin) } \\
\hline \multicolumn{6}{|c|}{ Heterogeneity: $\mathrm{Ch}^{2}=53.32, \mathrm{df}=12(\mathrm{P}<0.0000 \mathrm{I}) ; \mathrm{I}^{2}=77 \%$} \\
\hline Test for overall effect: $Z=$ & $<0.00001)$ & & & & \\
\hline
\end{tabular}

$0.0010 .01 \quad 0.1 \quad 1 \quad 10 \quad 100 \quad 1000$

Favours tacrolimus Favours cyclosporin 
Analysis 4.I. Comparison 4 Stratified analysis, by studies reporting 12 month data, Outcome I Mortality.

Review: Cyclosporin versus tacrolimus for liver transplanted patients

Comparison: 4 Stratified analysis, by studies reporting 12 month data

Outcome: | Mortality

tacrolimus

cyclosporin

Risk Ratio

Weight

Risk Ratio

$\mathrm{n} / \mathrm{N}$

$n / N$

M-H,Fixed,95\% Cl

M-H,Fixed,95\% Cl

I Studies reporting less than 12 month data

Stegall 1997

$4 / 35$

$2 / 36$

Timmermann 2002

$7 / 72$

$7 / 71$

Subtotal (95\% CI)

107

107

Total events: I I (tacrolimus), 9 (cyclosporin)

Heterogeneity: Chi $^{2}=0.57, \mathrm{df}=\mathrm{I}(\mathrm{P}=0.45) ;\left.\right|^{2}=0.0 \%$

Test for overall effect: $Z=0.47(P=0.64)$

2 Studies reporting 12 month data

\begin{tabular}{|c|c|}
\hline European Study 1994 & $46 / 264$ \\
\hline Fisher 1998 & $1 / 49$ \\
\hline Fung 1991 & $3 / 41$ \\
\hline Grazi 2004 & $34 / 245$ \\
\hline Greig 2003 & $2 / 71$ \\
\hline Kelly 2004 & $7 / 92$ \\
\hline Klupp 1999 & $2 / 40$ \\
\hline Martin 2004 & $6 / 38$ \\
\hline Muehlbacher 2001 & $47 / 313$ \\
\hline O'Grady 2002 & $50 / 301$ \\
\hline Rolles 1999 & $5 / 30$ \\
\hline Therapondos 2002 & $2 / 20$ \\
\hline U. S. Study 1994 & $31 / 263$ \\
\hline Zervos 1998 & $7 / 25$ \\
\hline
\end{tabular}

Subtotal (95\% CI)

1792

1807

Total events: 243 (tacrolimus), 293 (cyclosporin)

Heterogeneity: $\mathrm{Chi}^{2}=10.09, \mathrm{df}=13(\mathrm{P}=0.69) ;\left.\right|^{2}=0.0 \%$

Test for overall effect: $Z=2.25(P=0.025)$

Total (95\% CI)

1899

1914

\begin{tabular}{l|l} 
& \\
\hline & \\
\hline
\end{tabular}

$0.7 \%$

$2.06[0.40,10.52]$

$2.3 \%$

$0.99[0.36,2.67]$

$3.0 \%$

$1.22[0.53,2.82]$

Total events: 254 (tacrolimus), 302 (cyclosporin)

Heterogeneity: Chi $^{2}=11.33, \mathrm{df}=15(\mathrm{P}=0.73) ;\left.\right|^{2}=0.0 \%$

Test for overall effect: $Z=2.11(P=0.035)$

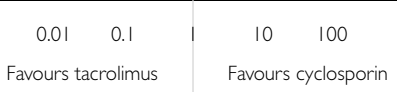

$20.3 \%$

$0.7 \%$

$2.4 \%$

$12.2 \%$

$2.6 \%$

$3.0 \%$

$1.0 \%$

$2.6 \%$

$12.4 \%$

$23.8 \%$

$2.2 \%$

$0.3 \%$

$10.9 \%$

$2.7 \%$

$97.0 \%$

$100.0 \%$

$0.85[0.73,0.99]$ 
Analysis 4.2. Comparison 4 Stratified analysis, by studies reporting 12 month data, Outcome 2 Graft loss.

Review: Cyclosporin versus tacrolimus for liver transplanted patients

Comparison: 4 Stratified analysis, by studies reporting 12 month data

Outcome: 2 Graft loss

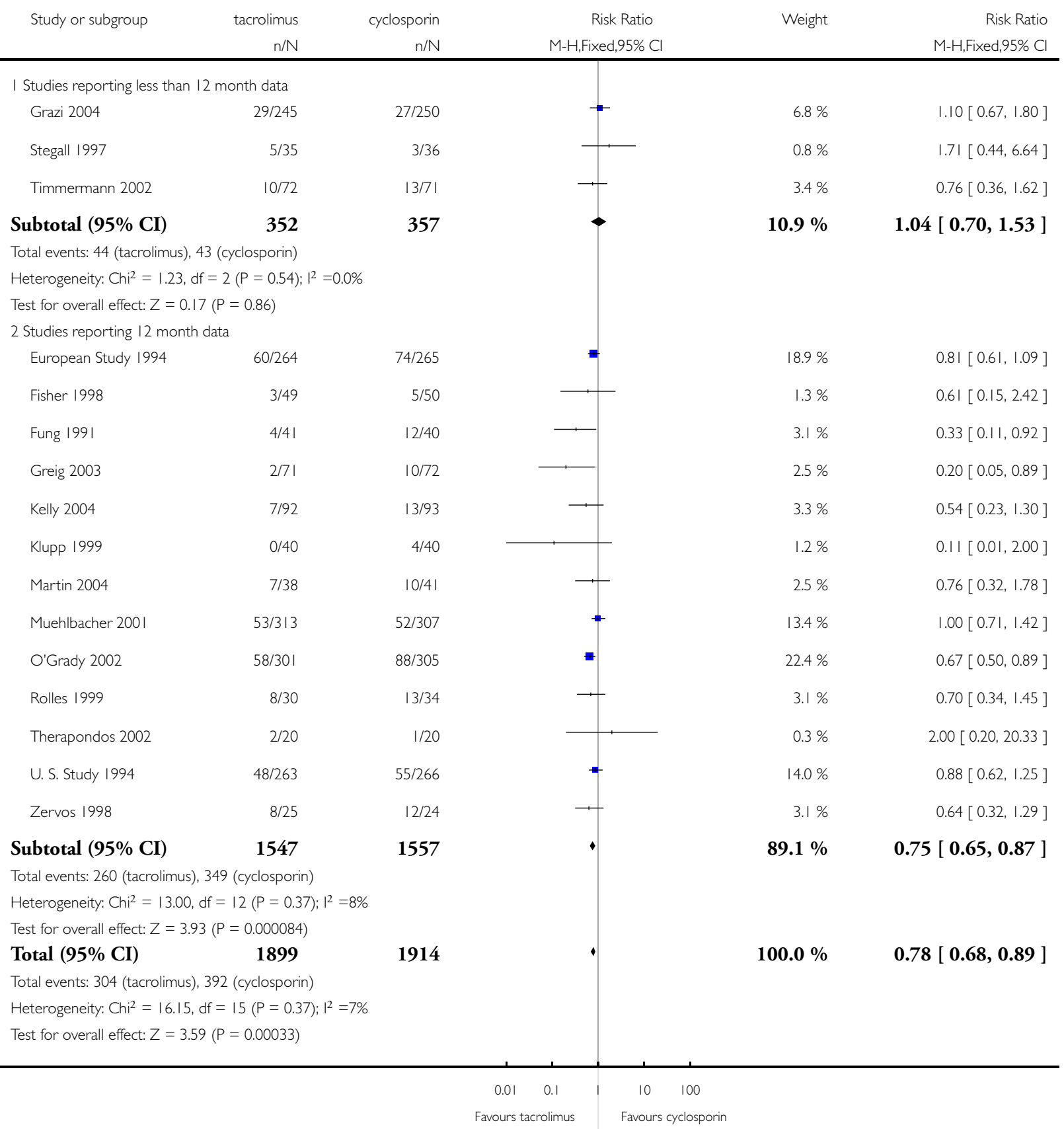


Analysis 4.3. Comparison 4 Stratified analysis, by studies reporting 12 month data, Outcome 3 Acute rejection.

Review: Cyclosporin versus tacrolimus for liver transplanted patients
Comparison: 4 Stratified analysis, by studies reporting 12 month data
Outcome: 3 Acute rejection

\begin{tabular}{|c|c|c|c|c|c|}
\hline \multirow[t]{2}{*}{ Study or subgroup } & tacrolimus & cyclosporin & \multirow{2}{*}{$\begin{array}{c}\text { Risk Ratio } \\
\text { M-H,Fixed,95\% Cl }\end{array}$} & \multirow[t]{2}{*}{ Weight } & \multirow{2}{*}{$\begin{array}{r}\text { Risk Ratic } \\
\text { M-H,Fixed,95\% C }\end{array}$} \\
\hline & $\mathrm{n} / \mathrm{N}$ & $\mathrm{n} / \mathrm{N}$ & & & \\
\hline \multicolumn{6}{|c|}{ I Studies reporting less than 12 month data } \\
\hline Stegall 1997 & $11 / 26$ & $15 / 32$ & $\longrightarrow$ & $1.5 \%$ & $0.90[0.50,1.61]$ \\
\hline Timmermann 2002 & $24 / 65$ & $32 / 69$ & $\rightarrow$ & $3.5 \%$ & $0.80[0.53,1.20]$ \\
\hline Subtotal $(95 \% \mathrm{CI})$ & 91 & 101 & 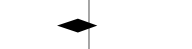 & $5.0 \%$ & $0.83[0.59,1.16]$ \\
\hline \multicolumn{6}{|c|}{ Total events: 35 (tacrolimus), 47 (cyclosporin) } \\
\hline \multicolumn{6}{|c|}{ Heterogeneity: $\mathrm{Chi}^{2}=0.12, \mathrm{df}=\mathrm{I}(\mathrm{P}=0.73) ;\left.\right|^{2}=0.0 \%$} \\
\hline \multicolumn{6}{|c|}{ Test for overall effect: $Z=1.11(P=0.27)$} \\
\hline \multicolumn{6}{|c|}{2 Studies reporting 12 month data } \\
\hline European Study 1994 & $115 / 264$ & $142 / 265$ & \# & $16.0 \%$ & $0.81[0.68,0.97]$ \\
\hline Fisher 1998 & $5 / 49$ & $2 / 50$ & + & $0.2 \%$ & $2.55[0.52,12.53]$ \\
\hline Fung |99| & $|9 / 4|$ & $29 / 33$ & $\longrightarrow$ & $3.6 \%$ & $0.53[0.37,0.75]$ \\
\hline Grazi 2004 & $12 / 245$ & $18 / 250$ & + & $2.0 \%$ & $0.68[0.33,1.38]$ \\
\hline Greig 2003 & $25 / 7 \mid$ & $31 / 72$ & $\rightarrow$ & $3.5 \%$ & $0.82[0.54,1.24]$ \\
\hline Kelly 2004 & $38 / 92$ & $49 / 93$ & $\rightarrow$ & $5.5 \%$ & $0.78[0.57,1.07]$ \\
\hline Klupp 1999 & $18 / 40$ & $30 / 40$ & $一$ & $3.4 \%$ & $0.60[0.41,0.88]$ \\
\hline Martin 2004 & $11 / 38$ & $16 / 4 \mid$ & \begin{tabular}{l|l}
$\cdot$ & \\
$\cdot$ &
\end{tabular} & $1.7 \%$ & $0.74[0.40,1.39]$ \\
\hline Muehlbacher 2001 & $119 / 313$ & 135/307 & $=$ & $15.4 \%$ & $0.86[0.72,1.05]$ \\
\hline O'Grady 2002 & $|43 / 30|$ & |79/305 & - & $20.1 \%$ & $0.81[0.70,0.94]$ \\
\hline Rolles 1999 & $20 / 30$ & $22 / 34$ & + & $2.3 \%$ & $1.03[0.72,1.47]$ \\
\hline Therapondos 2002 & $8 / 20$ & $3 / 20$ & & $0.3 \%$ & $2.67[0.82,8.62]$ \\
\hline U. S. Study 1994 & I54/265 & $173 / 266$ & $\#$ & $19.5 \%$ & $0.89[0.78,1.02]$ \\
\hline Zervos 1998 & $6 / 25$ & $12 / 24$ & 7 & $1.4 \%$ & $0.48[0.21,1.07]$ \\
\hline Subtotal (95\% CI) & 1794 & 1800 & - & $95.0 \%$ & $0.82[0.77,0.89]$ \\
\hline \multicolumn{6}{|c|}{ Total events: 693 (tacrolimus), 84I (cyclosporin) } \\
\hline \multicolumn{6}{|c|}{ Heterogeneity: $\mathrm{Chi}^{2}=19.97, \mathrm{df}=13(\mathrm{P}=0.10) ; \mathrm{I}^{2}=35 \%$} \\
\hline \multicolumn{6}{|c|}{ Test for overall effect: $Z=5.32(P<0.0000 I)$} \\
\hline Total $(95 \%$ CI $)$ & 1885 & 1901 & - & $100.0 \%$ & $0.82[0.77,0.88]$ \\
\hline \multicolumn{6}{|c|}{ Total events: 728 (tacrolimus), 888 (cyclosporin) } \\
\hline \multicolumn{6}{|c|}{ Heterogeneity: $\mathrm{Chi}^{2}=20.10, \mathrm{df}=15(P=0.17) ;\left.\right|^{2}=25 \%$} \\
\hline Test for overall effect: $Z=$ & $<0.0000$ I) & & & & \\
\hline
\end{tabular}


Analysis 4.4. Comparison 4 Stratified analysis, by studies reporting 12 month data, Outcome 4 Steroidresistent rejection.

Review: Cyclosporin versus tacrolimus for liver transplanted patients
Comparison: 4 Stratified analysis, by studies reporting 12 month data
Outcome: 4 Steroid-resistent rejection

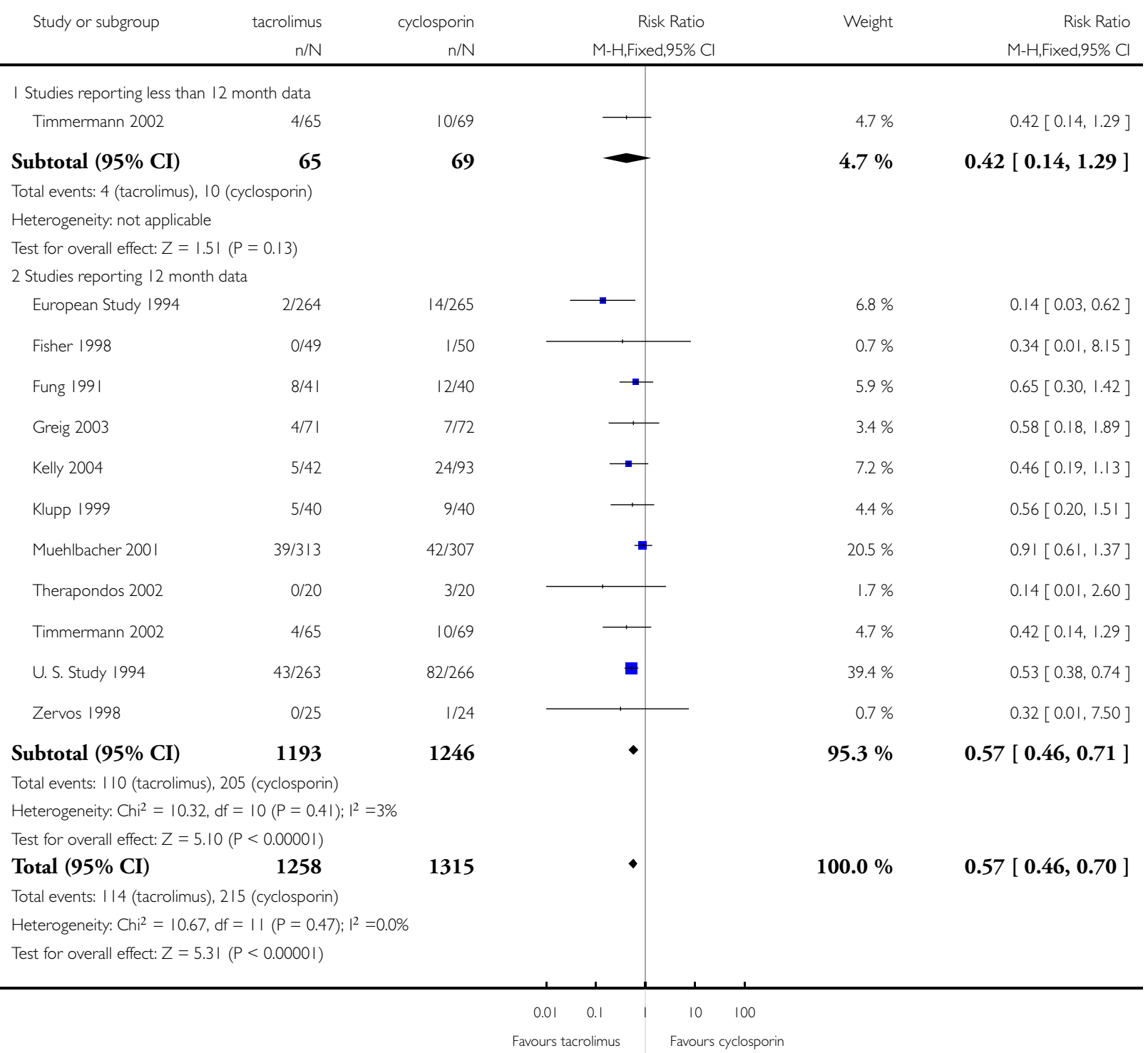


Analysis 4.6. Comparison 4 Stratified analysis, by studies reporting 12 month data, Outcome 6 Diabetes mellitus: initially diagnosed after transplantation.

Review: Cyclosporin versus tacrolimus for liver transplanted patients
Comparison: 4 Stratified analysis, by studies reporting 12 month data
Outcome: 6 Diabetes mellitus: initially diagnosed after transplantation

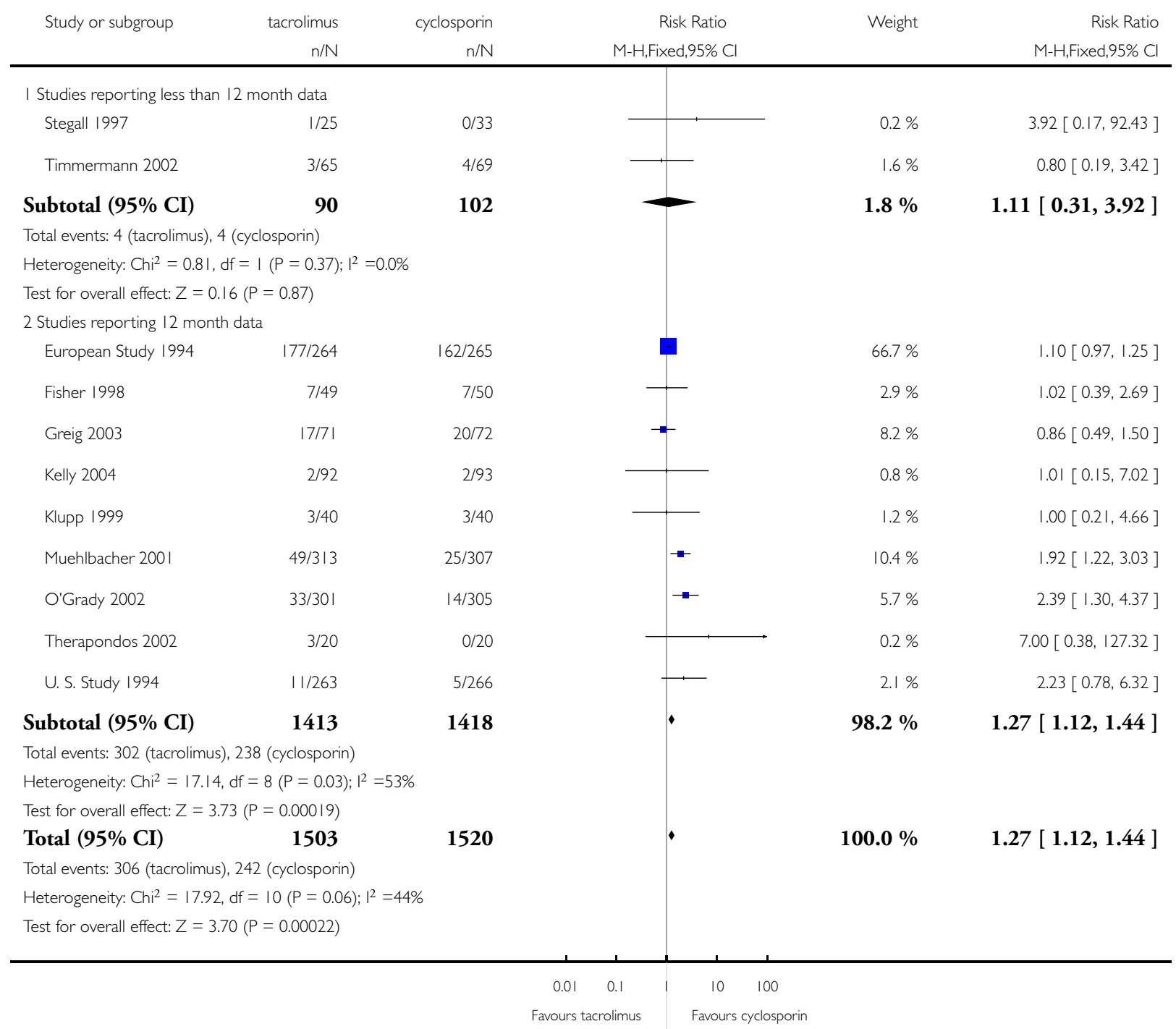


Analysis 4.8. Comparison 4 Stratified analysis, by studies reporting 12 month data, Outcome 8 Patients withdrawn from tacrolimus or cyclosporin.

Review: Cyclosporin versus tacrolimus for liver transplanted patients

Comparison: 4 Stratified analysis, by studies reporting 12 month data

Outcome: 8 Patients withdrawn from tacrolimus or cyclosporin

\begin{tabular}{|c|c|c|c|c|c|}
\hline \multirow[t]{2}{*}{ Study or subgroup } & tacrolimus & cyclosporin & \multirow{2}{*}{$\begin{array}{c}\text { Risk Ratio } \\
\text { M-H,Fixed,95\% Cl }\end{array}$} & \multirow[t]{2}{*}{ Weight } & \multirow{2}{*}{$\begin{array}{r}\text { Risk Ratio } \\
\text { M-H,Fixed,95\% Cl }\end{array}$} \\
\hline & $\mathrm{n} / \mathrm{N}$ & $\mathrm{n} / \mathrm{N}$ & & & \\
\hline \multicolumn{6}{|c|}{ I Studies reporting less than 12 month data } \\
\hline Stegall 1997 & $7 / 25$ & $6 / 32$ & 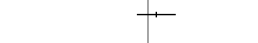 & $1.3 \%$ & $1.49[0.57,3.89]$ \\
\hline Subtotal (95\% CI) & 25 & 32 & & $1.3 \%$ & $1.49[0.57,3.89]$ \\
\hline \multicolumn{6}{|c|}{ Total events: 7 (tacrolimus), 6 (cyclosporin) } \\
\hline \multicolumn{6}{|c|}{ Heterogeneity: not applicable } \\
\hline \multicolumn{6}{|c|}{ Test for overall effect: $Z=0.82(P=0.4 \mathrm{I})$} \\
\hline \multicolumn{6}{|c|}{2 Studies reporting 12 month data } \\
\hline European Study 1994 & $76 / 264$ & $64 / 265$ & 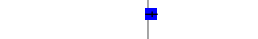 & $16.3 \%$ & $1.19[0.90,1.59]$ \\
\hline Fisher 1998 & $7 / 48$ & $10 / 49$ & $\rightarrow$ & $2.5 \%$ & $0.71[0.30,1.72]$ \\
\hline Fung 1991 & $1 / 79$ & $47 / 75$ & $\longleftrightarrow$ & $12.3 \%$ & $0.02[0.00,0.14]$ \\
\hline Greig 2003 & $7 / 71$ & $7 / 72$ & + & $1.8 \%$ & $1.01[0.37,2.74]$ \\
\hline Kelly 2004 & $2|/ 9|$ & $43 / 90$ & - & $11.0 \%$ & $0.48[0.31,0.74]$ \\
\hline Klupp 1999 & $5 / 40$ & $19 / 40$ & + & $4.8 \%$ & $0.26[0.11,0.64]$ \\
\hline Martin 2004 & $6 / 38$ & $4 / 41$ & + & $1.0 \%$ & $1.62[0.49,5.30]$ \\
\hline Muehlbacher 2001 & $4 / 310$ & $18 / 305$ & $\longrightarrow$ & $4.6 \%$ & $0.22[0.07,0.64]$ \\
\hline O'Grady 2002 & $33 / 301$ & $57 / 305$ & $*$ & $14.4 \%$ & $0.59[0.39,0.87]$ \\
\hline Therapondos 2002 & $2 / 18$ & $4 / 19$ & -1 & $1.0 \%$ & $0.53[0.11,2.54]$ \\
\hline U. S. Study 1994 & $83 / 263$ & $102 / 266$ & $\mathbf{1}$ & $25.9 \%$ & $0.82[0.65,1.04]$ \\
\hline Zervos 1998 & $0 / 25$ & $11 / 24$ & - & $3.0 \%$ & $0.04[0.00,0.67]$ \\
\hline Subtotal (95\% CI) & 1548 & 1551 & 4 & $98.7 \%$ & $0.64[0.55,0.73]$ \\
\hline \multicolumn{6}{|c|}{ Total events: 245 (tacrolimus), 386 (cyclosporin) } \\
\hline \multicolumn{6}{|c|}{ Heterogeneity: Chi $^{2}=51.47, \mathrm{df}=1 \mathrm{I}(\mathrm{P}<0.0000 \mathrm{I}) ; \mathrm{I}^{2}=79 \%$} \\
\hline \multicolumn{6}{|c|}{ Test for overall effect: $Z=6.31(P<0.0000 \mathrm{I})$} \\
\hline Total (95\% CI) & 1573 & 1583 & $\cdot$ & $100.0 \%$ & $0.65[0.57,0.74]$ \\
\hline \multicolumn{6}{|c|}{ Total events: 252 (tacrolimus), 392 (cyclosporin) } \\
\hline \multicolumn{6}{|c|}{ Heterogeneity: $\mathrm{Ch}^{2}=53.32, \mathrm{df}=12(\mathrm{P}<0.0000 \mathrm{I}) ; \mathbf{1}^{2}=77 \%$} \\
\hline Test for overall effect: $Z=$ & $<0.00001)$ & & & & \\
\hline
\end{tabular}

$0.0010 .01 \quad 0.1 \quad 1 \quad 10 \quad 100 \quad 1000$

Favours tacrolimus Favours cyclosporin 
Analysis 5.I. Comparison 5 Stratified analysis, by studies confined to patients with hepatitis C virus, Outcome I Mortality.

Review: Cyclosporin versus tacrolimus for liver transplanted patients

Comparison: 5 Stratified analysis, by studies confined to patients with hepatitis $C$ virus

Outcome: I Mortality

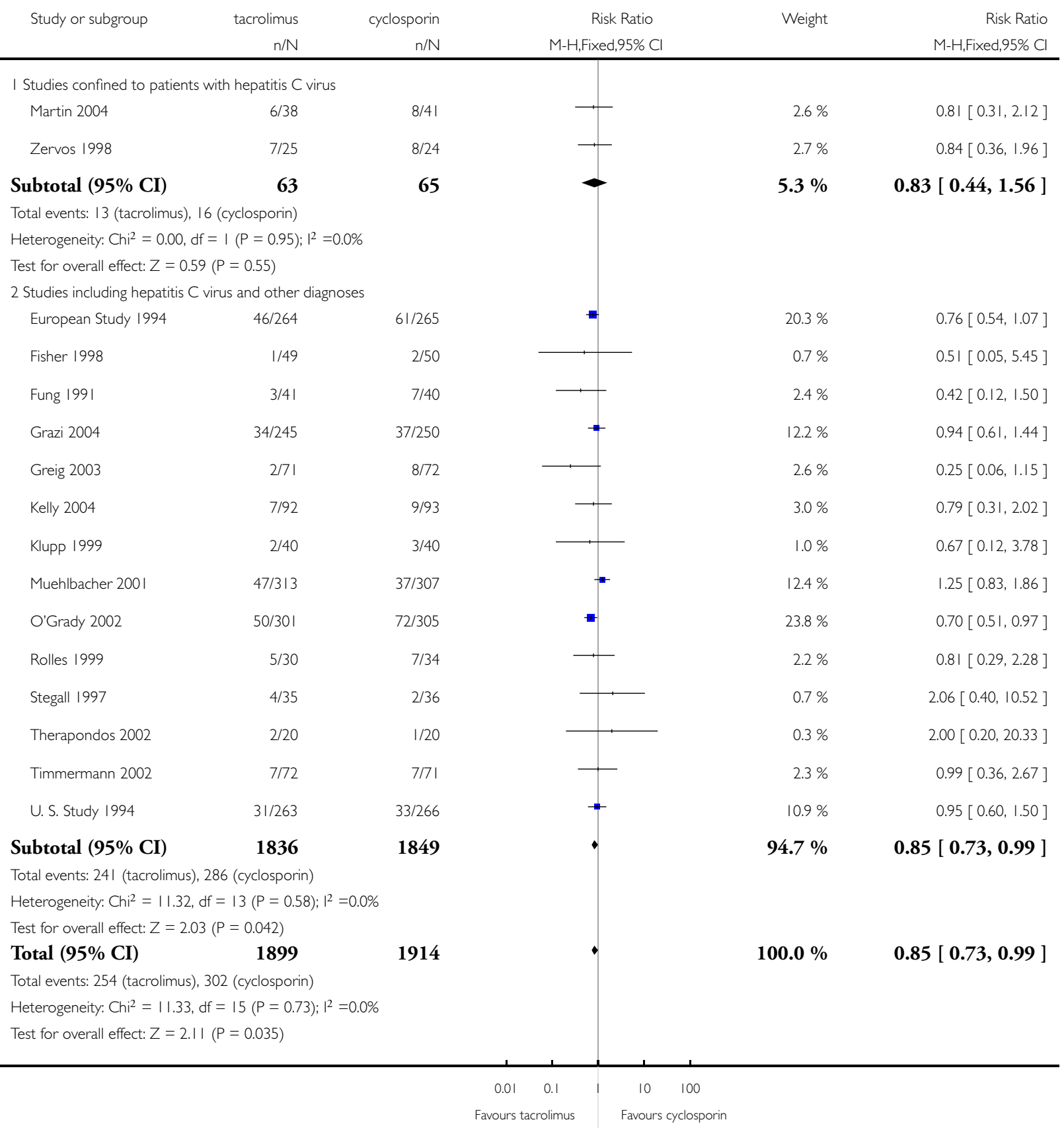


Analysis 5.2. Comparison $\mathbf{5}$ Stratified analysis, by studies confined to patients with hepatitis $\mathbf{C}$ virus, Outcome 2 Graft loss.

Review: Cyclosporin versus tacrolimus for liver transplanted patients

Comparison: 5 Stratified analysis, by studies confined to patients with hepatitis $C$ virus

Outcome: 2 Graft loss

$\begin{array}{llll}\text { Study or subgroup } & \text { tacrolimus } & \text { cyclosporin } & \text { Reight Ratio }\end{array}$

$\mathrm{n} / \mathrm{N}$

$n / N$

M-H,Fixed,95\% Cl

M-H,Fixed,95\% Cl

I Studies confined to patients with hepatitis $C$ virus

Martin 2004

$7 / 38$

$10 / 41$

Zervos 1998

$8 / 25$

$12 / 24$

Subtotal (95\% CI)

63

65

Total events: 15 (tacrolimus), 22 (cyclosporin)

Heterogeneity: $\mathrm{Chi}^{2}=0.09, \mathrm{df}=\mathrm{I}(\mathrm{P}=0.77) ; \mathrm{I}^{2}=0.0 \%$

Test for overall effect: $Z=1.33(P=0.18)$

2 Studies including hepatitis $C$ virus and other diagnoses

$\begin{array}{lrr}\text { European Study } 1994 & 60 / 264 & 74 / 265 \\ \text { Fisher 1998 } & 3 / 49 & 5 / 50 \\ \text { Fung 199| } & 4 / 41 & 12 / 40 \\ \text { Grazi 2004 } & 29 / 245 & 27 / 250 \\ \text { Greig 2003 } & 2 / 71 & 10 / 72 \\ \text { Kelly 2004 } & 7 / 92 & 13 / 93 \\ \text { Klupp 1999 } & 0 / 40 & 4 / 40 \\ \text { Muehlbacher 200। } & 53 / 313 & 52 / 307 \\ \text { O'Grady 2002 } & 58 / 301 & 88 / 305 \\ \text { Rolles 1999 } & 8 / 30 & 13 / 34 \\ \text { Stegall 1997 } & 5 / 35 & 3 / 36 \\ \text { Therapondos 2002 } & 2 / 20 & 1 / 20 \\ \text { Timmermann 2002 } & 10 / 72 & 13 / 7 \text { | } \\ \text { U. S. Study 1994 } & 48 / 263 & 55 / 266\end{array}$

Subtotal (95\% CI)

1836

1849

Total events: 289 (tacrolimus), 370 (cyclosporin)

Heterogeneity: $\mathrm{Chi}^{2}=15.77, \mathrm{df}=13(\mathrm{P}=0.26) ; \mathrm{I}^{2}=18 \%$

Test for overall effect: $Z=3.39(P=0.00070)$

Total (95\% CI)

1899

1914

Total events: 304 (tacrolimus), 392 (cyclosporin)

Heterogeneity: Chi $^{2}=16.15, \mathrm{df}=15(P=0.37) ;\left.\right|^{2}=7 \%$

Test for overall effect: $Z=3.59(P=0.00033)$

$0.81[0.61,1.09]$

$0.61[0.15,2.42]$

$0.33[0.11,0.92]$

।.10[0.67, 1.80$]$

$0.20[0.05,0.89]$

$0.54[0.23,1.30]$

$0.11[0.01,2.00]$

$1.00[0.71,1.42]$

$0.67[0.50,0.89]$

$0.70[0.34,1.45]$

I.7। $[0.44,6.64]$

$2.00[0.20,20.33]$

$0.76[0.36,1.62]$

$0.88[0.62,1.25]$

$94.4 \%$

$0.79[0.69,0.90]$

$100.0 \%$

$0.78[0.68,0.89]$

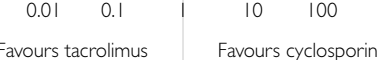


Analysis 5.3. Comparison $\mathbf{5}$ Stratified analysis, by studies confined to patients with hepatitis $\mathbf{C}$ virus, Outcome 3 Acute rejection.

Review: Cyclosporin versus tacrolimus for liver transplanted patients

Comparison: 5 Stratified analysis, by studies confined to patients with hepatitis $C$ virus

Outcome: 3 Acute rejection

\begin{tabular}{|c|c|c|c|c|c|}
\hline \multirow[t]{2}{*}{ Study or subgroup } & tacrolimusTreatment & cyclosporin & \multirow{2}{*}{$\begin{array}{c}\text { Risk Ratio } \\
\text { M-H,Fixed,95\% Cl }\end{array}$} & \multirow[t]{2}{*}{ Weight } & \multirow{2}{*}{$\begin{array}{r}\text { Risk Ratio } \\
\text { M-H,Fixed,95\% Cl }\end{array}$} \\
\hline & $\mathrm{n} / \mathrm{N}$ & $\mathrm{n} / \mathrm{N}$ & & & \\
\hline \multicolumn{6}{|c|}{ I Studies confined to patients with hepatitis $C$ virus } \\
\hline Martin 2004 & $11 / 38$ & $|6 / 4|$ & $\longrightarrow$ & $1.7 \%$ & $0.74[0.40,1.39]$ \\
\hline Zervos 1998 & $6 / 25$ & $12 / 24$ & 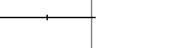 & $1.4 \%$ & $0.48[0.21,1.07]$ \\
\hline Subtotal (95\% CI) & 63 & 65 & & $3.1 \%$ & $0.63[0.38,1.02]$ \\
\hline \multicolumn{6}{|c|}{ Total events: 17 (tacrolimusTreatment), 28 (cyclosporin) } \\
\hline \multicolumn{6}{|c|}{ Heterogeneity: $\mathrm{Chi}^{2}=0.70, \mathrm{df}=\mathrm{I}(\mathrm{P}=0.40) ; \mathrm{I}^{2}=0.0 \%$} \\
\hline \multicolumn{6}{|c|}{ Test for overall effect: $Z=1.87(P=0.062)$} \\
\hline \multicolumn{6}{|c|}{2 Studies including hepatitis $C$ virus and other diagnoses } \\
\hline European Study 1994 & $115 / 264$ & $142 / 265$ & \# & $16.0 \%$ & $0.81[0.68,0.97]$ \\
\hline Fisher 1998 & $5 / 49$ & $2 / 50$ & 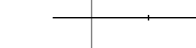 & $0.2 \%$ & $2.55[0.52,12.53]$ \\
\hline Fung 1991 & $19 / 4 \mid$ & $29 / 33$ & 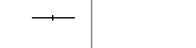 & $3.6 \%$ & $0.53[0.37,0.75]$ \\
\hline Grazi 2004 & $12 / 245$ & $18 / 250$ & $\longrightarrow$ & $2.0 \%$ & $0.68[0.33,1.38]$ \\
\hline Greig 2003 & $25 / 7 \mid$ & $31 / 72$ & $\div$ & $3.5 \%$ & $0.82[0.54,1.24]$ \\
\hline Kelly 2004 & $38 / 92$ & $49 / 93$ & $\rightarrow$ & $5.5 \%$ & $0.78[0.57,1.07]$ \\
\hline Klupp 1999 & $18 / 40$ & $30 / 40$ & $\longrightarrow$ & $3.4 \%$ & $0.60[0.41,0.88]$ \\
\hline Muehlbacher 2001 & $119 / 313$ & |35/307 & - & $15.4 \%$ & $0.86[0.72,1.05]$ \\
\hline O'Grady 2002 & $|43 / 30|$ & $179 / 305$ & $=$ & $20.1 \%$ & $0.81[0.70,0.94]$ \\
\hline Rolles 1999 & $20 / 30$ & $22 / 34$ & 7 & $2.3 \%$ & $1.03[0.72,1.47]$ \\
\hline Stegall 1997 & $11 / 26$ & $15 / 32$ & 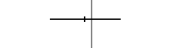 & $1.5 \%$ & $0.90[0.50,1.61]$ \\
\hline Therapondos 2002 & $8 / 20$ & $3 / 20$ & & $0.3 \%$ & $2.67[0.82,8.62]$ \\
\hline Timmermann 2002 & $24 / 65$ & $32 / 69$ & + & $3.5 \%$ & $0.80[0.53,1.20]$ \\
\hline U. S. Study 1994 & $154 / 265$ & $173 / 266$ & 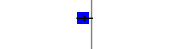 & $19.5 \%$ & $0.89[0.78,1.02]$ \\
\hline Subtotal (95\% CI) & 1822 & 1836 & - & $96.9 \%$ & $0.83[0.77,0.89]$ \\
\hline \multicolumn{6}{|c|}{ Total events: 7II (tacrolimusTreatment), 860 (cyclosporin) } \\
\hline \multicolumn{6}{|c|}{ Heterogeneity: $\mathrm{Chi}^{2}=18.24, \mathrm{df}=13(\mathrm{P}=0.15) ; \mathrm{I}^{2}=29 \%$} \\
\hline \multicolumn{6}{|c|}{ Test for overall effect: $Z=5.16(P<0.0000 I)$} \\
\hline Total (95\% CI) & 1885 & 1901 & - & $100.0 \%$ & $0.82[0.77,0.88]$ \\
\hline \multicolumn{6}{|c|}{ Total events: 728 (tacrolimusTreatment), 888 (cyclosporin) } \\
\hline \multicolumn{6}{|c|}{ Heterogeneity: $\mathrm{Chi}^{2}=20.10, \mathrm{df}=15(P=0.17) ;\left.\right|^{2}=25 \%$} \\
\hline Test for overall effect: $Z=$ & $(P<0.0000 I)$ & & & & \\
\hline
\end{tabular}


Analysis 5.4. Comparison 5 Stratified analysis, by studies confined to patients with hepatitis $\mathbf{C}$ virus, Outcome 4 Steroid-resistent rejection.

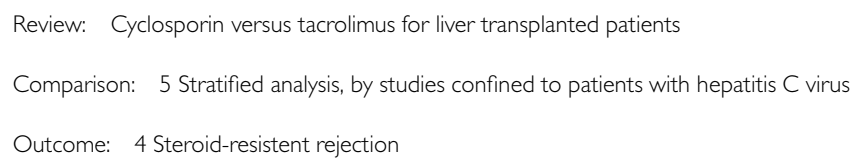

$\mathrm{n} / \mathrm{N} \quad \mathrm{n} / \mathrm{N} \quad \mathrm{M}-\mathrm{H}$, Fixed,95\% Cl M-H,Fixed,95\% Cl

I Studies confined to patients with hepatitis $C$ virus

Zervos 1998

Subtotal (95\% CI)

Total events: 0 (tacrolimus), I (cyclosporin)

Heterogeneity: not applicable

Test for overall effect: $Z=0.7 \mid(P=0.48)$

2 Studies including hepatitis $C$ virus and other diagnoses

European Study $1994 \quad$ 2/264

Fisher 1998

Fung 1991

Total events: I 10 (tacrolimus), 204 (cyclosporin) Heterogeneity: Chi $^{2}=10.17, \mathrm{df}=9(\mathrm{P}=0.34) ; \mathrm{I}^{2}=11 \%$

Test for overall effect: $Z=5.06(P<0.0000 \mathrm{I})$

Total (95\% CI)

1193

Total events: I 10 (tacrolimus), 205 (cyclosporin) Heterogeneity: Chi $^{2}=10.32, \mathrm{df}=10(P=0.4 \mathrm{I}) ; \mathrm{I}^{2}=3 \%$ Test for overall effect: $Z=5.10(P<0.0000 I)$

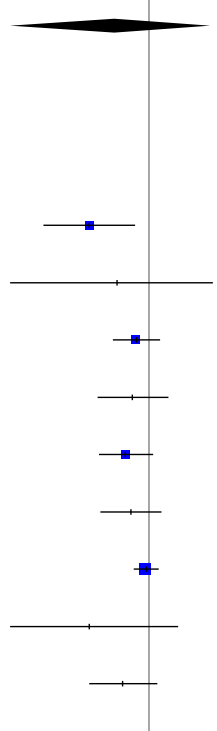


Analysis 5.5. Comparison 5 Stratified analysis, by studies confined to patients with hepatitis $\mathbf{C}$ virus, Outcome 5 Post transplant lymphoproliferative disease.

Review: Cyclosporin versus tacrolimus for liver transplanted patients

Comparison: 5 Stratified analysis, by studies confined to patients with hepatitis $C$ virus

Outcome: 5 Post transplant lymphoproliferative disease

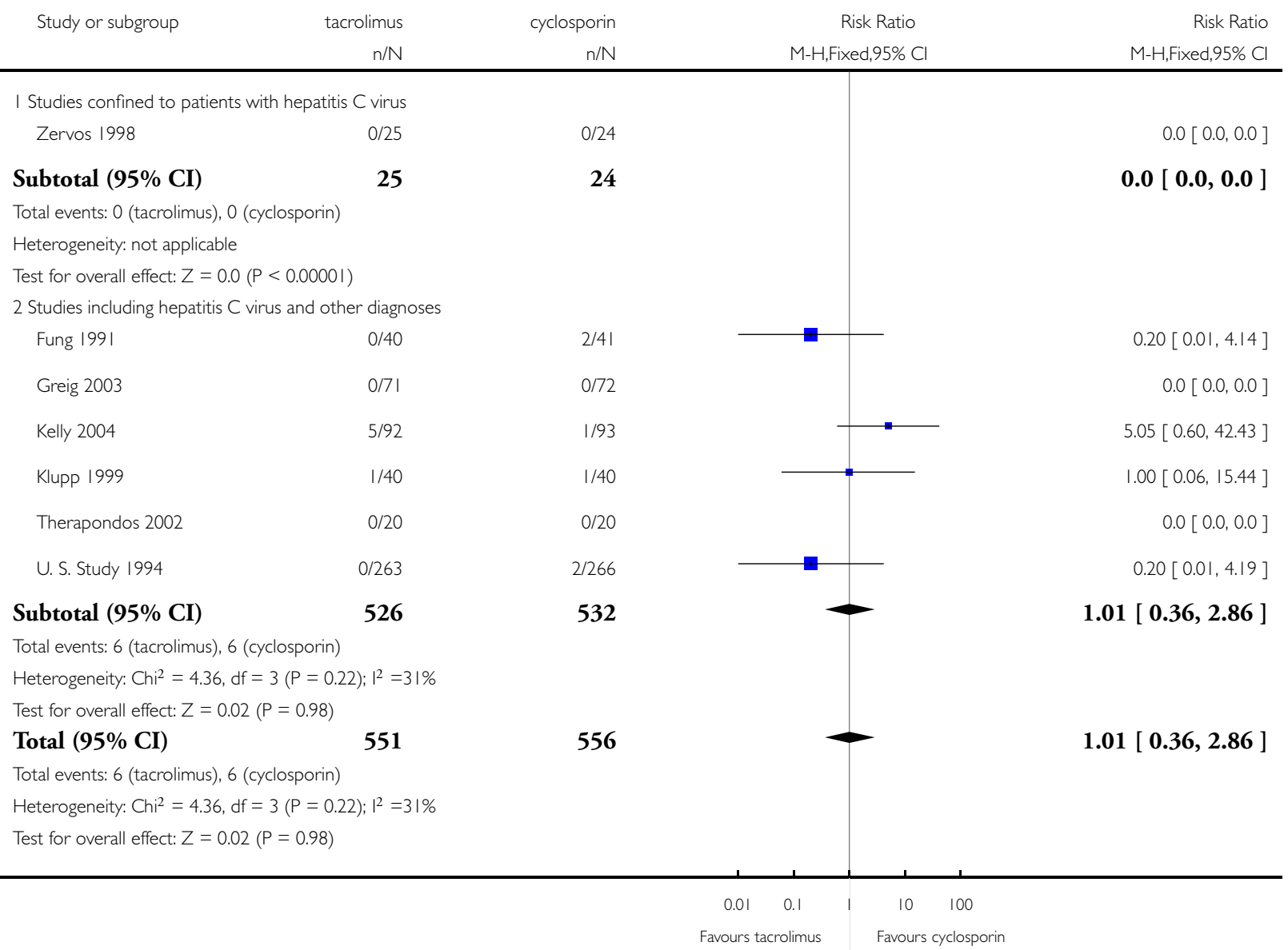


Analysis 5.6. Comparison 5 Stratified analysis, by studies confined to patients with hepatitis $\mathbf{C}$ virus, Outcome 6 Patients withdrawn from tacrolimus or cyclosporin.

Review: Cyclosporin versus tacrolimus for liver transplanted patients

Comparison: 5 Stratified analysis, by studies confined to patients with hepatitis $C$ virus

Outcome: 6 Patients withdrawn from tacrolimus or cyclosporin

\begin{tabular}{|c|c|c|c|c|c|}
\hline \multirow[t]{2}{*}{ Study or subgroup } & tacrolimus & cyclosporin & \multirow{2}{*}{$\begin{array}{c}\text { Risk Ratio } \\
\text { M-H,Fixed,95\% Cl }\end{array}$} & \multirow[t]{2}{*}{ Weight } & \multirow{2}{*}{$\begin{array}{r}\text { Risk Ratio } \\
\text { M-H,Fixed,95\% Cl }\end{array}$} \\
\hline & $\mathrm{n} / \mathrm{N}$ & $\mathrm{n} / \mathrm{N}$ & & & \\
\hline \multicolumn{6}{|c|}{ I Studies confined to patients with hepatitis $C$ virus } \\
\hline Martin 2004 & $6 / 38$ & $4 / 41$ & $\sqrt{1}$ & $1.0 \%$ & $1.62[0.49,5.30]$ \\
\hline Zervos 1998 & $0 / 25$ & $11 / 24$ & $\longleftarrow$ & $3.0 \%$ & $0.04[0.00,0.67]$ \\
\hline Subtotal (95\% CI) & 63 & 65 & 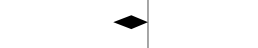 & $4.0 \%$ & $0.43[0.18,1.02]$ \\
\hline \multicolumn{6}{|c|}{ Total events: 6 (tacrolimus), 15 (cyclosporin) } \\
\hline \multicolumn{6}{|c|}{ Heterogeneity: $\mathrm{Chi}^{2}=7.49, \mathrm{df}=\mathrm{I}(\mathrm{P}=0.0 \mathrm{I}) ; \mathrm{I}^{2}=87 \%$} \\
\hline \multicolumn{6}{|c|}{ Test for overall effect: $Z=1.91(P=0.056)$} \\
\hline \multicolumn{6}{|c|}{2 Studies including hepatitis $C$ virus and other diagnoses } \\
\hline European Study 1994 & $76 / 264$ & $64 / 265$ & P & $16.3 \%$ & $1.19[0.90,1.59]$ \\
\hline Fisher 1998 & $7 / 48$ & $10 / 49$ & $\rightarrow$ & $2.5 \%$ & $0.71[0.30,1.72]$ \\
\hline Fung 1991 & $1 / 79$ & $47 / 75$ & $\longleftrightarrow$ & $12.3 \%$ & $0.02[0.00,0.14]$ \\
\hline Greig 2003 & $7 / 71$ & $7 / 72$ & + & $1.8 \%$ & $1.01[0.37,2.74]$ \\
\hline Kelly 2004 & $2|/ 9|$ & $43 / 90$ & $*$ & $11.0 \%$ & $0.48[0.31,0.74]$ \\
\hline Klupp 1999 & $5 / 40$ & $19 / 40$ & $\leftarrow$ & $4.8 \%$ & $0.26[0.11,0.64]$ \\
\hline Muehlbacher 2001 & $4 / 310$ & $18 / 305$ & $\longrightarrow$ & $4.6 \%$ & $0.22[0.07,0.64]$ \\
\hline O'Grady 2002 & $33 / 301$ & $57 / 305$ & - & $14.4 \%$ & $0.59[0.39,0.87]$ \\
\hline Stegall 1997 & $7 / 25$ & $6 / 32$ & 一 & $1.3 \%$ & $1.49[0.57,3.89]$ \\
\hline Therapondos 2002 & $2 / 18$ & $4 / 19$ & + & $1.0 \%$ & $0.53[0.11,2.54]$ \\
\hline U. S. Study 1994 & $83 / 263$ & $102 / 266$ & E & $25.9 \%$ & $0.82[0.65,1.04]$ \\
\hline Subtotal (95\% CI) & 1510 & 1518 & $\cdot$ & $96.0 \%$ & $0.66[0.57,0.76]$ \\
\hline \multicolumn{6}{|c|}{ Total events: 246 (tacrolimus), 377 (cyclosporin) } \\
\hline \multicolumn{6}{|c|}{ Heterogeneity: $\mathrm{Ch}^{2}=46.49, \mathrm{df}=10(\mathrm{P}<0.0000 \mathrm{I}) ; \mathrm{I}^{2}=78 \%$} \\
\hline \multicolumn{6}{|c|}{ Test for overall effect: $Z=5.87(P<0.0000 \mathrm{I})$} \\
\hline Total $(95 \% \mathrm{CI})$ & 1573 & 1583 & $\cdot$ & $100.0 \%$ & $0.65[0.57,0.74]$ \\
\hline \multicolumn{6}{|c|}{ Total events: 252 (tacrolimus), 392 (cyclosporin) } \\
\hline \multicolumn{6}{|c|}{ Heterogeneity: $\mathrm{Ch}^{2}=53.32, \mathrm{df}=12(\mathrm{P}<0.0000 \mathrm{I}) ; \mathrm{I}^{2}=77 \%$} \\
\hline Test for overall effect: $Z=$ & $<0.00001)$ & & & & \\
\hline
\end{tabular}

$0.0010 .01 \quad 0.1 \quad 1 \quad 10 \quad 100 \quad 1000$

Favours tacrolimus Favours cyclosporin 
Analysis 6.I. Comparison 6 Stratified analysis, by concomitant azathioprine or mycophenolate mofetil, Outcome I Mortality.

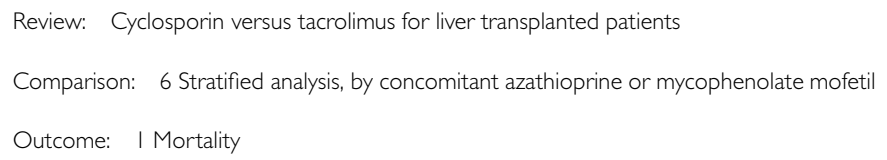

Total events: 34 (tacrolimus), 37 (cyclosporin)

Heterogeneity: not applicable

Test for overall effect: $Z=0.29(P=0.77)$

3 Concomitant azathioprine with cyclosporin only (some centres)

$\begin{array}{lrr}\text { European Study 1994 } & 46 / 264 & 61 / 265 \\ \text { Kelly 2004 } & 7 / 92 & 9 / 93 \\ \text { Muehlbacher 200 I } & 47 / 313 & 37 / 307 \\ \text { Timmermann 2002 } & 7 / 72 & 7 / 7 \\ \text { U. S. Study 1994 } & 31 / 263 & 33 / 266 \\ \text { ubtotal (95\% CI) } & \mathbf{1 0 0 4} & \mathbf{1 0 0 2}\end{array}$

Total events: 138 (tacrolimus), 147 (cyclosporin)

Heterogeneity: $\mathrm{Chi}^{2}=3.58, \mathrm{df}=4(\mathrm{P}=0.47) ; \mathrm{I}^{2}=0.0 \%$

Test for overall effect: $Z=0.59(P=0.55)$

4 Concomitant mycophenolate mofetil

Klupp 1999

Stegall 1997

$0.76[0.54,1.07]$

$0.79[0.31,2.02]$

$1.25[0.83,1.86]$

$0.99[0.36,2.67]$

$0.95[0.60,1.50]$
02

126

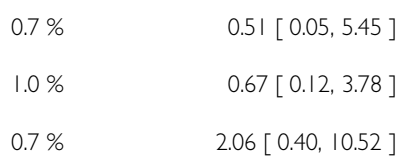

$2.3 \%$ 


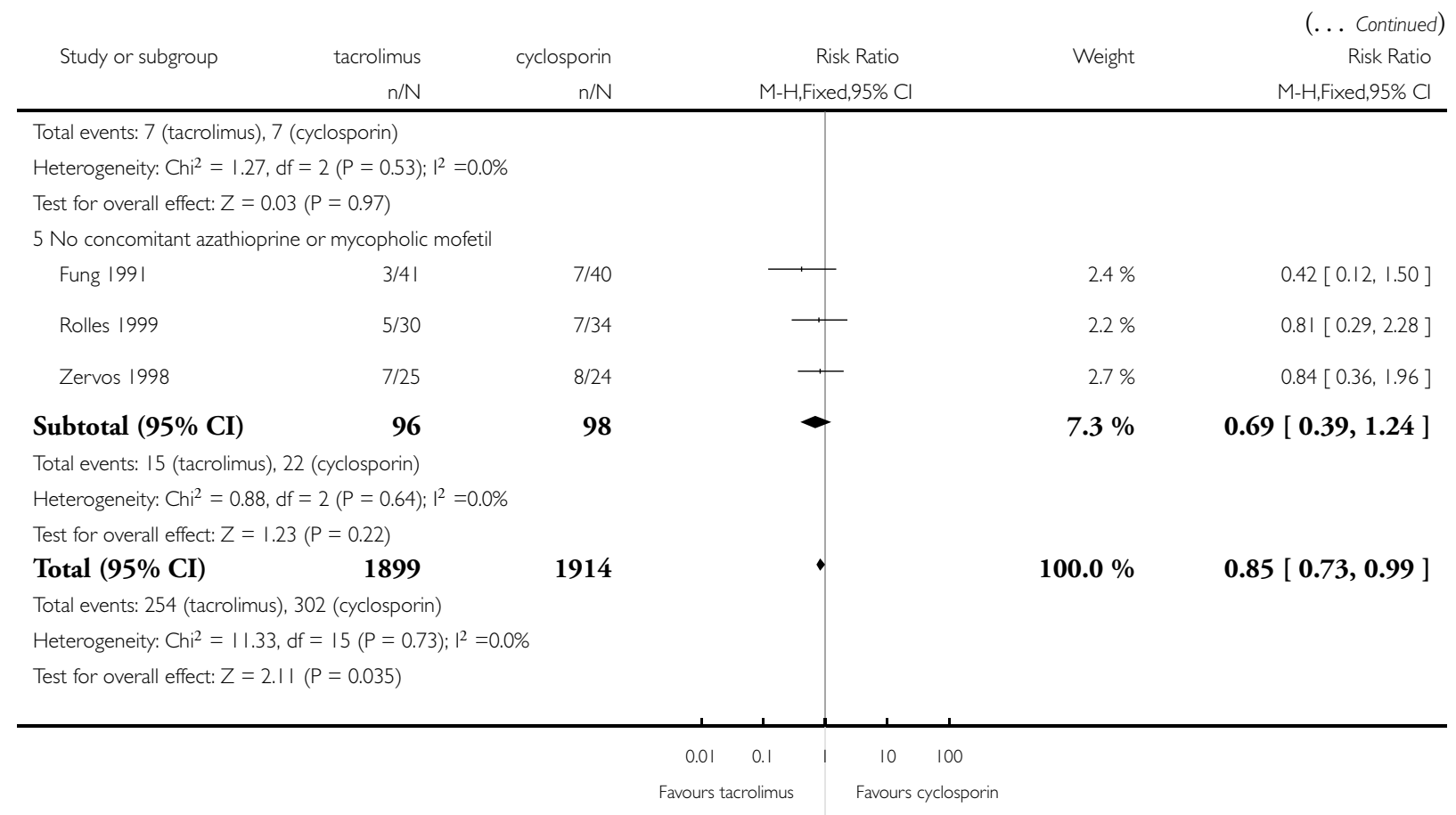


Analysis 6.2. Comparison 6 Stratified analysis, by concomitant azathioprine or mycophenolate mofetil, Outcome 2 Graft loss.

Review: Cyclosporin versus tacrolimus for liver transplanted patients

Comparison: 6 Stratified analysis, by concomitant azathioprine or mycophenolate mofetil

Outcome: 2 Graft loss

$\begin{array}{llll}\text { Study or subgroup } & \text { tacrolimus } & \text { cyclosporin } & \text { Risk Ratio }\end{array}$

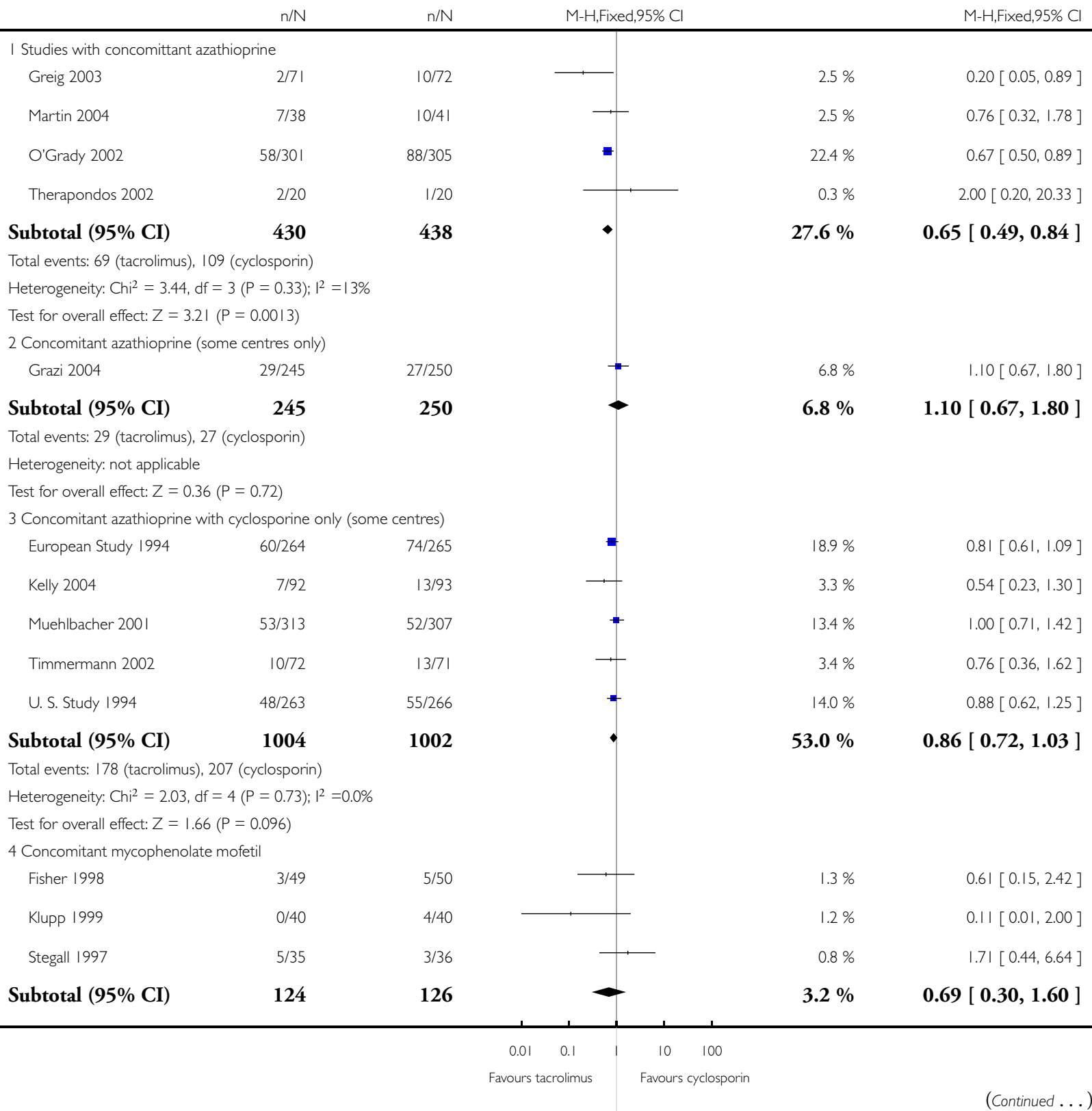




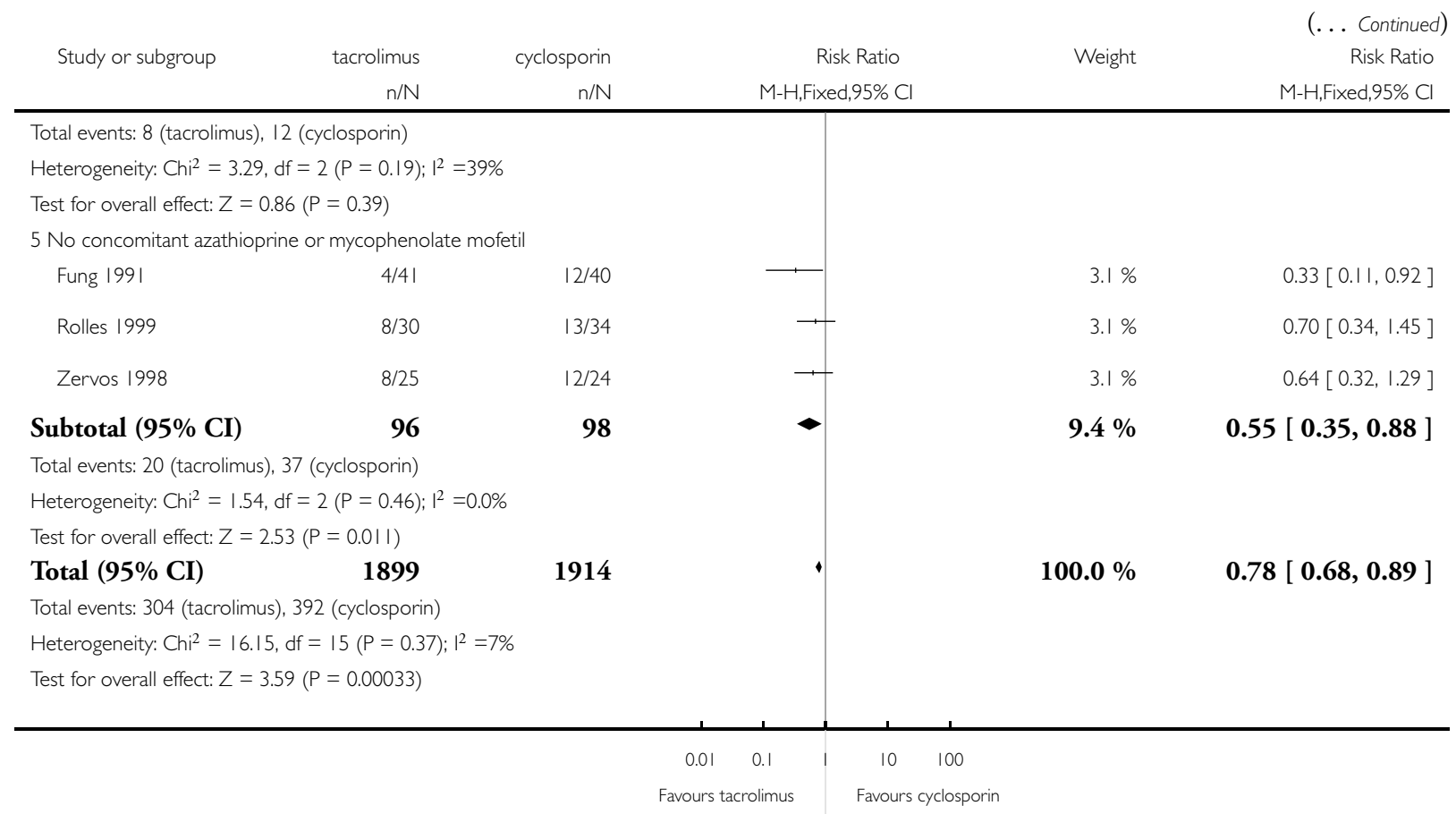


Analysis 6.3. Comparison 6 Stratified analysis, by concomitant azathioprine or mycophenolate mofetil, Outcome 3 Acute rejection.

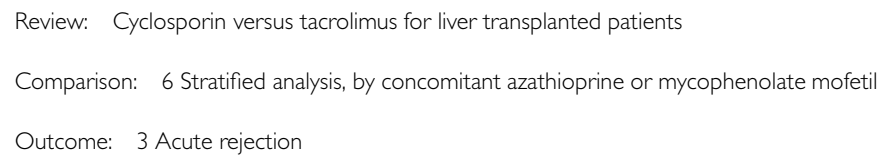

\begin{tabular}{lrr}
\hline $\begin{array}{l}\text { I Concomitant azathioprine } \\
\text { Greig } 2003\end{array}$ & $25 / 7 \mid$ & $31 / 72$ \\
Martin 2004 & $11 / 38$ & $16 / 4$ \\
O'Grady 2002 & $143 / 30 \mid$ & $179 / 305$ \\
Therapondos 2002 & $8 / 20$ & $3 / 2$
\end{tabular}

\section{Subtotal (95\% CI)}

430

Total events: 187 (tacrolimus), 229 (cyclosporin)

Test for overall effect: $Z=2.63(P=0.0087)$

2 Concomitant azathioprine (some centres only)

$$
\text { Grazi } 2004
$$

Subtotal (95\% CI)

Total events: 12 (tacrolimus), 18 (cyclosporin)

Heterogeneity: not applicable

Test for overall effect: $Z=1.07(P=0.29)$

3 Concomitant azathioprine with cyclosporin only (some centres)

$\begin{array}{lrr}\text { European Study 1994 } & \text { | | 5/264 } & \text { | } 42 / 265 \\ \text { Kelly 2004 } & 38 / 92 & 49 / 93 \\ \text { Muehlbacher 200 I } & 1 \text { | 19/3/3 } & 135 / 307 \\ \text { Timmermann 2002 } & 24 / 65 & 32 / 69 \\ \text { U. S. Study 1994 } & 154 / 265 & 173 / 266 \\ \text { Subtotal (95\% CI) } & \mathbf{9 9 9} & \mathbf{1 0 0 0}\end{array}$

\section{Subtotal (95\% CI)}

$$
999
$$$$
1000
$$

Total events: 450 (tacrolimus), 53। (cyclosporin)

Heterogeneity: $\mathrm{Chi}^{2}=1.17, \mathrm{df}=4(\mathrm{P}=0.88) ; \mathrm{I}^{2}=0.0 \%$

Test for overall effect: $Z=3.62(P=0.00029)$

4 Concomitant mycophenolate mofetil

$\begin{array}{lll}\text { Fisher } 1998 & 5 / 49 & 2 / 5 \\ \text { Klupp } 1999 & 18 / 40 & 30 / 40 \\ \text { Stegall } 1997 & 11 / 26 & 15 / 32\end{array}$

Subtotal (95\% CI)

$\begin{array}{ll}3.5 \% & 0.82[0.54,1.24] \\ 1.7 \% & 0.74[0.40,1.39] \\ 20.1 \% & 0.81[0.70,0.94] \\ 0.3 \% & 2.67[0.82,8.62]\end{array}$

$25.6 \%$

0.83 [ $0.72,0.95$ ] 


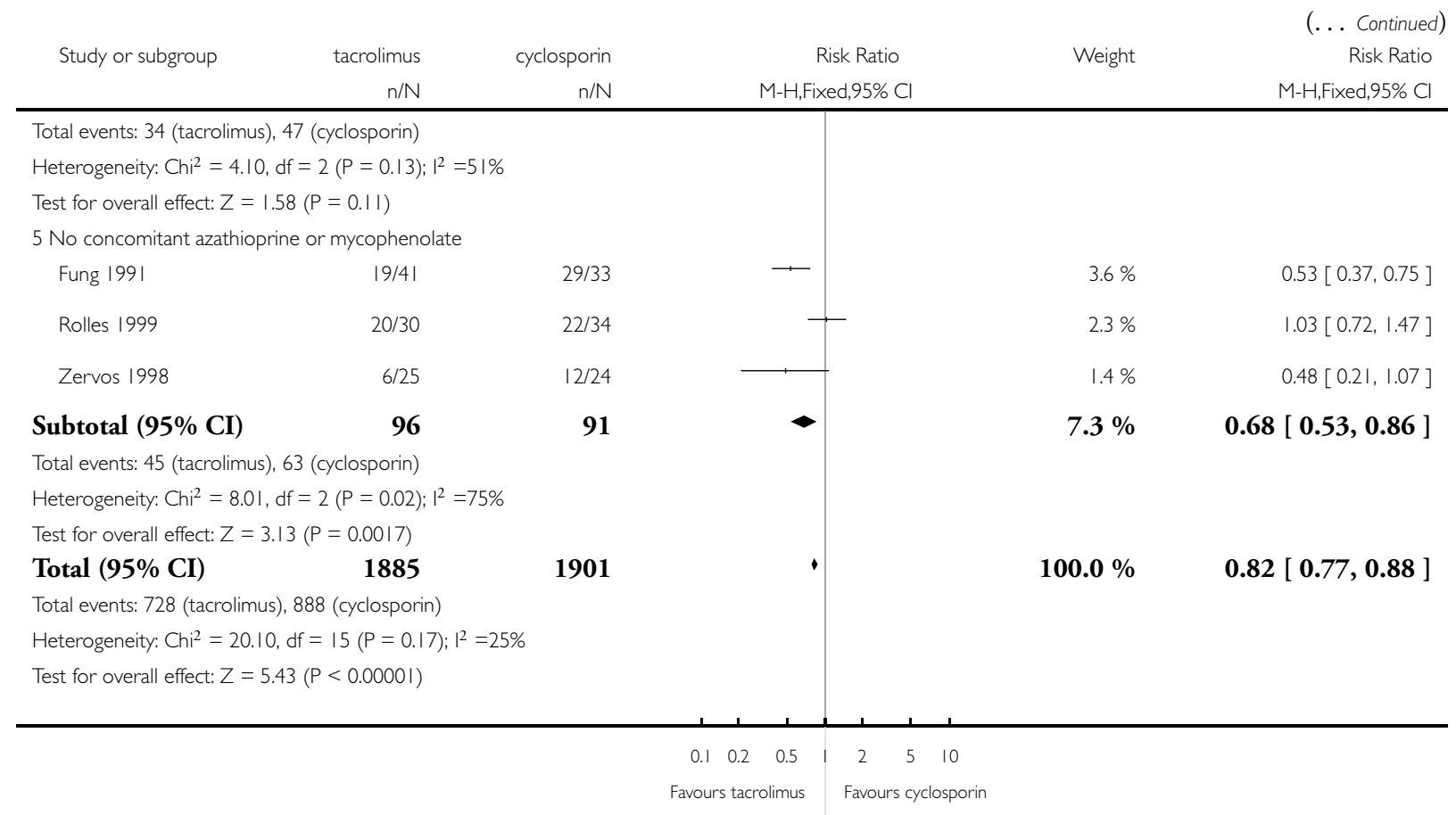


Analysis 6.4. Comparison 6 Stratified analysis, by concomitant azathioprine or mycophenolate mofetil, Outcome 4 Steroid-resistent rejection.

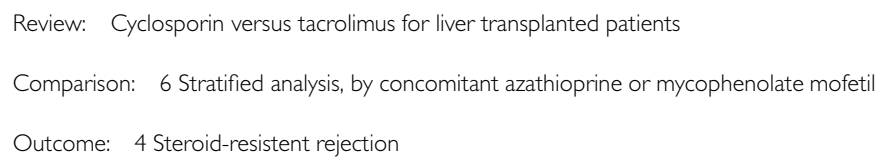

$\mathrm{n} / \mathrm{N} \quad \mathrm{n} / \mathrm{N} \quad \mathrm{M}-\mathrm{H}$, Fixed,95\% Cl $\quad \mathrm{M}-\mathrm{H}$, Fixed,95\% Cl

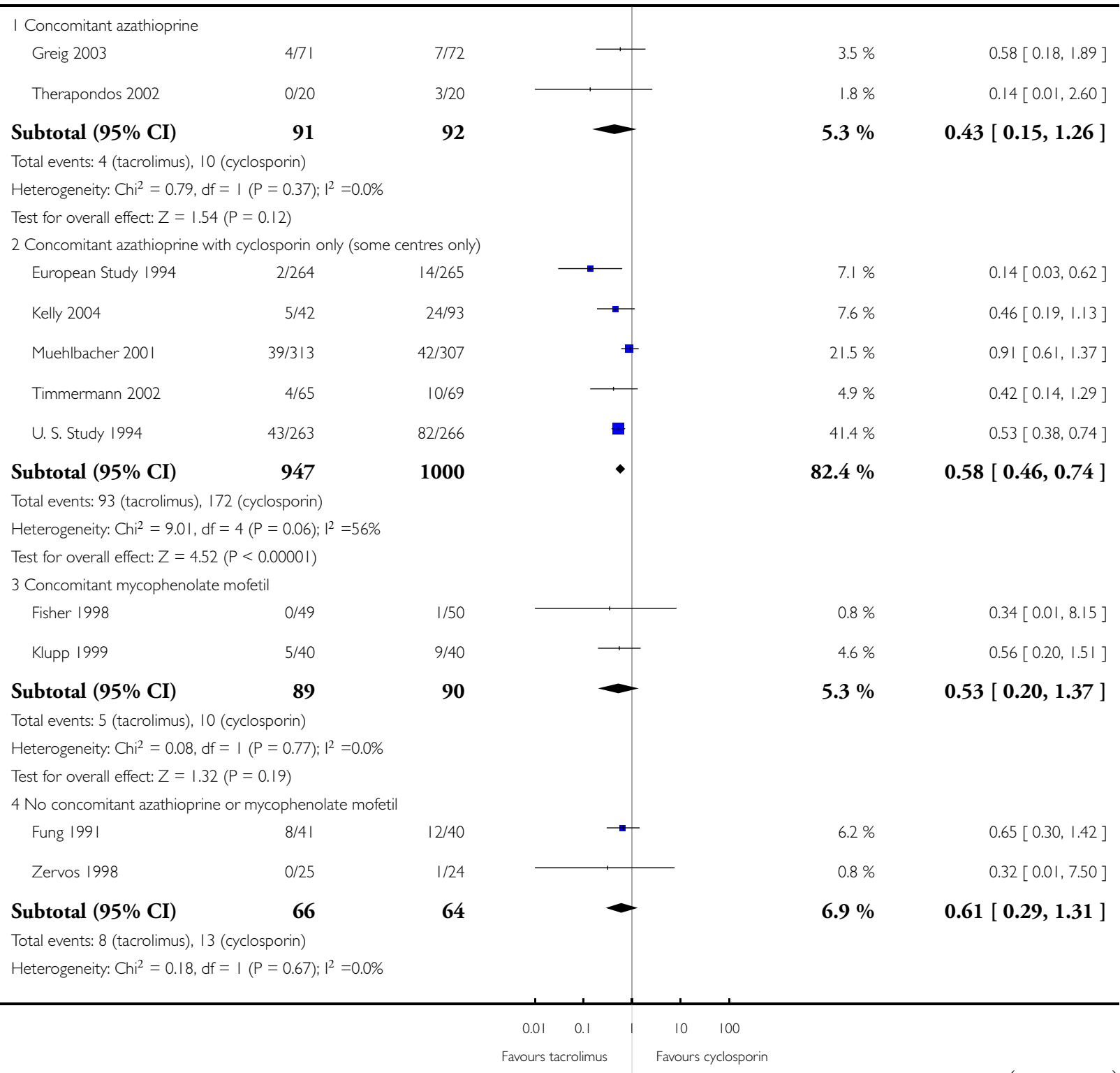




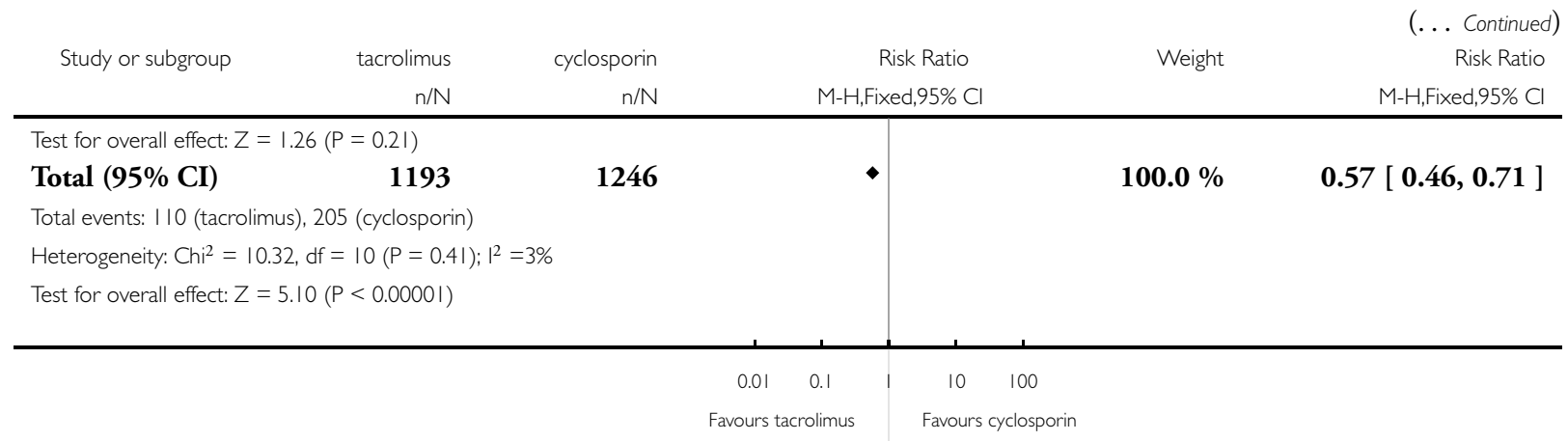

Analysis 6.5. Comparison 6 Stratified analysis, by concomitant azathioprine or mycophenolate mofetil, Outcome 5 Dialysis (de-novo requirement post-transplantation).

Review: Cyclosporin versus tacrolimus for liver transplanted patients

Comparison: 6 Stratified analysis, by concomitant azathioprine or mycophenolate mofetil

Outcome: 5 Dialysis (de-novo requirement post-transplantation)

$\begin{array}{llll}\text { Study or subgroup } & \text { tacrolimus } & \text { cyclosporin } & \text { Risk Ratio }\end{array}$

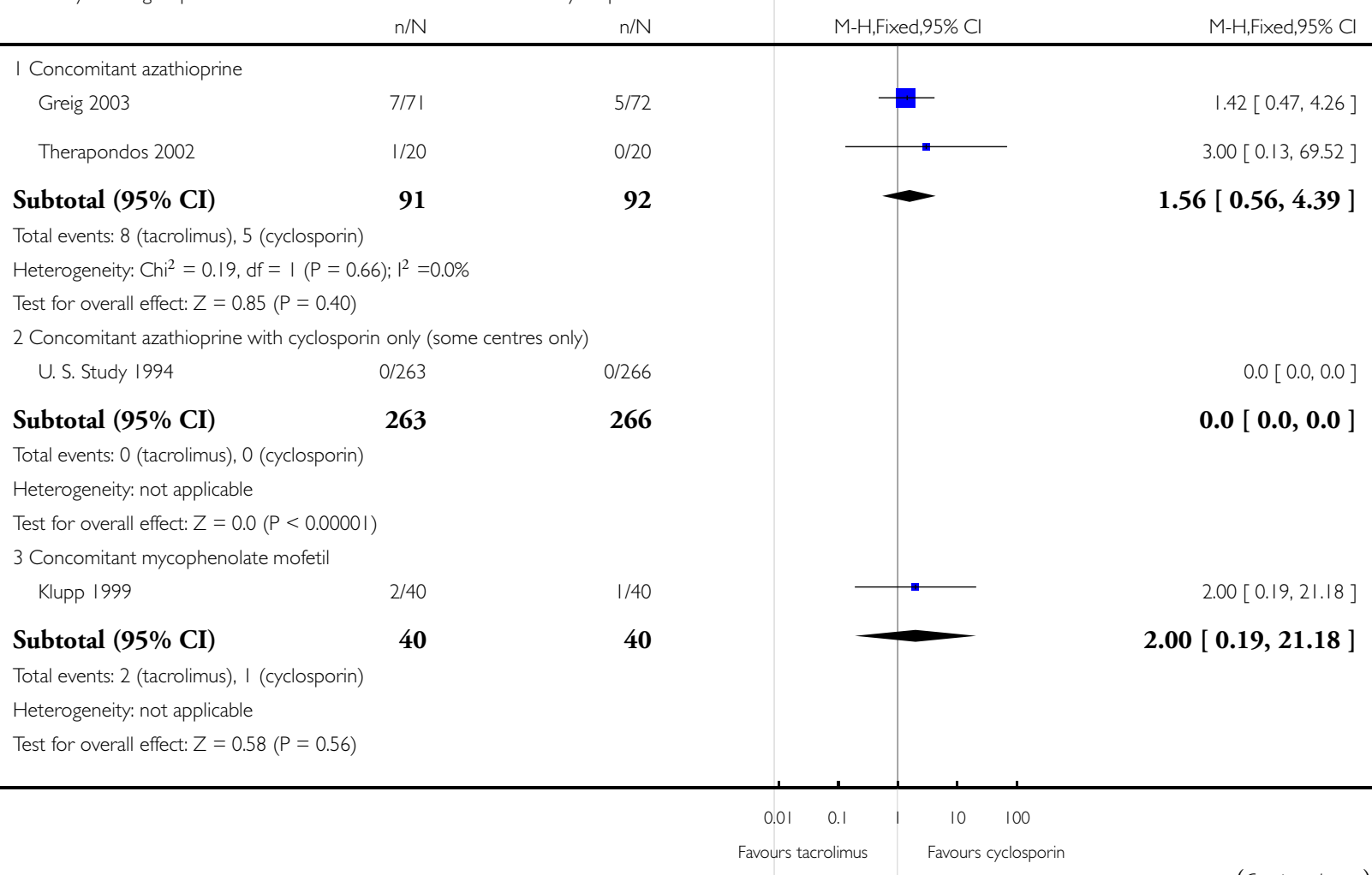

(Continued ... ) 


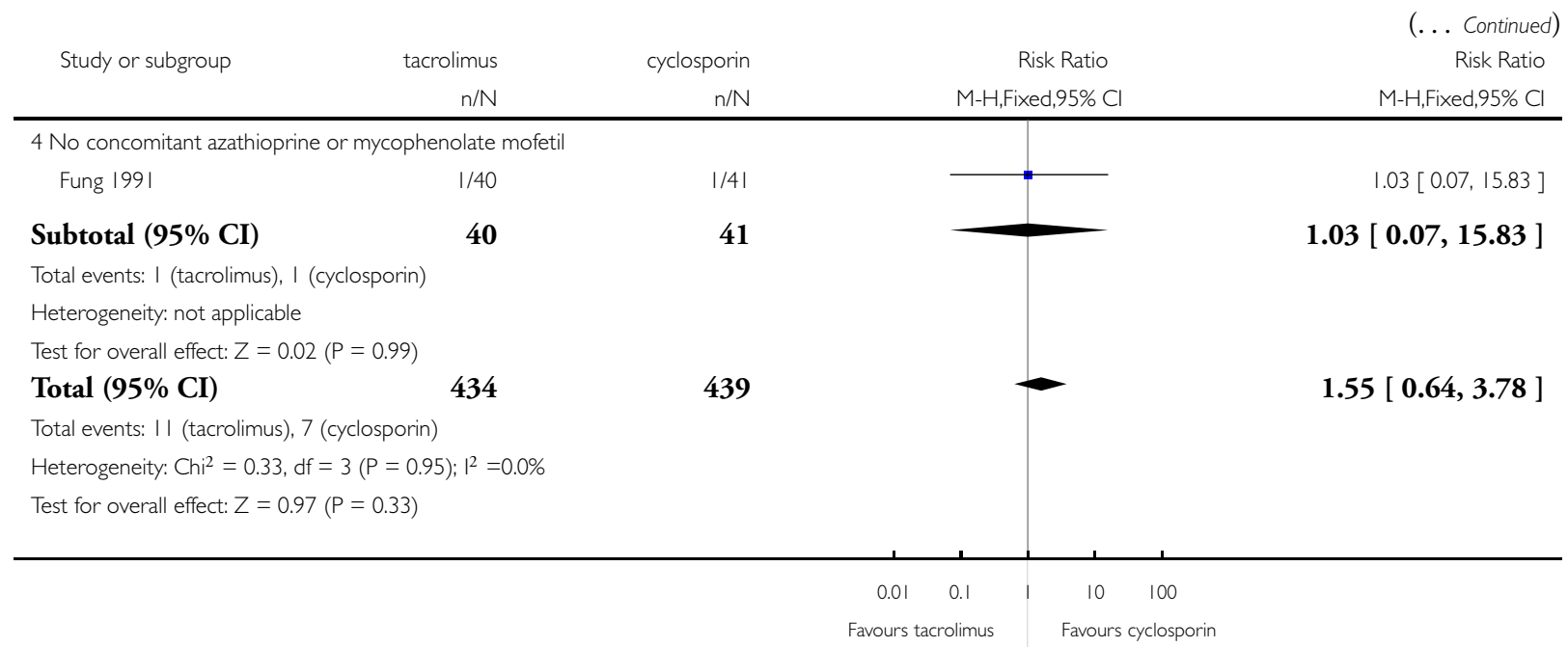

\section{Analysis 6.6. Comparison 6 Stratified analysis, by concomitant azathioprine or mycophenolate mofetil, Outcome 6 Diabetes mellitus: initially diagnosed after transplantation.}

Review: Cyclosporin versus tacrolimus for liver transplanted patients

Comparison: 6 Stratified analysis, by concomitant azathioprine or mycophenolate mofetil

Outcome: 6 Diabetes mellitus: initially diagnosed after transplantation

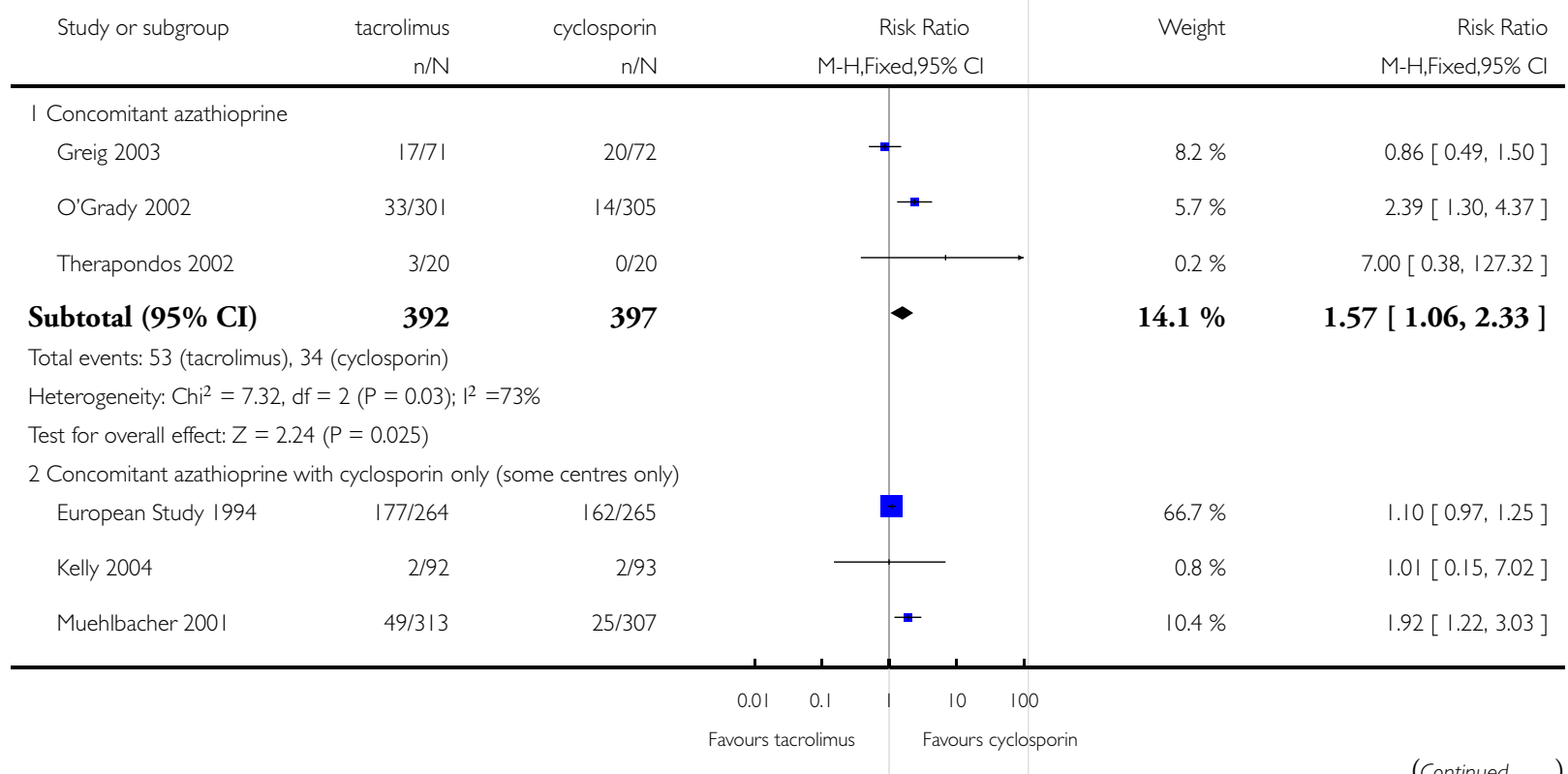




\begin{tabular}{|c|c|c|c|c|c|}
\hline \multirow[t]{2}{*}{ Study or subgroup } & tacrolimus & cyclosporin & Risk Ratio & \multirow[t]{2}{*}{ Weight } & \multirow{2}{*}{$\begin{array}{r}\text { (. . . Continued) } \\
\text { Risk Ratio } \\
\text { M-H,Fixed,95\% Cl }\end{array}$} \\
\hline & $n / N$ & $n / N$ & M-H,Fixed,95\% Cl & & \\
\hline Timmermann 2002 & $3 / 65$ & $4 / 69$ & $\longrightarrow$ & $1.6 \%$ & $0.80[0.19,3.42]$ \\
\hline U. S. Study 1994 & $11 / 263$ & $5 / 266$ & i & $2.1 \%$ & $2.23[0.78,6.32]$ \\
\hline Subtotal $(95 \% \mathrm{CI})$ & 997 & 1000 & - & $81.6 \%$ & $1.22[1.07,1.40]$ \\
\hline \multicolumn{6}{|c|}{ Total events: 242 (tacrolimus), 198 (cyclosporin) } \\
\hline \multicolumn{6}{|c|}{ Heterogeneity: $\mathrm{Chi}^{2}=8.23, \mathrm{df}=4(\mathrm{P}=0.08) ; \mathrm{I}^{2}=51 \%$} \\
\hline \multicolumn{6}{|c|}{ Test for overall effect: $Z=3.02(P=0.0026)$} \\
\hline \multicolumn{6}{|c|}{3 Concomitant mycophenolate mofetil } \\
\hline Fisher 1998 & $7 / 49$ & $7 / 50$ & + & $2.9 \%$ & $1.02[0.39,2.69]$ \\
\hline Klupp 1999 & $3 / 40$ & $3 / 40$ & 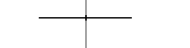 & $1.2 \%$ & $1.00[0.21,4.66]$ \\
\hline Stegall 1997 & 1/25 & 0/33 & 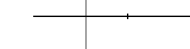 & $0.2 \%$ & $3.92[0.17,92.43]$ \\
\hline Subtotal $(95 \% \mathrm{CI})$ & 114 & 123 & 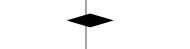 & $4.3 \%$ & $1.14[0.52,2.49]$ \\
\hline \multicolumn{6}{|c|}{ Total events: II (tacrolimus), I0 (cyclosporin) } \\
\hline \multicolumn{6}{|c|}{ Heterogeneity: $\mathrm{Ch}^{2} \mathrm{I}^{2}=0.66, \mathrm{df}=2(\mathrm{P}=0.72) ; \mathrm{I}^{2}=0.0 \%$} \\
\hline \multicolumn{6}{|c|}{ Test for overall effect: $Z=0.32(P=0.75)$} \\
\hline Total $(95 \% \mathrm{CI})$ & 1503 & 1520 & $\bullet$ & $100.0 \%$ & $1.27[1.12,1.44]$ \\
\hline \multicolumn{6}{|c|}{ Total events: 306 (tacrolimus), 242 (cyclosporin) } \\
\hline \multicolumn{6}{|c|}{ Heterogeneity: $\mathrm{Chi}^{2}=17.92, \mathrm{df}=10(\mathrm{P}=0.06) ; 1^{2}=44 \%$} \\
\hline Test for overall effect: $Z=$ & $=0.00022)$ & & & & \\
\hline
\end{tabular}


Analysis 6.7. Comparison 6 Stratified analysis, by concomitant azathioprine or mycophenolate mofetil, Outcome 7 Post transplant lymphoproliferative disease.

Review: Cyclosporin versus tacrolimus for liver transplanted patients

Comparison: 6 Stratified analysis, by concomitant azathioprine or mycophenolate mofetil

Outcome: 7 Post transplant lymphoproliferative disease

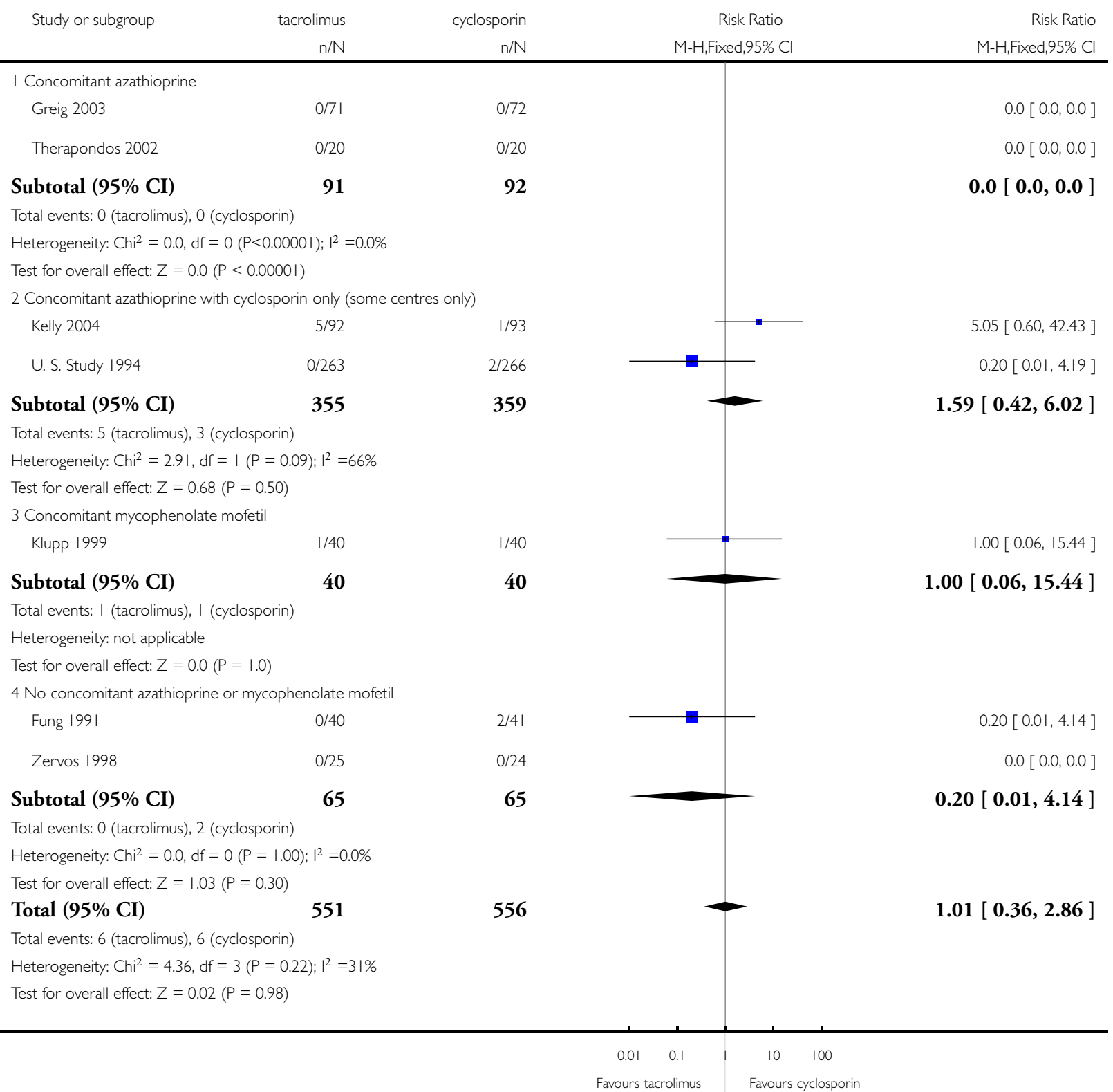


Analysis 6.8. Comparison 6 Stratified analysis, by concomitant azathioprine or mycophenolate mofetil, Outcome 8 Patients withdrawn from tacrolimus or cyclosporin.

Review: Cyclosporin versus tacrolimus for liver transplanted patients

Comparison: 6 Stratified analysis, by concomitant azathioprine or mycophenolate mofetil

Outcome: 8 Patients withdrawn from tacrolimus or cyclosporin

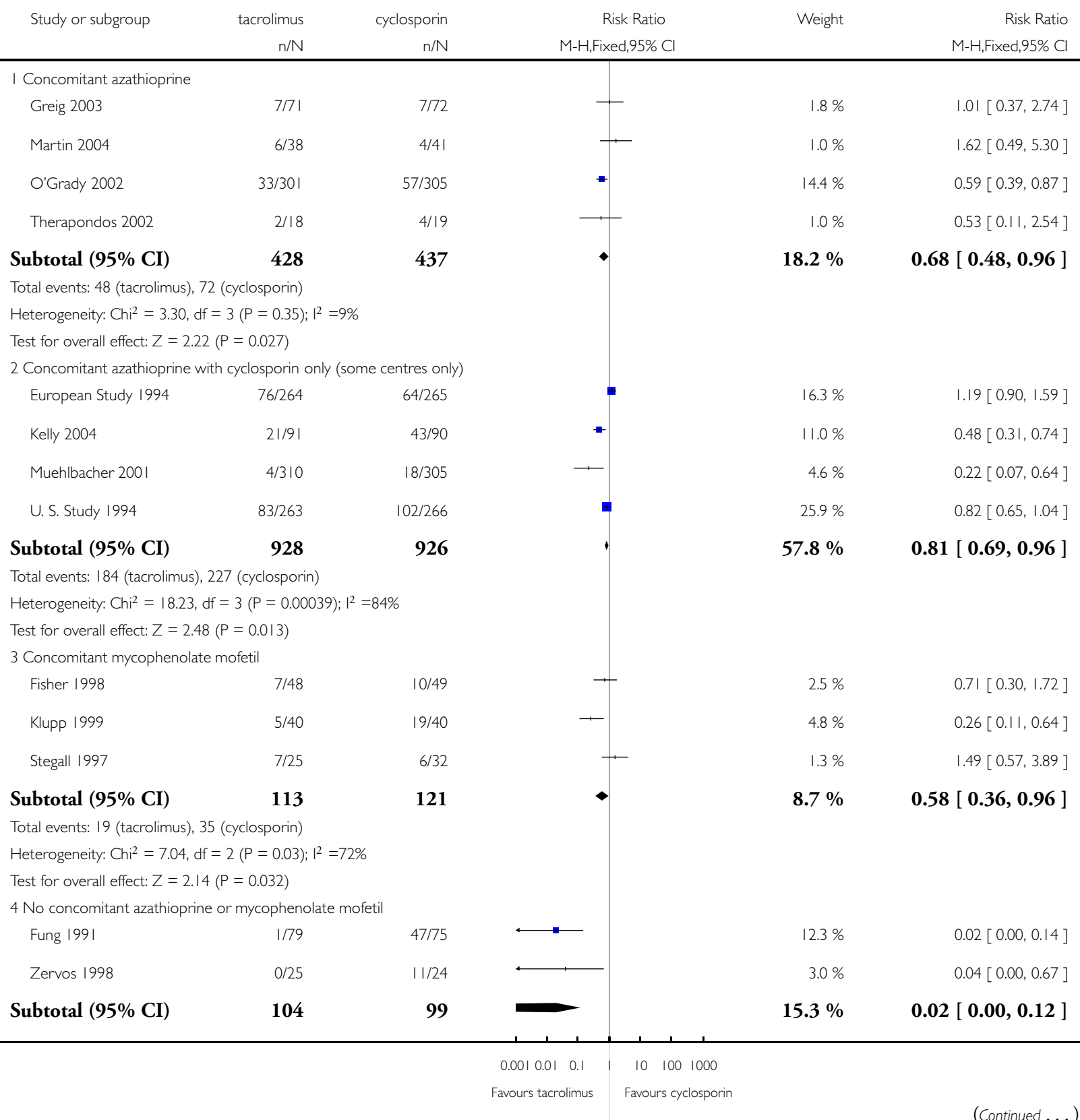




\begin{tabular}{|c|c|c|c|c|c|}
\hline Study or subgroup & tacrolimus & cyclosporin & $\begin{array}{c}\text { Risk Ratio } \\
\text { M-H,Fixed,95\% Cl }\end{array}$ & Weight & $\begin{array}{r}\text { (... Continued) } \\
\text { Risk Ratio } \\
\text { M-H Fixed } 95 \% \text { C }\end{array}$ \\
\hline \multicolumn{6}{|c|}{ Total events: I (tacrolimus), 58 (cyclosporin) } \\
\hline \multicolumn{6}{|c|}{ Heterogeneity: $\mathrm{Chi}^{2}=0.18, \mathrm{df}=\mid(P=0.67) ; \mathrm{I}^{2}=0.0 \%$} \\
\hline \multicolumn{6}{|c|}{ Test for overall effect: $Z=4.56(P<0.0000 \mathrm{I})$} \\
\hline Total $(95 \%$ CI $)$ & 1573 & 1583 & $\cdot$ & $100.0 \%$ & $0.65[0.57,0.74]$ \\
\hline \multicolumn{6}{|c|}{ Total events: 252 (tacrolimus), 392 (cyclosporin) } \\
\hline \multicolumn{6}{|c|}{ Heterogeneity: $\mathrm{Chi}^{2}=53.32, \mathrm{df}=12(\mathrm{P}<0.0000 \mathrm{I}) ; \mathrm{I}^{2}=77 \%$} \\
\hline \multicolumn{6}{|c|}{ Test for overall effect: $Z=6.14(P<0.0000 I)$} \\
\hline
\end{tabular}

\section{A P PENDICES}

\section{Appendix I. Search Strategies}

\begin{tabular}{|c|c|c|}
\hline Database & Timespan of search & Search strategy \\
\hline $\begin{array}{l}\text { The Cochrane Hepato-Biliary Group Con- } \\
\text { trolled Trials Register }\end{array}$ & August 2005. & $\begin{array}{l}\text { (c*closporin* OR neoral* OR sandimmun*)AND (tacrolimus } \\
\text { OR prograf OR FK506) AND ('liver transplant*') }\end{array}$ \\
\hline $\begin{array}{l}\text { Cochrane Centrale Register of Controlled } \\
\text { Trials in The Cochrane Library }\end{array}$ & Issue 3, 2005. & $\begin{array}{l}\text { \#1 MeSH descriptor Cyclosporine explode all trees in MeSH } \\
\text { products } \\
\text { \#2 c*closporin* OR neoral* OR sandimmun* in All Fields in all } \\
\text { products } \\
\text { \#3 (\#1 OR \#2) } \\
\text { \#4 MeSH descriptor Tacrolimus explode all trees in MeSH prod- } \\
\text { ucts } \\
\text { \#5 tacrolimus OR prografOR FK506 in All Fields in all products } \\
\text { \#6 (\#4 OR \#5) } \\
\text { \#7 MeSH descriptor Liver Transplantation explode all trees in } \\
\text { MeSH products } \\
\text { \#8 liver transplant* in All Fields in all products } \\
\text { \#9 (\#7 OR \#8) } \\
\text { \#10 (\#3 AND \#6 AND \#9) }\end{array}$ \\
\hline MEDLINE (WinSPIRS 5.0) & 1950 to Aug 2005. & $\begin{array}{l}\text { \#1 explode "Cyclosporine"/all subheadings } \\
\# 2 \text { c*closporin* or neoral* or sandimmun* } \\
\# 3 \text { \#1 or \#2 } \\
\text { \#4 explode "Tacrolimus"/all subheadings } \\
\# 5 \text { tacrolimus or prograf or FK506 } \\
\text { \#6 \#4 or \#5 } \\
\text { \#7 explode "Liver-Transplantation"/all subheadings }\end{array}$ \\
\hline
\end{tabular}




\begin{tabular}{|c|c|c|}
\hline & & $\begin{array}{l}\# 8 \text { liver transplant* } \\
\# 9 \# 7 \text { or } \# 8 \\
\# 10 \# 3 \text { and } \# 6 \text { and } \# 9 \\
\# 11 \text { random* or placebo* or blind }{ }^{*} \text { or meta-analysis } \\
\# 12 \# 10 \text { and } \# 11\end{array}$ \\
\hline EMBASE (WinSPIRS 5.0) & 1980 to Aug 2005. & $\begin{array}{l}\text { \#1 explode "cyclosporin"/all subheadings } \\
\# 2 \text { c closporin* or neoral* or sandimmun* } \\
\# 3 \text { \#1 or \#2 } \\
\# 4 \text { explode "tsukubaenolide"/all subheadings } \\
\# 5 \text { tacrolimus or prograf or FK506 or tsukubaenolide } \\
\# 6 \text { \#4 or \#5 } \\
\# 7 \text { explode "liver-transplantation"/all subheadings } \\
\# 8 \text { liver transplant* } \\
\# 9 \text { \#7 or \#8 } \\
\# 10 \text { \#3 and \#6 and \#9 } \\
\# 11 \text { random* or placebo* or blind* or meta-analysis } \\
\# 12 \text { \#10 and \#11 }\end{array}$ \\
\hline $\begin{array}{l}\text { Science Citation Index Expanded (http:// } \\
\text { portal.isiknowledge.com/portal.cgi? } \\
\text { DestApp=WOS\&Func=Frame) }\end{array}$ & 1945 to Aug 2005. & $\begin{array}{l}\text { \#1 TS=(c*closporin* OR neoral* OR sandimmun*) } \\
\text { \#2 TS=(tacrolimus OR prograf OR FK506) } \\
\text { \#3 TS=(liver transplant*) } \\
\text { \#4 \#3 AND \#2 AND \#1 } \\
\text { \#5 TS=(random* or placebo* or blind* or meta-analysis) } \\
\text { \#6 \#5 AND \#4 }\end{array}$ \\
\hline
\end{tabular}

\section{WHAT'S NEW}

Last assessed as up-to-date: 23 August 2006.

17 October 2008 Amended Converted to new review format.

\section{H I S T O R Y}

Protocol first published: Issue 1, 2005

Review first published: Issue 4, 2006 


\section{CONTRIBUTIONS OF AUTHORS}

All authors contributed to the design of the protocol. V McAlister and E Haddad evaluated whether the trials fulfilled the inclusion criteria. E Renouf hand searched reference sections of the reports for other randomised clinical trails, and prepared blinded copies of all selected reports. V McAlister and E Haddad independently extracted data. R Malthaner adjudicated differences. All authors reviewed trial selection and data extraction. V McAlister wrote to investigators and to the sponsoring companies of included trials to ask for data that are not presented in the published reports. V McAlister and E Haddad performed all meta-analyses in RevMan. LL Gluud and M Kjaer performed additional blinded statistical analyses including meta-regression and regression analyses of funnel plot asymmetry. All authors contributed to and approved the review.

\section{DECLARATIONS OF INTEREST}

V McAlister has taken part in clinical trials of tacrolimus and cyclosporin in liver and kidney transplantation. He has received grantsin-aid for laboratory research from Sandoz (Novartis) and Fujisawa (Astelis).

\section{INDEX TERMS \\ Medical Subject Headings (MeSH)}

*Liver Transplantation [mortality]; Cyclosporine [adverse effects; *therapeutic use]; Graft Rejection [mortality; * prevention \& control]; Graft Survival; Immunosuppressive Agents [adverse effects; *therapeutic use]; Randomized Controlled Trials as Topic; Tacrolimus [adverse effects; * therapeutic use]; Time Factors

\section{MeSH check words}

Humans 